\title{
The Tensile Strength of Porous Copper Made by the GASAR Process
}

\author{
by \\ Anthony E. Simone, Jr. \\ Bachelor of Science, Swarthmore College, Swarthmore, PA, 1992 \\ Submitted to the Department of Civil and Environmental Engineering \\ in Partial Fulfillment of the Requirements for the Degree of \\ Master of Science \\ in Civil and Environmental Engineering \\ at the \\ Massachusetts Institute of Technology
}

August 1994

(C) 1994 Massachusetts Institute of Technology

All rights reserved

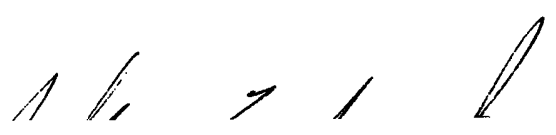

Signature of the Author

Departhent of Civil and Environghental Engineering
August 12, 1994

Certified by

Professor Lorna J. Gibson

Thesis Supervisor

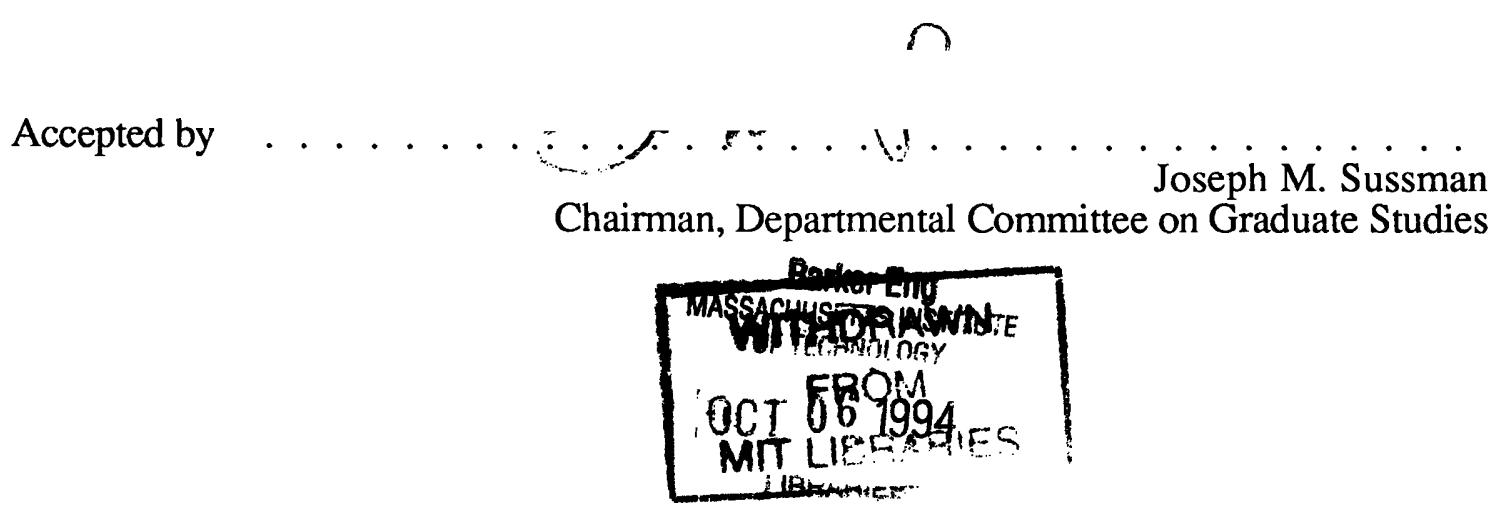




\title{
The Tensile Strength of Porous Copper Made by the GASAR Process
}

\author{
by \\ Anthony E. Simone, Jr. \\ Submitted to the Department of Civil and Environmental Engineering \\ in Partial Fulfillment of the Requirements for the Degree of \\ Master of Science in Civil and Environmental Engineering
}

\begin{abstract}
The GASAR process is a method of producing anisotropic porous materials through a directional solid-gas eutectic solidification. Elongated cylindrical pores form in the direction of solidification. An experimental study was carried out on cylindrical ingots of copper-based GASAR materials with axially oriented cylindrical pores. Materials with porosities ranging from 0.14 to 0.50 and nominal pore sizes of $10 \mu \mathrm{m}, 50 \mu \mathrm{m}$ and 500 $\mu \mathrm{m}$ were obtained. Directionally solidified nonporous copper ingots were also obtained. Tensile specimens with a reduced diameter of $5 \mathrm{~mm}$ were machined from the GASAR ingots. All specimens were uniaxially tested with the cylindrical pores oriented in the loading direction. The $0.2 \%$ yield strength, the ultimate tensile strength, the Young's modulus, and the ductility (strain at peak stress) were measured from the uniaxial stressstrain curve. These values were normalized with respect to the net solid area of the specimen and plotted as a function of porosity.
\end{abstract}

A characterization of the microstructure of the porous and nonporous specimens was also performed. The grain size was measured in the transverse and longitudinal directions using the linear intercept method. The average pore dimensions and pore size distribution were also measured. Other measurements and observations were made in order to characterize the relationship between the pores and grains with respect to porosity. The crystallographic texture and impurity content of both porous and nonporous specimens were measured.

The net area yield strength of the porous specimens was consistently higher than the measured yield strength of the nonporous copper. The net area ultimate tensile strength decreased with increasing porosity in the porous GASAR specimens, but specimens with a porosity below 0.23 had a higher net area ultimate tensile strength than the nonporous copper. The net area Young's modulus was independent of porosity. The ductility decreased significantly with increasing porosity. All materials were found to contain columnar grains oriented in the direction of testing. Both the grains and the pores were found to have an aspect ratio of approximately ten. The grains of the nonporous material were much larger than those of the porous samples. All materials were found to have a strong $<100>$ crystallographic texture in the loading direction. Both the nonporous and porous materials were found to be approximately $99.98 \%$ pure copper. The possible influence of the measured microstructural characteristics on the higher net area yield strength of the porous specimens is discussed. Several effects are presented and discussed which may explain the increase.

Thesis Supervisor - Lorna J. Gibson

Title - Associate Professor of Civil and Environmental Engineering 


\section{Acknowledgments}

First of all, I would like to thank Professor Loma Jane Gibson for her guidance and support in the completion of this thesis. Her diligence and attention to detail have provided an excellent example to me and others under her supervision. She has taught me many things which can not be learned in a classroom.

I would like to thank the Office of Naval Research for their financial support, which made this research possible. I would also like to thank Dr. Amitov Pattnaik and Dr. Virgil Provenzano of the Naval Research Laboratories for their input and support.

I would like to thank Mr. Steve Rudolph and Mr. Arthur Rudolph of the Civil Engineering machine shop, and Mr. Ken Stone of the MIT Hobby Shop. Their craftsmanship and enthusiastic advice has been invaluable to the completion of this thesis.

To Valeria Lacayo, I offer my deepest appreciation for her unwavering support and inexhaustible tolerance.

Finally, I would like to thank my parents, Andrea and Anthony, Sr., and my sisters, Michelle and Nicole. The love and support of my family has been my beacon. 


\section{Table of Contents}

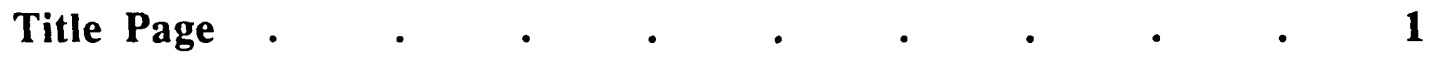

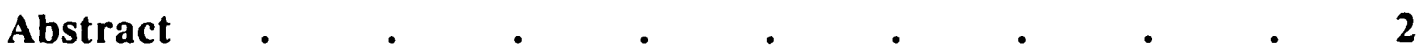

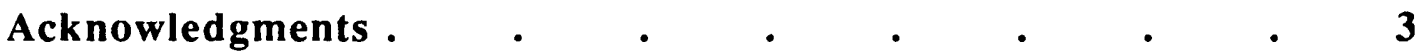

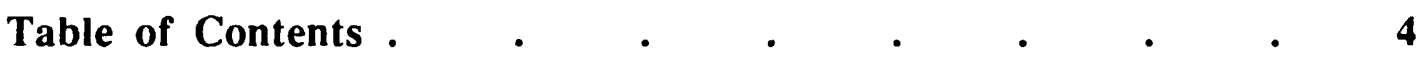

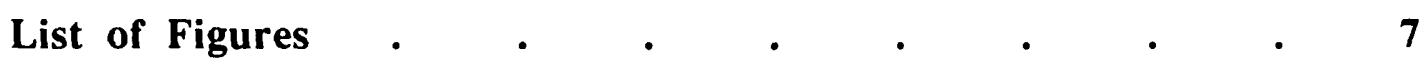

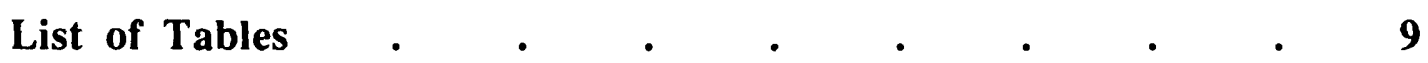

Chapter One : Introduction and Background $\quad$ - 10

1.1 The GASAR Process . . . . . . . . . 10

1.2 Currently Available Base Materials and Microstructures . . . $\quad 14$

1.3 Properties of GASAR Materials . $\quad$. $\quad$. $\quad$. $\quad$. $\quad$.

1.3.1 Tensile Strengths $\quad$. $\quad$. $\quad$. $\quad$. $\quad$. $\quad$. 18

1.3.2 Other Mechanical Properties . . . . . . $\quad$. 23

1.3.3 Thermal Properties . $\quad$. $\quad$. $\quad$. $\quad$. $\quad$. 23

1.4 Applications of GASAR Materials $\quad$. $\quad$. $\quad$. $\quad$. $\quad$. 24

1.4.1 Current Applications $\quad . \quad$. $\quad$. $\quad$. $\quad$. $\quad$. 25

1.4.2 Structural Applications . $\quad$. $\quad$. $\quad$. $\quad$. $\quad 25$

1.5 The Gibson and Ashby Models for Cellular Solids . . . $\quad$. 27

1.5.1 Out-of-Plane Behavior of Honeycombs $\quad$. $\quad$. $\quad 27$

1.5.2 Open-Cell Foamed Materials $\quad$. $\quad$. $\quad$. $\quad$. $\quad 29$

1.5.3 Closed-Cell Foamed Materials . $\quad$. $\quad$. $\quad$. $\quad 32$

1.5.4 Anisotropic Unit Cells . $\quad$. $\quad$. $\quad$. 32

1.6 Comparison of Gibson and Ashby Models to GASAR Behavior a 33

1.7 Related Topics . . . . . . . . . . 34

1.7.1 FCC Crystal Structure and Slip Systems . . . $\quad 35$

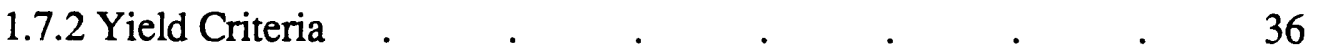

1.7.3 Unidirectional Solidification . $\quad . \quad$. $\quad . \quad$. 38

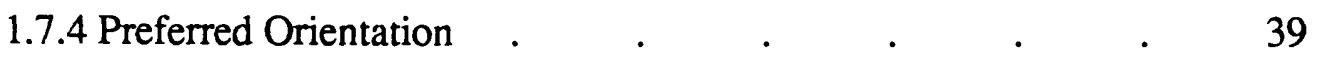

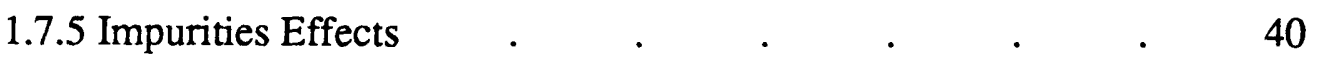

1.7.6 The Hall-Petch Effect . . $\quad . \quad$. $\quad$. $\quad$. $\quad$. 41

1.7.7 Specimen Size Effects. . $\quad$. $\quad$. $\quad$. $\quad$. 42

1.5 Thesis Goals $\quad . \quad$. $\quad$. $\quad$. $\quad$. $\quad$. $\quad$. $\quad$. 43 
Chapter Two: Experimental Methods . . . . 44

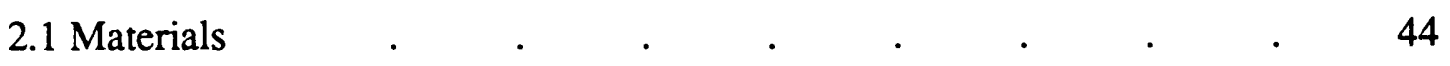

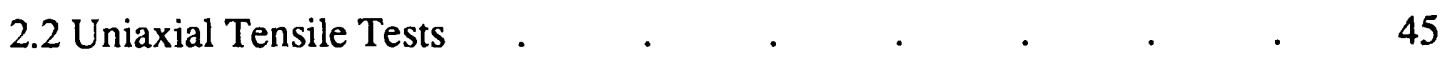

2.2.1 Specimen Design $\quad . \quad$. $\quad . \quad$. $\quad . \quad$. $\quad . \quad$. 45

2.2.2 Testing Equipment and Procedure . . . . . $\quad$. 46

2.3 Microscopy Methodology $\quad$. $\quad$. $\quad$. $\quad$. $\quad$. $\quad 52$

2.3.1 Polishing and Etching Procedures $\quad$. $\quad$. $\quad$. $\quad$. 53

2.3.2 Microscopic Examination $\quad . \quad$. $\quad$. $\quad$. $\quad$. 54

2.3.3 Grain and Pore Size Measurements . $\quad$. $\quad$. 56

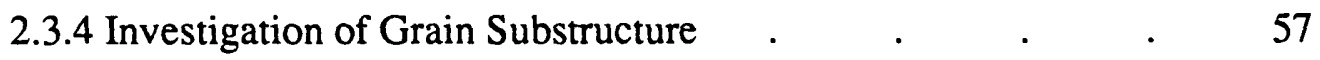

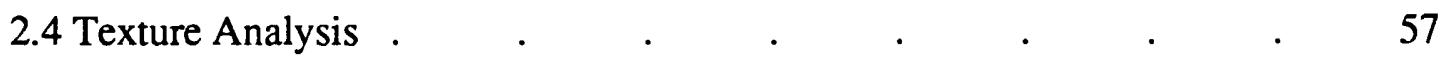

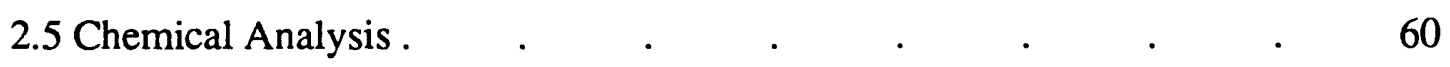

Chapter Three : Presentation of Results - • $\quad 62$

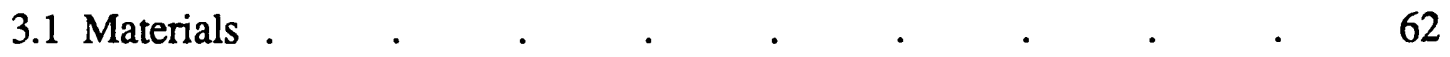

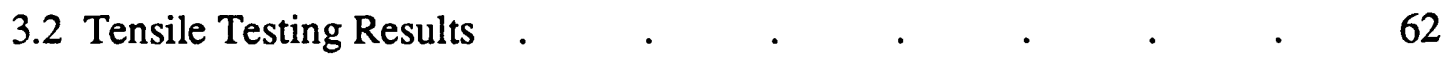

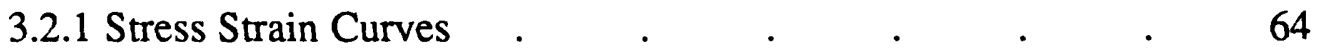

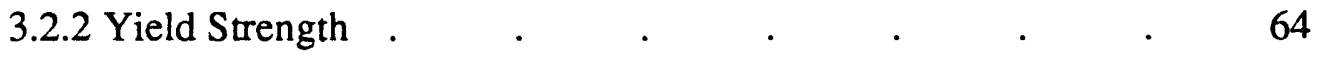

3.2.3 Ultimate Tensile Strength $\quad . \quad$. $\quad$. $\quad$. $\quad$. 71

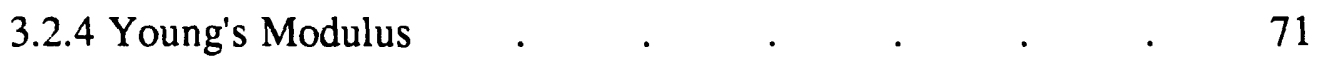

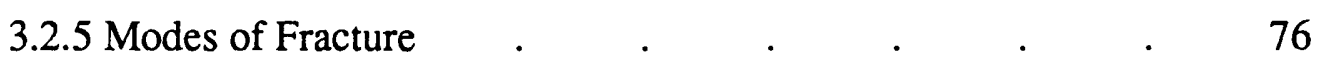

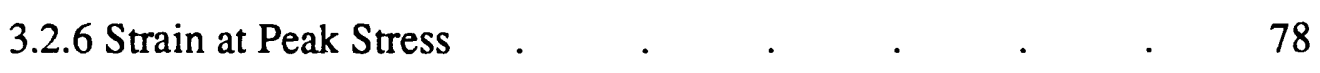

3.3 Cross-Sectional Micrographs $\quad$. $\quad$. $\quad$. $\quad$. $\quad$. $\quad$. 78

3.4 Fracture Surface Micrographs . $\quad$. $\quad$. $\quad$. $\quad$. $\quad$. 79

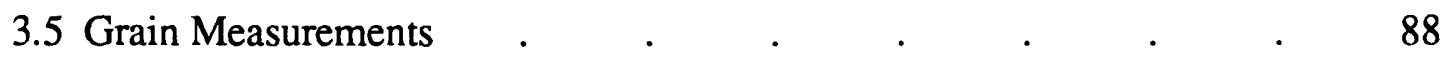

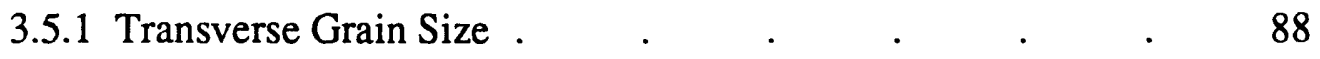

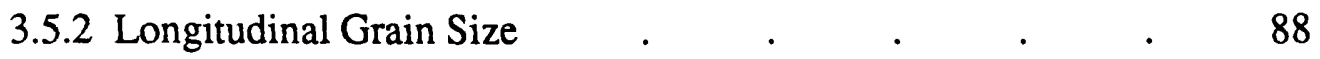

3.6 Microstructural Characterization . $\quad$. $\quad$. $\quad$. $\quad$. $\quad$. 91

3.6.1 Relationship Between Pore and Grain Structure . . 91

3.6.2 Characterization of the Grain Boundary . $\quad$. $\quad$. 92

3.6.3 The Measured Pore Size and Size Distribution . $\quad 92$

3.6.4 Characterization Summary . . . . . . . $\quad$. 95

3.7 Hall-Petch Data . $\quad$. $\quad$. $\quad$. $\quad$. $\quad$. $\quad$. 95 


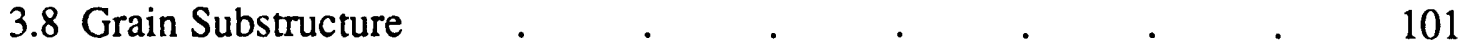

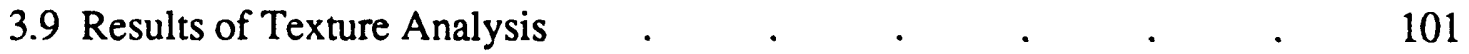

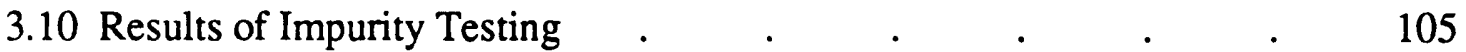

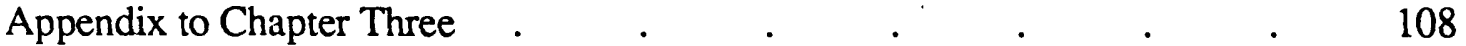

Chapter Four : Discussion of Results . . . . 110

4.1 discussion of Tensile Testing Results . $\quad$. $\quad$. $\quad$. $\quad$. 110

4.2 Effect of Grain Size vs. Specimen Size . $\quad$. $\quad$. $\quad$. $\quad$. 112

4.3 The Hall-Petch Effect $\quad$. $\quad$. $\quad$. $\quad$. $\quad$. 112

4.3.1 Influence of the Porous Grain Boundary on the Hall-Petch Effect 113

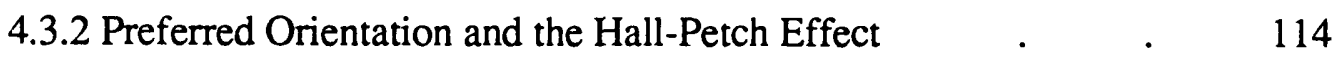

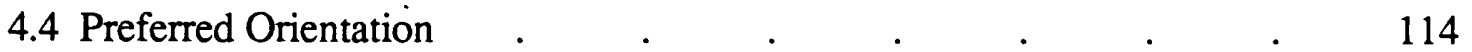

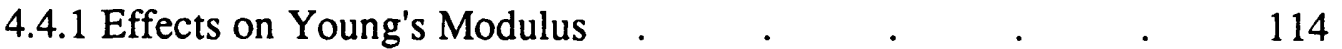

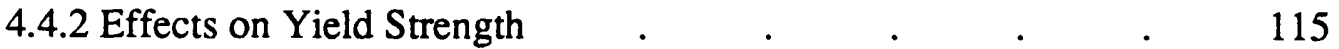

4.5 Constraining Effects of the Porous Microstructure $\quad$. $\quad$. $\quad 117$

4.5.1 Triaxial and Plane Stress States $\quad . \quad$. $\quad . \quad$. 118

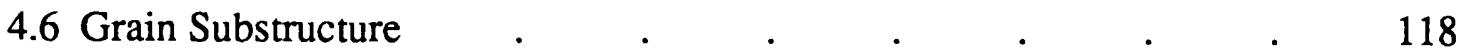

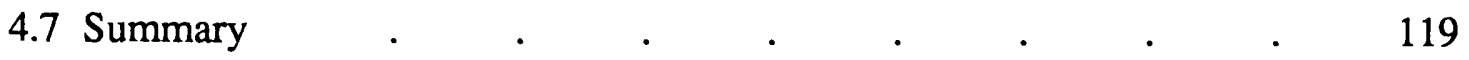

Chapter Five: Conclusions $\quad$ - . . . . . 120

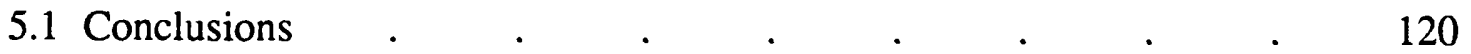

5.2 Recommendations for Future Research . $\quad$. $\quad$. $\quad$. $\quad$. 121

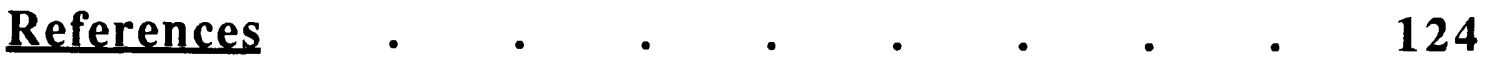

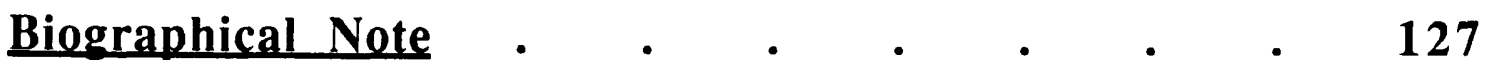




\section{List of Figures}

Figure 1.1 - Representative Metal-Hydrogen Phase Diagram $\quad$. $\quad$. 11

Figure 1.2 - Schematic Diagram of Laboratory Apparatus for Casting GASAR

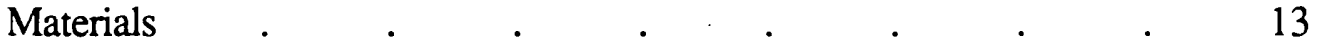

Figure 1.3 - Material Availability vs. Pore Size and Porosity $\quad$ - $\quad 16$

Figure 1.4 - Potential Pore Morphology and Architecture $\quad$ • $\quad$. $\quad$. 17

Figure 1.5 - DMI Yield Strength vs. Porosity Data . $\quad$. $\quad . \quad$. 19

Figure 1.6 - DMI Ultimate Tensile Strength vs. Porosity Data $\quad$. $\quad 20$

Figure 1.7 - Idealized Honeycomb Cellular Material . $\quad$. $\quad$. $\quad$. 28

Figure 1.8 - Idealized Open-Cell Foam $\quad . \quad$. $\quad$. $\quad$. $\quad$. 30

Figure 1.9 - Idealized Closed-Cell Foam $\quad . \quad$. $\quad$. $\quad . \quad$. 31

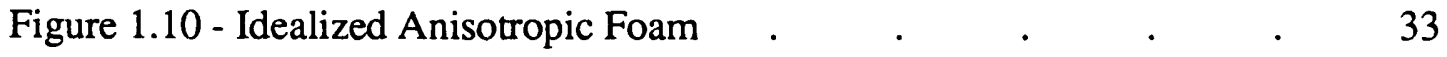

Figure 1.11 - Unit Cell of FCC Metals $\quad . \quad$. $\quad . \quad$. $\quad . \quad$. $\quad 35$

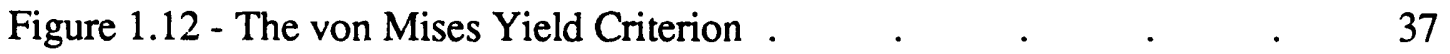

Figure 1.13 - Relationship Between Pore and Grain Size 42

Figure 2.1 - Design of Tensile Specimen (5 mm Diameter) . . . . . 47

Figure 2.2 - Design of Tensile Specimen (12.5 mm Diameter) . $\quad$. 48

Figure 2.3 - Schematic Diagram of Tensile Testing Apparatus $\quad$. $\quad$. 50

Figure 2.4 - Schematic Diagram of Grips for $12.5 \mathrm{~mm}$ Specimens . $\quad$. $\quad 51$

Figure 2.5 - Schematic Diagram of a Scanning Electron Microscope . $\quad 55$

Figure 2.6 - Illustration of Bragg's Law for Diffraction $\quad . \quad$. $\quad . \quad 58$

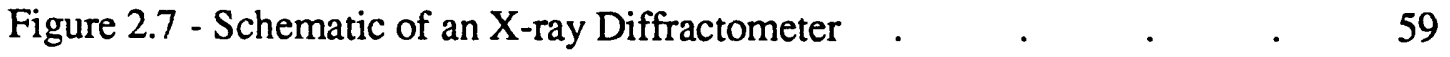

Figure 3.1 - Distribution of GASAR Specimen Porosities 63

Figure 3.2 - Stress-Strain Curves of Nonporous Specimens . . . 65

Figure 3.3 - Stress-Strain Curves of $10 \mu \mathrm{m}$ Pore Size Specimens $\quad 66$

Figure 3.4 - Stress-Strain Curves of $50 \mu \mathrm{m}$ Pore Size Specimens . . 67

Figure 3.5 - Stress-Strain Curves of $500 \mu \mathrm{m}$ Pore Size Specimens . $\quad$. $\quad 68$

Figure $3.6-0.2 \%$ Yield Strength vs. Porosity $\quad . \quad$. $\quad . \quad$. $\quad$. 69

Figure 3.7 - Net Area 0.2\% Yield Strength vs. Porosity . . . . 70

Figure 3.8 - Ultimate Tensile Strength vs. Porosity . • . . $\quad$ • 72

Figure 3.9 - Net Area Ultimate Tensile Strength vs. Porosity . 73

$\begin{array}{lllllllll}\text { Figure } 3.10 \text { - Young's Modulus vs. Porosity } \quad . \quad & \text { • } & & 74\end{array}$

Figure 3.11 - Net Area Young's Modulus vs. Porosity $\quad . \quad$. $\quad$. 75 
Figure 3.12 - Variation in Modes of Fracture . . . . . $\quad 76$

Figure 3.13 - Stain at Peak Stress vs. Porosity d . $\quad$. $\quad$. 77

Figure 3.14 - Transverse Cross-Section Micrographs of $10 \mu \mathrm{m}$ Nominal

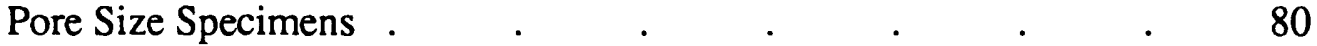

Figure 3.15 - Transverse Cross-Section Micrographs of $50 \mu \mathrm{m}$ Nominal

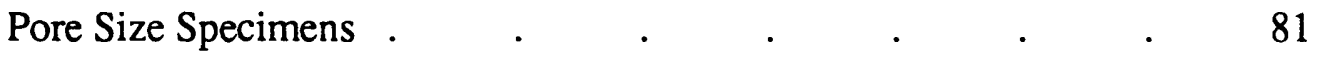

Figure 3.16 - Transverse Cross-Section Micrographs of $500 \mu \mathrm{m}$ Nominal

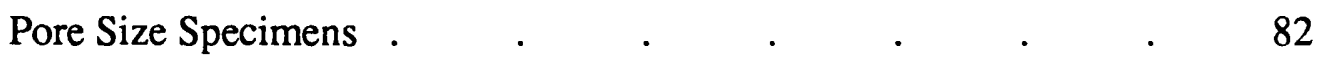

Figure 3.17 - Longitudinal Cross-Section Micrographs of $10 \mu \mathrm{m}$ Nominal

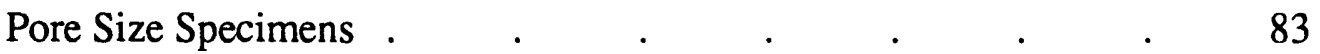

Figure 3.18 - Longitudinal Cross-Section Micrographs of $50 \mu \mathrm{m}$ Nominal

Pore Size Specimens . $\quad$. $\quad$. $\quad$. $\quad$. $\quad$. $\quad$. 84

Figure 3.19 - Longitudinal Cross-Section Micrographs of $500 \mu \mathrm{m}$ Nominal

Pore Size Specimens . $\quad$. $\quad$. $\quad$. $\quad$. 85

Figure 3.20 - Fracture Surface Micrographs . $\quad$. $\quad$. $\quad$. $\quad 36$

Figure 3.21 - Transverse Grain Size vs. Porosity $\quad$. $\quad$. $\quad$. $\quad$. $\quad 89$

Figure 3.22 - Longitudinal Grain Size vs. Porosity . . . . $\quad$. 90

Figure 3.23 - Percentage of Grain Boundary Consisting of Solid Interface

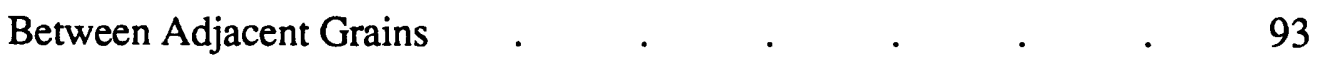

Figure 3.24 - Microstructural Characterization Summary for Specimens with

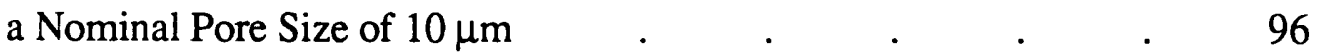

Figure 3.25 - Microstructural Characterization Summary for Specimens with

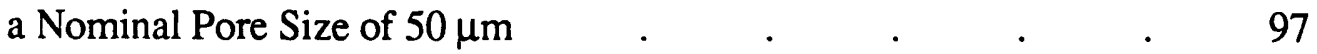

Figure 3.26 - Microstructural Characterization Summary for Specimens with

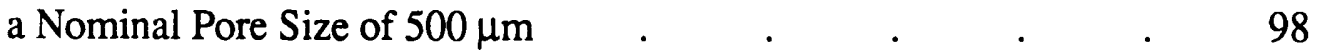

Figure 3.27 - Hall-Petch Data for GASAR Specimens $\quad$. $\quad$. $\quad$. 99

Figure 3.28 - Comparison of GASAR Data with Hall-Petch Data From Other

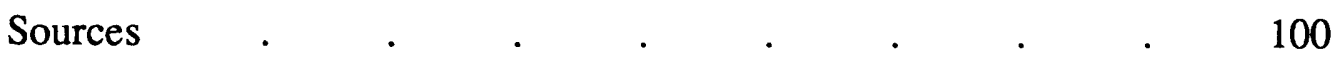

Figure 3.29- Grain Substructure Micrographs _ . $\quad$. $\quad$. $\quad . \quad 102$

Figure 3.30 - Sample Diffractometer Test Results . . . . . 103

Figure 3.31 - Sample Electron Probe Results . . . . . $\quad 106$

Figure 4.1 - Polycrystalline Yield Strength vs. Texture $\quad$. $\quad$. $\quad$. 116

Figure 4.2 - Areas of Plane Stress and Triaxial Stress States . $\quad$. $\quad 118$ 


\section{List of Tables}

Table 1.1 - Base Materials, Pore Sizes, and Porosities Possible with GASAR

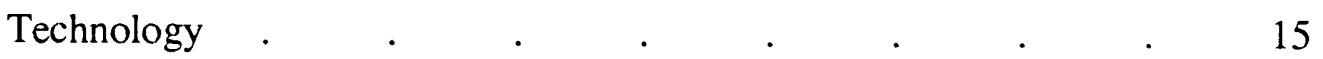

Table 1.2 - Mechanical Wear Tests on GASAR Materials . . . . $\quad 23$

Table 3.1 - Measured Transverse Pore Size . . . . . . $\quad$. 94

Table 3.2 - Results of Impurity Analysis $\quad . \quad$. $\quad$. $\quad$. $\quad$. 107

Table 3.A.1 - Tensile Test Data . . . . . . . . . . 108

Table 3.A.2 - Microscopy Data $\quad . \quad$. $\quad . \quad$. $\quad . \quad . \quad 109$ 


\section{Chapter One : Introduction and Background}

The GASAR process is an innovative method of producing isotropic or highly anisotropic porous materials. The method was developed by Dr. V.I. Shapovalov at the Dnepropetrovsk Metallurgical Institute (DMI) in Ukraine. It is a new process, the U.S. patent being issued in January of 1993. GASAR technology is based on a solid-gas eutectic solidification process in which pore nucleation results from the diffusion of gaseous hydrogen out of a liquid melt as it freezes. The volume fraction of pores, or porosity, of the solidified material is determined by the chamber pressure of the hydrogen atmosphere at which the process takes place. The size, shape and orientation of the pores can also be controlled through variation of the cooling rate and cooling direction. Compared to other production methods of porous materials, the GASAR process is quick, requiring as little as 10 minutes to produce $30 \mathrm{~kg}$ of material at its present level of development ${ }^{1}$. The following chapter describes the GASAR process, the microstructures available, the mechanical and thermal properties and the potential applications.

\subsection{The GASAR Process}

In the GASAR process, a liquid metal containing dissolved hydrogen gas is cooled through the eutectic point. This cooling process is depicted on a representative isobaric metal-hydrogen phase diagram in Figure 1.1. As the eutectic point is reached, the nucleation of pores, due to gas diffusion, and the solidification of the liquid metal occur simultaneously. The diffusion of hydrogen is driven by the large decrease in hydrogen solubility that occurs with the transition to crystalline form ${ }^{2}$. The volume of the gaseous hydrogen phase in the resulting material depends on the weight percent of hydrogen at 


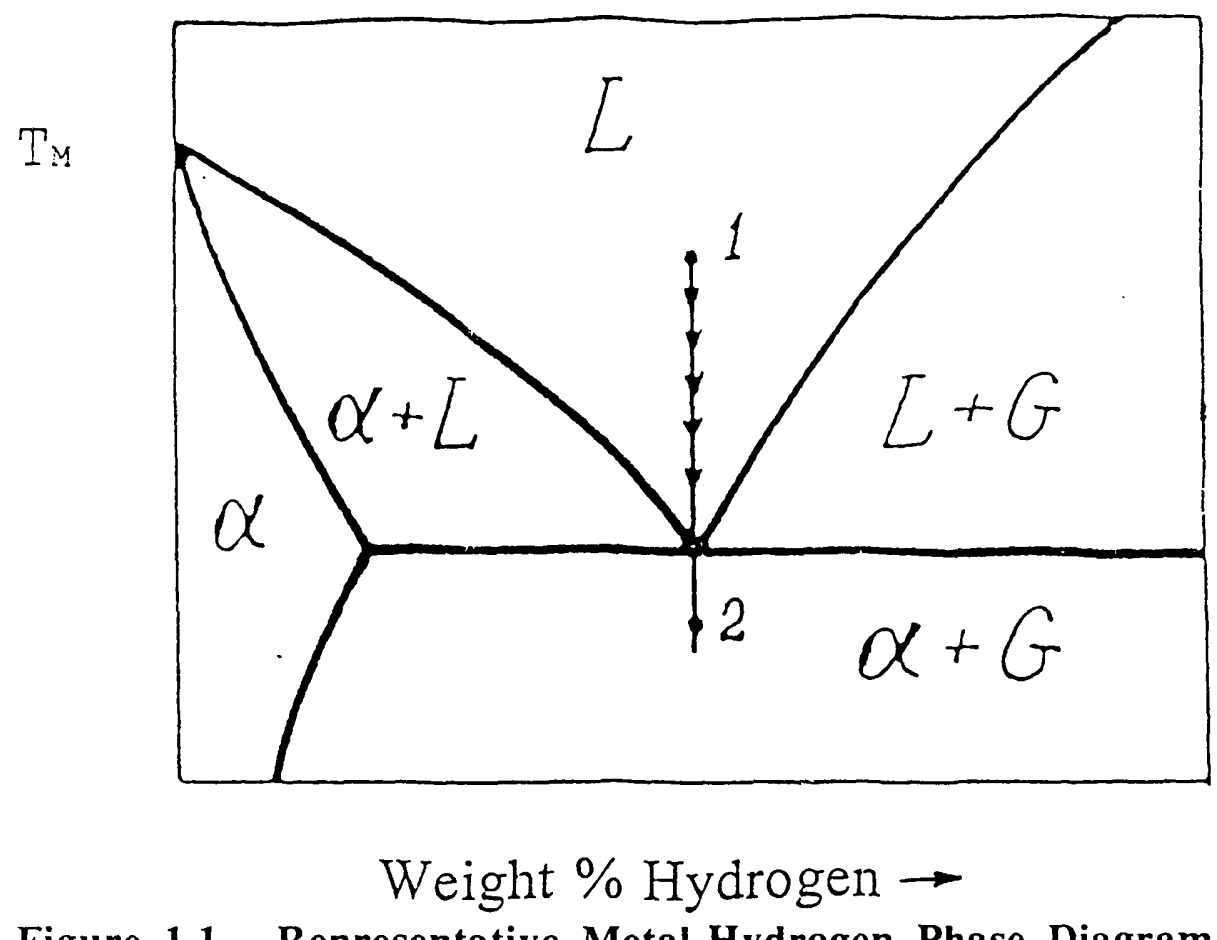

Figure 1.1 - Representative Metal-Hydrogen Phase Diagram

The cooling path 1-2 indicates the cooling process involved in the casting of GASAR materials. At point 1 , the liquid metal is saturated with dissolved hydrogen. At point 2, the hydrogen has diffused out of the metal to form pores as the metal freezes. (Ref. 1)

which the eutectic point exists. Since the position of the eutectic point depends on the pressure, the porosity of GASAR materials can be precisely controlled by manipulation of the pressure of the hydrogen atmosphere in the casting chamber.

Because temperature has a significant effect on the solubility of hydrogen within a liquid metal ${ }^{2}$, the temperature of the melt before the cooling process occurs must also be controlled. This temperature must be coordinated with the hydrogen pressure in order to match the solubility of hydrogen in the melt with the weight percent hydrogen of the eutectic point. If the temperature and pressure are not properly coordinated, cooling will not pass through the eutectic point and a pro-eutectic phase will be formed. This would correspond to a shift to the left or right of the cooling path shown in Figure 1.1. If the hydrogen dissolved in the melt is less than the eutectic weight percentage, a portion of the 
melt will solidify before pore nucleation begins. If the eutectic percentage is exceeded, diffusion of hydrogen will occur before solidification begins, and bubbles will form in the liquid melt. Either of these conditions would result in a lack of consistency in the microstructure of the solidified material.

In addition to the porosity, the size, shape and orientation of the pores can also be controlled. In the GASAR process, pores nucleate at the solidification front, which is the boundary between the solidified material and the liquid melt. Because diffusion is a time dependent process, the movement rate of the solidification front directly influences the distance over which hydrogen can diffuse through the liquid melt before it solidifies. As the amount of diffusion decreases, the size of the nucleated pores will also decrease, as more nucleation sites become necessary. Therefore, the cooling rate used in the GASAR process can be controlled in order to influence the pore size of the solidified material.

As the solidification front moves into the liquid melt, hydrogen tends to diffuse to the pores that have already nucleated on the front. The result of this is that the pores in the solidified material are greatly elongated in the direction of solidification front movement. Because the solidification front moves in the direction of greatest temperature gradient, the orientation of the elongated pores can be controlled by the cooling direction. Unidirectional cooling from the bottom plate of the mold results in longitudinal cylindrical pores, while cooling from the sides of a cylindrical mold results in radial pores. It has been reported by DMI that spherical pores can be produced by pulsing the pressure in the casting chamber during unidirectional cooling.

The apparatus used to make GASAR materials is shown schematically in Figure 1.2. The melt is subjected to a hydrogen atmosphere at a controlled pressure and temperature to allow hydrogen diffusion into the liquid. It is then poured into a chamber where a heat sink is configured to cool the melt at the desired rate and to maintain a directional cooling gradient in order to achieve the desired pore structure in the solidified 


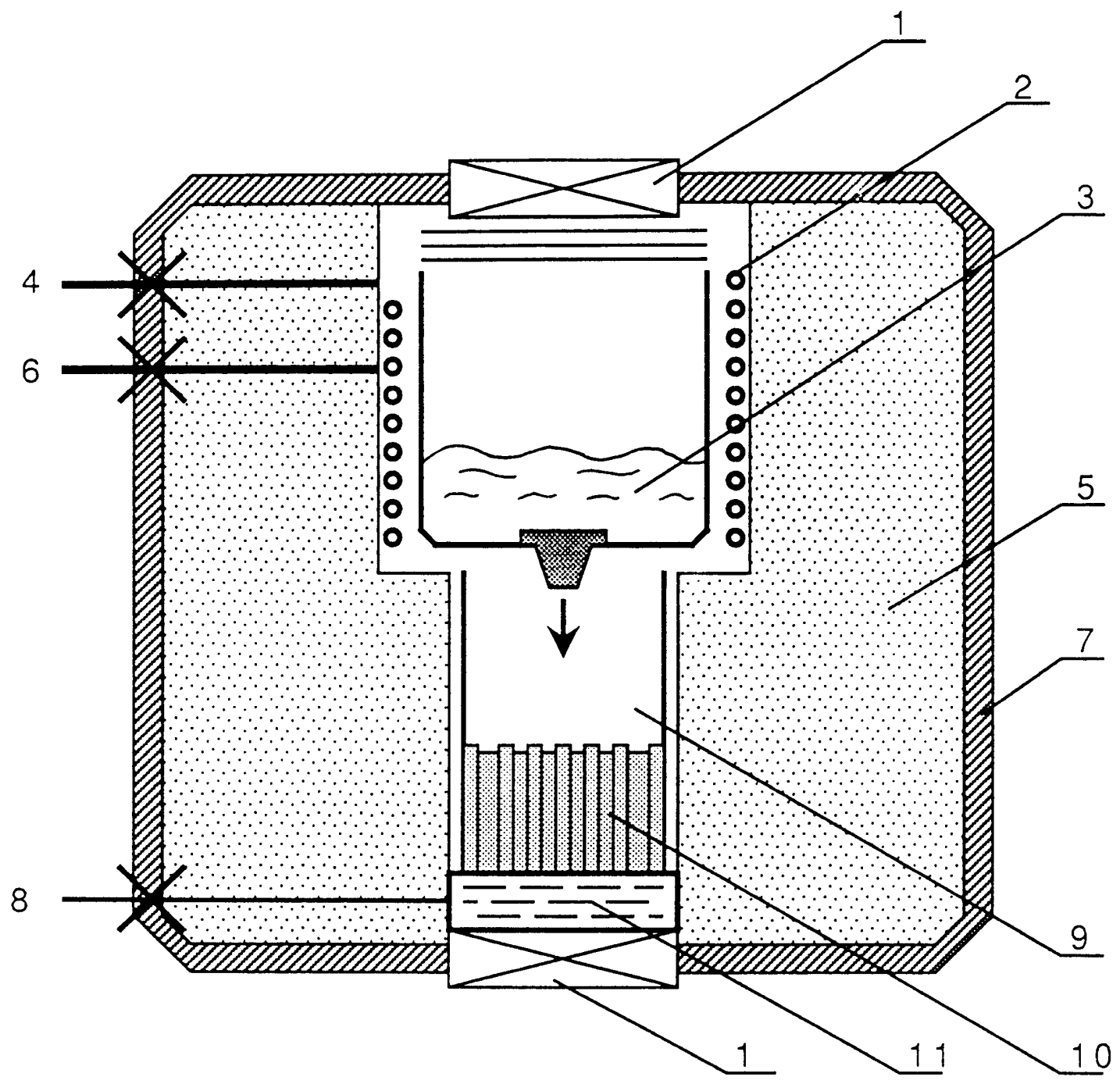

1- TOP AND BOTTOM COVERS

2- HEATING ELEMENTS

3- LIQUID MELT

4- GAS SUPPLY AND EVACUATION SYSTEM

5- HEAT SHIELDING MEANS

6- HEAT SUPPLY SYSTEM

7- HERMETICALLY CAST SHELL

8- MOLD COOLING SYSTEM

9- SOLIDIFYING MELT

10- SOLIDIFIED GASAR WITH AXIAL PORES

11- HEAT SINK FOR DIRECTIONAL SOLIDIFICATION

Figure 1.2 - Schematic Diagram of the Laboratory

Apparatus for Casting of GASAR Materials (Ref. 1) 
material. The apparatus shown in Figure 1.2 is configured to produce a material with longitudinally oriented pores.

Many elements of the solid-gas eutectic solidification used in the GASAR process are not completely understood and are being researched at the present time. At the core of this research are the possible differences between the formation of solid-gas eutectic material and the formation behavior of eutectic materials with two solid phases, such as pearlitic steel. It is hoped that the level of engineering which has been developed with other types of eutectic materials can also be developed with GASAR materials.

\subsection{Currently Available Base Materials and Microstructures}

The base materials, pore sizes and porosities available at the current level of GASAR technology development are summarized in Table 1.1. Note that this list includes metals which are now common in a variety of structural and mechanical applications. None of the metals used with the GASAR process form stable hydrides under normal conditions. The application of the GASAR process to ceramic materials is a recent development. Differences between GASAR materials with ceramic base materials and those with metal base materials have not been fully investigated.

The available pore sizes range from $5 \mu \mathrm{m}$ to $10 \mathrm{~mm}$ and the porosity range is from $5-75 \%$ porous. Because of limitations of hydrogen solubility in some metals ${ }^{2}$, the full range of porosities is not available in certain base materials. The time dependence of diffusion also limits the pore sizes available at a particular porosity for each base metal. The range of possible combinations of pore size and porosity is shown for selected base metals in Figure 1.3. The shaded regions on the graph indicate the ranges for which the process has been used with a high level of success. In general, the pore size must increase with increasing porosity. Copper and magnesium are presently available at the widest range of porosity and pore size combinations. 


\begin{tabular}{|l|l|}
\hline Pure Metals: & $\begin{array}{l}\text { Nickel, Copper, Magnesium, Aluminum, } \\
\text { Molybdenum, Beryllium, Cobalt, } \\
\text { Chromium, Tungsten }\end{array}$ \\
\hline Metal Alloys: & Bronze, Steel, Stainless Steel \\
\hline Ceramics: & Glass, Alumina, Alumina-Magnesia \\
\hline Pore Sizes: & $5 \mu \mathrm{m}-10 \mathrm{~mm}$ \\
\hline Porosities: & $0.05-0.75$ \\
\hline
\end{tabular}

\section{Table 1.1 - Base Materials, Pore Sizes, and Porosities Possible with GASAR Technology (Ref. 1)}

By changing the process control variables (e.g. pressure) during solidification, materials with graded porosity can be produced. By tailoring the porosity gradient to the mechanical requirements of a particular application, a functionally graded material can be produced. Potentially, a material can be produced with alternating levels of solid and porous materials within a monolithic structure. The implications of this possibility are tremendous, since a traditional limiting factor of porous-solid material composites, such as structural sandwich panels, has been the ability to bond the materials effectively. Some of the GASAR material structures with the greatest potential are shown in Figure 1.4. It is claimed by DMI that these material structures can be produced in cylinder or plate form (excluding radial structures) and through the full range of pore size and porosity combinations shown in Figure 1.3. It is also claimed that both open (connected) and closed (unconnected) pore structures can be produced, although this would certainly be highly dependent on the porosity of the material to be formed. Each GASAR pore structure requires a different heat sink configuration to effectively control the temperature 


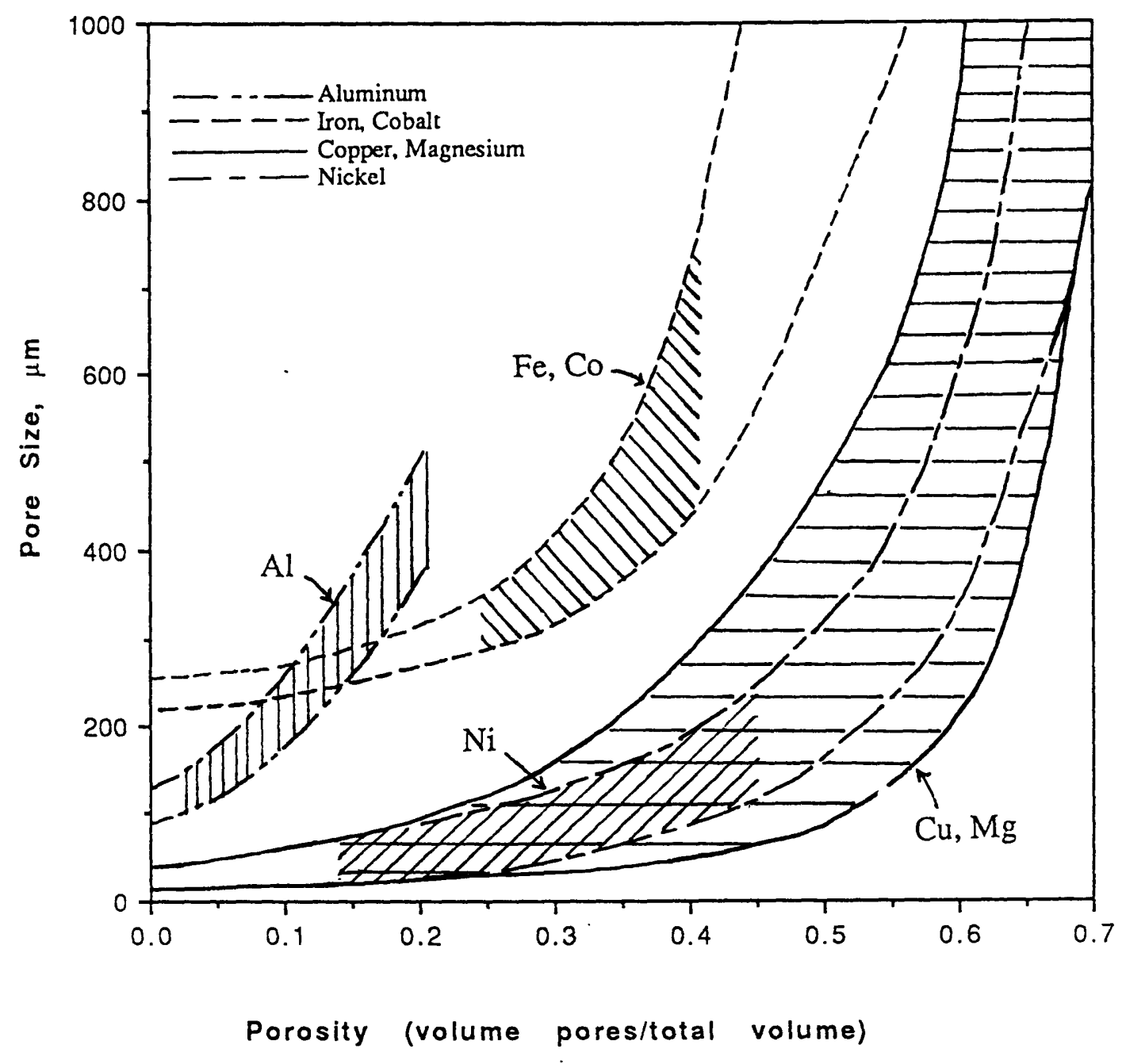

Figure 1.3 - Material Availability vs. Pore Size and Porosity

For several common base materials, the range of possible pore size and porosity combinations are shown. The shaded regions indicate the most stable combinations. The above graph applies for all morphologies. (Ref. 1) 


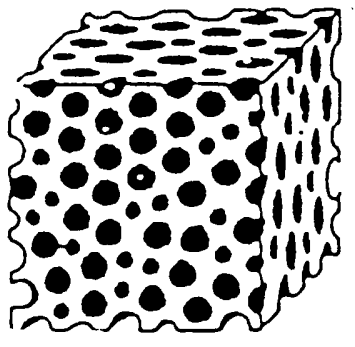

a)

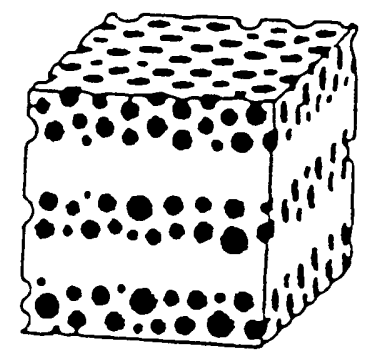

d)

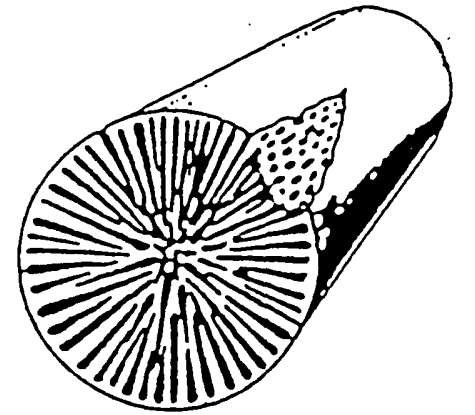

b)

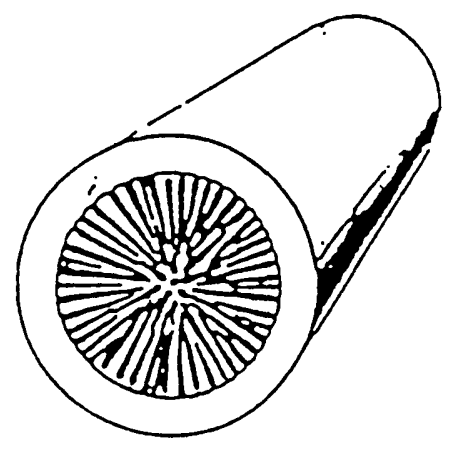

e)

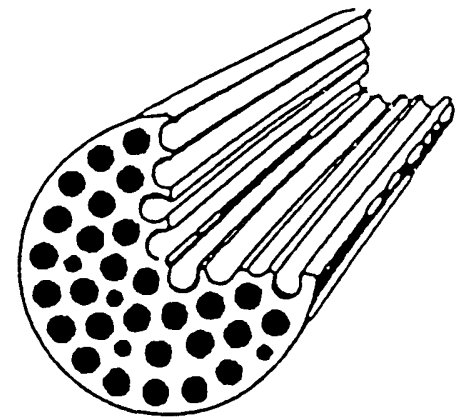

c)

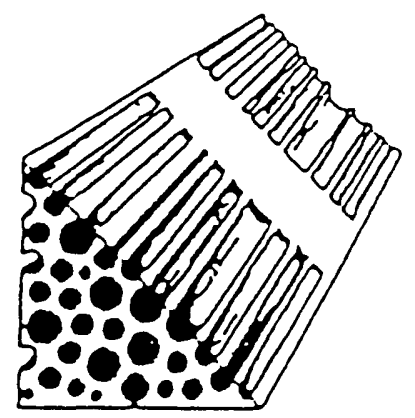

f)

Figure 1.4 - Potential Pore Morphology and Architecture

The above figures represent the possible material forms of GASAR materials as claimed by DMI. They include open or closed pore structures (the above pictures are of closed pores), spherical $(a, d)$ or cylindrical $(b, c, e, f)$ pores, radial $(b, e)$ or longitudinal $(c, f)$ pore orientatons, and layered monolithis structures (d,e,f). (Ref. 1) 
gradient. At the present technology level, the GASAR forms most effectively produced are materials with uniform porosity and longitudinally oriented cylindrical pores. The connectivity of these pore structures with relation to pore size and porosity is undetermined.

\subsection{Properties of GASAR Materials}

This section describes the limited literature available on the mechanical and physical properties of GASAR materials ${ }^{1}$. The emphasis is on the mechanical behavior, which is the focus of this thesis, but other properties which have spawned interest in these materials are also presented. There is little data available on the mechanical properties of GASAR materials. No modeling has been done to relate the material properties to the properties of the solid base material or the pore structure.

\subsubsection{Tensile Strengths}

One of the primary interests in GASAR materials has been as a material with a high strength to weight ratio, in terms of both ultimate and yield strengths. The basis of this interest is data presented by DMI that indicates that the addition of pores through the GASAR process leads to a stronger material. This data is shown in Figures 1.5 (yield strength) and 1.6 (ultimate tensile strength) for copper-based GASAR material with longitudinal cylindrical pores tested in the direction of pore orientation. Curves showing the strength of the total cross-sectional area and the net cross-sectional area are shown in each figure. The net cross-sectional area is defined as the solid area in the cross-section and is calculated as

$$
\text { Anet }=\text { Atotal } /(1-p)
$$

where $\mathrm{p}$ is the porosity. The scale on the right side of these graphs shows the strength values in terms of the ratio of porous material strength to solid material strength, while the scale on the left side shows the strength values in absolute terms (MPa). Examination 


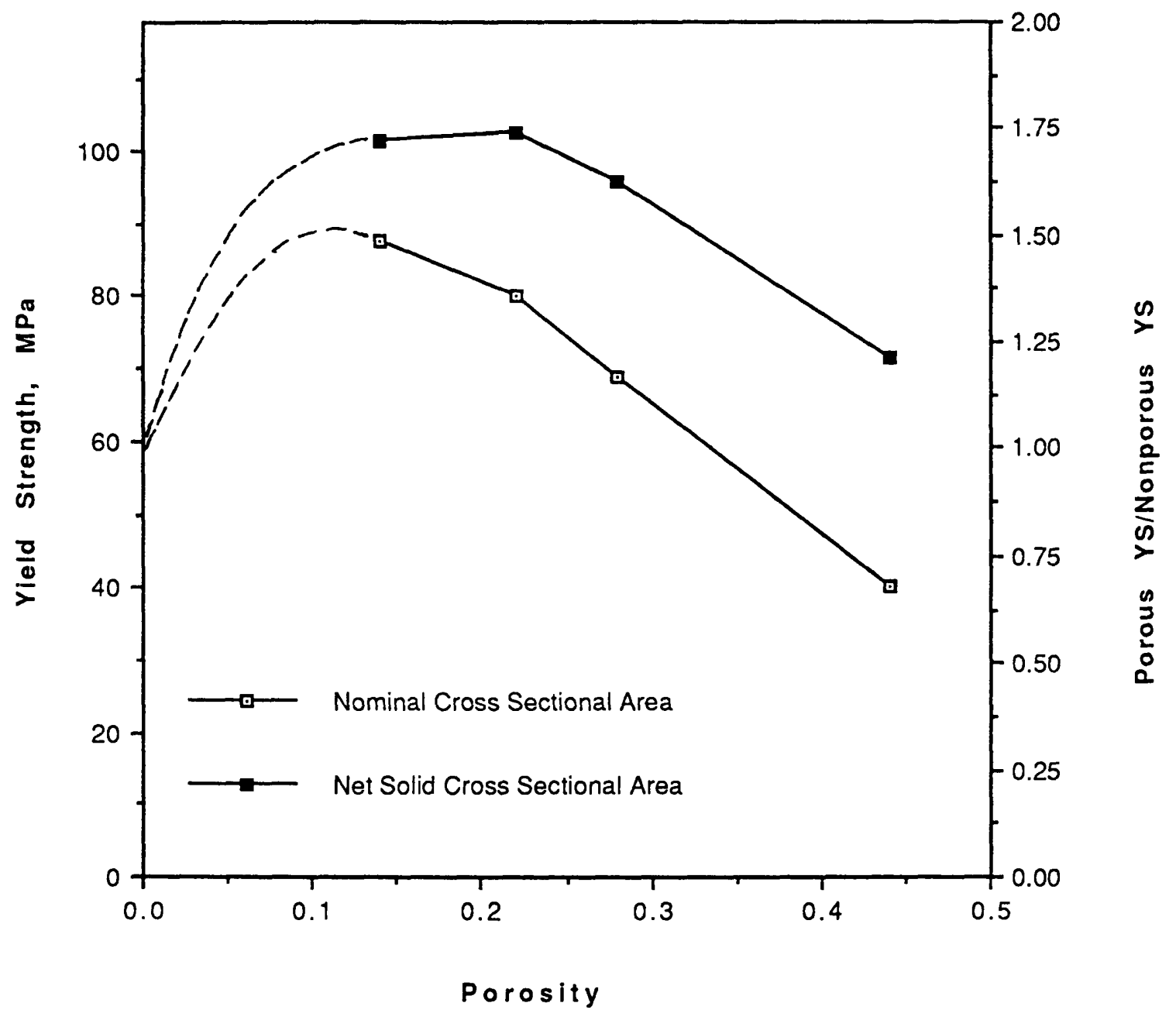

Figure 1.5 - DMI Yield Strength vs. Porosity Data

Data for copper-based GASAR specimens with cylindrical pores oriented in the direction of stress. The method of yield strength determination is unknown. (Ref.1) 


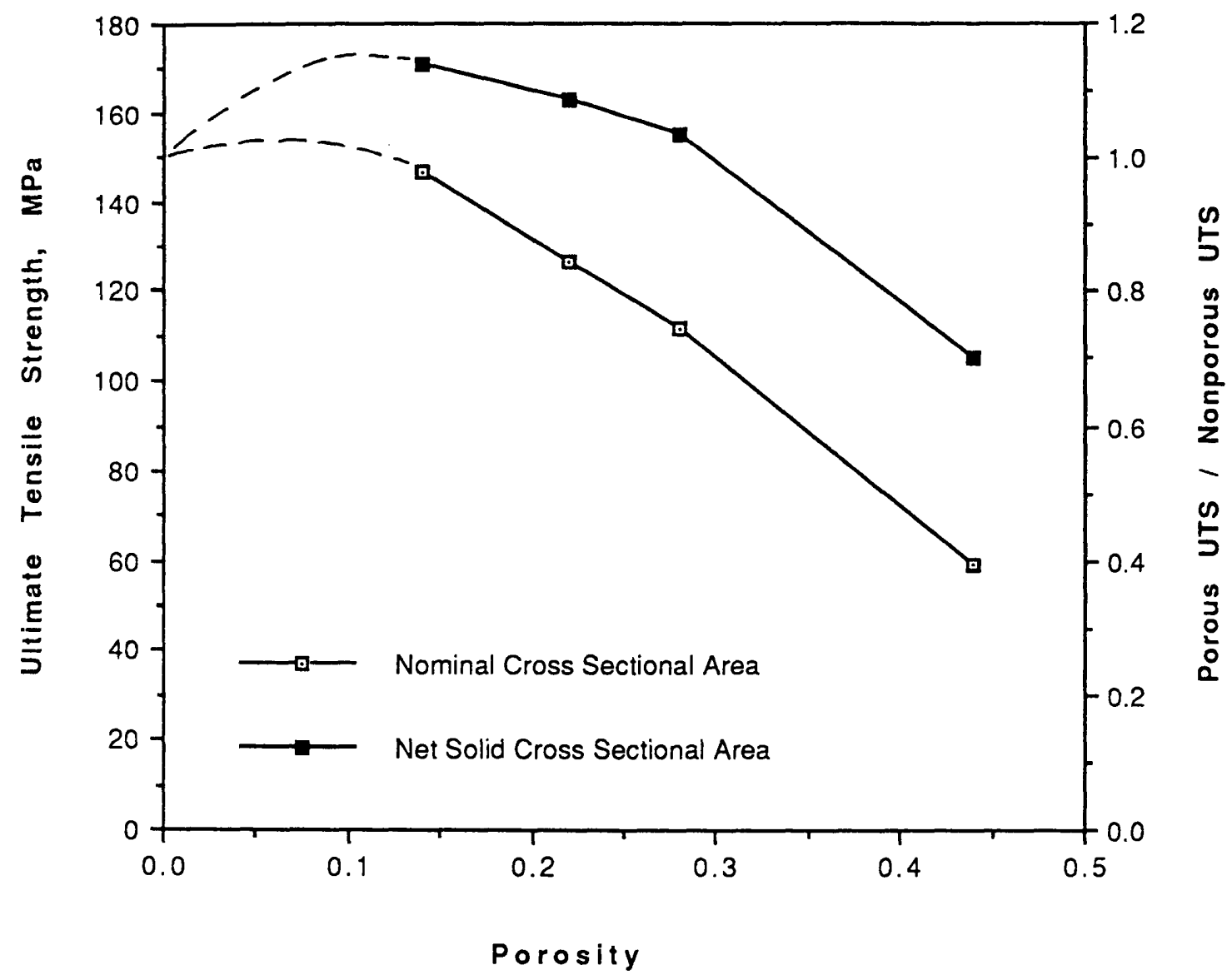

Figure 1.6 - DMI Ultimate Tensile Strength vs. Porosity Data

Data for copper-based GASAR specimens with cylindrical pores oriented in the direction of stress (Ref. 1) 
of these graphs indicate that the yield strength of GASAR porous copper is significantly greater than that of the non-porous copper for porosities up to about 0.33 , and the ultimate tensile strength is greater for porosities up to about 0.12 . This effect is more pronounced when considering the net cross-sectional area. The DMI data indicates that the yield strength of the net cross-sectional area (and thus the yield strength to weight ratio) can be increased by as much as $70 \%$ through GASAR processing. A similar increase of $15 \%$ is indicated in the ultimate tensile strength. The claim that a material can be strengthened by the reduction of its net cross-sectional area is a questionable one which requires a closer examination. A similar strengthening effect has been found by DMI in aluminum-based and magnesium-based materials, although no data is available. Tensile tests of nickel-based GASAR materials, however, show no increase in strength with porosity ${ }^{3}$. This indicates that the strengthening effect produced by the GASAR process does not occur with all base materials, and may in fact, be found only in copper, aluminum and magnesium.

Because DMI provides no description of the testing methods, the data presented in Figures 1.5 and 1.6 are not definite. It is not indicated whether the data points represent four individual specimens or are the mean values obtained for a larger number of specimens. Because no error bars are shown, it must be assumed that the curve presented by DMI is based on only four individual tests. Because the data has been presented for marketing purposes, the possibility also exists that these data points represent the highest strength values achieved in a large number of tests. In this case, the DMI curves would represent an upper bound on the yield and ultimate tensile strengths at each porosity. Also, the method of yield strength measurement is not indicated. In materials like copper, which exhibit significant strain hardening, the measured yield strength is highly dependent on the percent offset strain used. 
Another issue which must be considered is the pore size of the specimens used for the DMI tests. According to Figure 1.3, copper-based GASAR specimens of 0.14 and 0.45 porosity cannot be stably produced with the same pore size. This indicates that there may be a significant variation in the pore size of the materials used for testing. This is important because the relationship between pore size and material strength has not been documented.

Figure 1.3 also indicates that stable copper-based GASAR materials cannot currently be produced with a porosity less than $\approx 0.14$. The relationship between porosity and strength in this range (shown with dashed lines in Figures 1.5 and 1.6) is therefore completely speculative. This fact raises questions about the source of the nonporous copper strength values used in normalizing the DMI data. The yield strength value used is $59 \mathrm{MPa}$, as compared to $69 \mathrm{MPa}$ given by the ASM Handbook for pure annealed copper ${ }^{4}$. The ultimate tensile strength value used is $150 \mathrm{MPa}$, while the Handbook gives a value of $221 \mathrm{MPa}$. These discrepancies may indicate significant differences in the base material of the porous GASAR specimens and the non-porous reference material. Such a difference may completely or partially explain the reported strengthening effect. In addition, recent visits by U.S. Naval researchers to the DMI laboratories in the Ukraine have raised serious concerns with quality control in the production of existing GASAR materials.

Other properties of the stress-strain behavior that are of interest in these materials are the elastic modulus and the ultimate strain to failure. Ultimate strain data is available for nickel-based GASAR porous materials ${ }^{3}$. A decrease in ductility with increasing porosity is reported. No elastic modulus or ductility data has been made available for copper-based GASAR materials. 


\subsubsection{Other Mechanical Properties}

DMI has also indicated that GASAR materials exhibit improved resistance to frictional wear and improved vibrational damping. Mechanical wear data is presented in Table 1.2. Note that the data provided by DMI indicates an increase in the frictional coefficient with increasing load. The testing methods used to obtain this data are unknown. No data is presently available for the vibrational damping behavior. It is also reported, though not yet verified, that GASAR materials retain a significant portion of their strength at high temperatures.

\begin{tabular}{|l|l|l|l|l|}
\hline Material & \multicolumn{4}{l|}{ Unit load,kg / mm } \\
\hline \multicolumn{4}{|c|}{1.5} & \multicolumn{3}{l|}{4.5} & 7.5 \\
\hline $\begin{array}{l}\text { Bronze Al-Fe } \\
\text { 9-4 monolith }\end{array}$ & $\begin{array}{l}0.08 \\
25\end{array}$ & $\begin{array}{l}0.15 \\
95\end{array}$ & $\begin{array}{l}0.21 \\
130\end{array}$ & --- \\
\hline $\begin{array}{l}\text { Bronze sintered } \\
\text { with lubricant }\end{array}$ & $\begin{array}{l}0.03 \\
5\end{array}$ & $\begin{array}{l}0.04 \\
50\end{array}$ & $\begin{array}{l}0.07 \\
70\end{array}$ & destruct \\
\hline $\begin{array}{l}\text { GASAR Cu-8.5 Al } \\
\text { (no lubricant) }\end{array}$ & $\begin{array}{l}0.12 \\
5\end{array}$ & $\begin{array}{l}0.12 \\
55\end{array}$ & $\begin{array}{l}0.17 \\
95\end{array}$ & destruct \\
\hline $\begin{array}{l}\text { GASAR Cu-8.5 Al } \\
\text { FFM-65 lubricant }\end{array}$ & $\begin{array}{l}0.06 \\
10\end{array}$ & $\begin{array}{l}0.04 \\
45\end{array}$ & $\begin{array}{l}0.03 \\
65\end{array}$ & $\begin{array}{l}0.03 \\
180\end{array}$ \\
\hline
\end{tabular}

Top - Frictional Coefficient

Bottom - Frictional Heating, ${ }^{\circ} \mathrm{C}$

Table 1.2 Mechanical Wear Tests on GASAR Materials - (Ref. 1)

\subsubsection{Thermal Properties}

Porous materials are generally much less efficient at conducting heat than a solid material of the same base material. Due to the monolithic nature of the GASAR base matrix, GASAR materials exhibit thermal conductivities much higher than traditional 
porous materials. Copper-based GASAR has shown an increase in the thermal conductivity of up to $140 \%$ over other porous coppers. DMI has claimed that GASAR materials can be produced (through control of pore size, shape and orientation) that actually transfer heat more efficiently than similar non-porous material, with increases in the thermal conductivity in the direction of the cylindrical oriented pores up to $50 \%$. One result of this high thermal conductivity is a higher material cooling rate, particularly when surface pores are present. These pores increase the surface area exposed to the ambient temperature. Heated GASAR samples reportedly emit heat up to five times faster than solid materials with conventionally roughened surfaces. ${ }^{1}$ It is important to note that these thermal properties have not been verified in published reports.

DMI attributes the increase in thermal conductivity to heat convection by hydrogen trapped in the oriented cylindrical pores. It has been determined, however, that convection has a significant role in thermal conductivity only when the ratio of the buoyant convection force to the viscous resistance force, called the Grashof number, is greater than $1000^{5,6}$. It can be shown that for hydrogen-filled pores under normal atmospheric conditions, only GASAR materials at the upper limit of possible pore size approach this limit. Also, gases generally have thermal conductivities which are much lower than that of most solid materials. Therefore, any significant increase in the thermal conductivity of GASAR porous materials is more likely due to the properties of the solid cell wall material rather than any direct effect of the gas-filled pores.

\subsection{Applications of GASAR Materials}

New applications of porous materials are constantly being found. The field of porous metals is one with particularly great potential. The most common applications of porous metals are in filters, catalytic substrates, heat exchangers, and in some structural applications. If the claims made by DMI can be substantiated, GASAR materials are sure to be adapted for use in numerous applications. The following sections present the 
applications in which GASAR materials are currently being used, as well as certain structural applications for which GASAR materials would be ideally suited.

\subsubsection{Current Applications}

GASAR materials are currently being utilized in a variety of commercial applications in the former Soviet Union '. These applications have utilized the mechanical and thermal properties of GASAR materials, as well as the oriented pore structure. Nickel-based GASAR materials are being used as filters in high temperature applications in a chemical factory. These filters, composed of material with penetrating cylindrical pores, offer a long working life and can be cleaned with a simple pressure reversal. Nickel-based GASAR has also been used in medical applications, where it is coated with an organic layering material to produce ultrafine filters for insulin. Bronze and Al-bronze bearings used in the food processing industry have been cut from GASAR cylinders with radially oriented pores. These bearings cool quickly and exhibit a high resistance to frictional wearing. Other applications which have utilized the properties of GASAR materials include ${ }^{1}$ :

- High temperature ceramic catalyst supports for rocket and jet engines

- Magnesium-based light-weight structural panels for the Soviet space program

- Oxygenators for water purification

- Transpiration-cooled elements in the combustion chambers of rocket steering boosters

Many U.S. companies have expressed interest in GASAR materials for various industrial applications, but their use will be limited until they can be produced in the U.S. and until their properties are more closely studied.

\subsubsection{Structural Applications}

The structural potential of a material exhibiting behavior such as that shown in Figures 1.5 and 1.6 is tremendous. A porous material which is significantly stronger 
than the solid metal from which it is made would be highly valuable in numerous fields which utilize lightweight structural materials. Even if this strengthening effect (which is highly questionable) is disproven, the GASAR process offers many potential advantages in various structural applications. The upper bound performance of a cellular material is the out-of-plane behavior of an ideal honeycomb (see section 1.5). GASAR shows the potential to produce a honeycomb-like material with controlled cell size and porosity.

The greatest sources of potential of GASAR materials lies in the ability to produce monolithic laminated structures with a higher resistance to delamination than currently produced laminated materials. An obvious application of this feature is the production of lightweight structural sandwich panels with solid faces and a porous core (see Figure $1.4 \mathrm{~d}$ ). The separation of the solid faces by the less dense core greatly increases the moment of inertia of the panel with a small increase in weight, producing a highly efficient structure with respect to bending and buckling s. If composed of a ductile material, the panel will also have a high energy absorption capacity. Such a structure may be used as exterior structural panels in aircraft or in double-hulled ocean vessels, where the combination of high strength per unit weight and high energy absorption would be valuable in resisting impacts. Another promising application is in cylindrical compression elements. It has recently been shown that the buckling resistance of a hollow cylindrical element is significantly increased by the addition of a foam core ${ }^{7}$. The core acts to decrease the harmonic of the buckling mode of the element. The effective length of the cylinder wall with respect to local buckling is therefore increased. It has been shown that a foam core cylindrical element has a higher buckling resistance than a hollow core element of the same weight. This effect is more pronounced when the core contains radially oriented pores (see Figure $1.4 \mathrm{e}$ ). 


\subsection{The Gibson and Ashby Models for Cellular Solids}

Gibson and Ashby have developed models for cellular materials which accurately describe the mechanical behavior of a wide variety of honeycombed and foamed materials 5 . The Gibson and Ashby models relate the mechanical properties of a cellular material to the mechanical properties of the solid cell wall material, the cell geometry, and the relative density of the cellular material. The relative density is defined as the ratio of the cellular material density, $\rho^{*}$, to that of the solid cell wall material, $\rho$ s. The relative density is equal to the volume fraction of solid in the material and the area fraction of solid in the cross-section. GASAR may exhibit a microstructure different from most other cellular materials, but some of the deformation mechanisms of conventional cellular solids may also influence the mechanical behavior of GASAR materials. GASAR materials with cylindrical pores oriented in the direction of testing, such as those upon which the data in Figures 1.5 and 1.6 are based, may deform in a manner which is similar to either a honeycomb loaded out-of-plane or an open or closed cell foam, depending on the length and connectivity of the oriented pores. The Gibson and Ashby models describing the elastic and plastic behavior of these materials in tension are presented in the following sections.

\subsubsection{Out-of-Plane Behavior of Honeycombs}

An ideal honeycomb is an array of identical cells which fit together to form a prismatic, three-dimensional structure. An ideal honeycomb composed of hexagonal cells is pictured in Figure 1.7. Out-of-plane tensile loading results in the uniaxial stretching of each cell wall. The Young's modulus of the honeycomb is equal to the Young's modulus of the cell wall solid, Es, scaled by the solid cross-sectional area, or:

$$
\frac{E_{3} *}{E_{s}}=\left\{\frac{h / l+2}{2(h / l+\sin \theta) \cos \theta}\right\} \frac{t}{l}=\frac{\rho^{*}}{\rho_{s}}
$$


Plastic yielding occurs in a honeycomb under out-of-plane loading when the net stress in the solid cell walls exceeds the yield strength, $\sigma_{y s}$ of the cell wall material. In compression, plastic buckling of the cell wall will most often occur before the full strength of the solid cross-section can be developed. In tension, however, the relationship between the out-of-plane plastic yield strength of the cellular material $\left(\sigma_{\mathrm{pl}} *\right)_{3}$ and the solid cell wall material yield strength, $\sigma y s$, is identical to that stated for Young's modulus:

$$
\frac{\left(\sigma_{p l}\right)_{3}}{\sigma_{y s}}=\left\{\frac{h / l+2}{2(h / l+\sin \theta) \cos \theta}\right\} \frac{t}{l}=\frac{\rho^{*}}{\rho_{s}}
$$

(a)

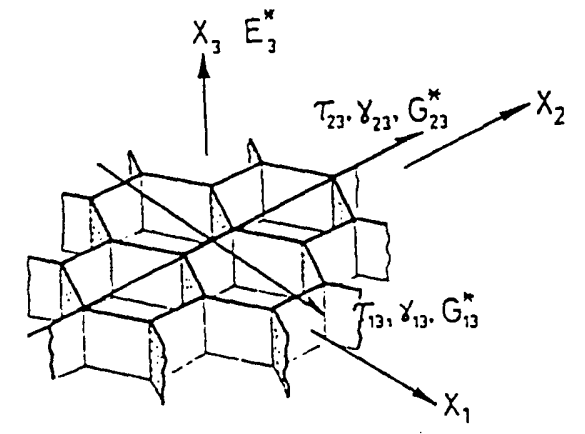

(b)

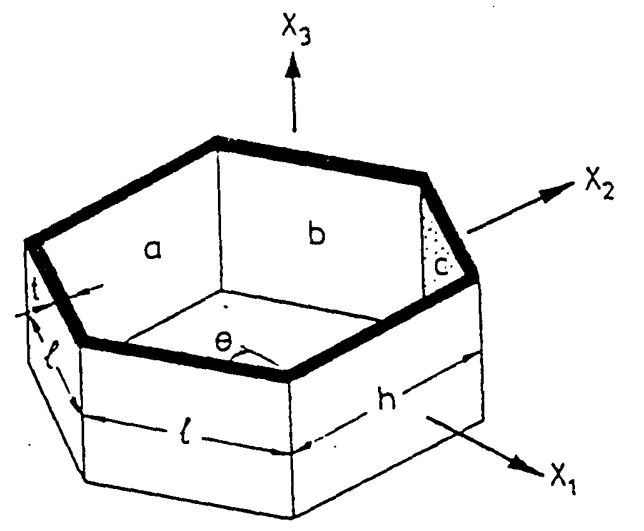

\section{Figure 1.7 - Idealized Honeycomb Cellular Material}

a) The idealized honeycomb structure. $X_{3}$ is the out of plane axis. b) A unit cell of the idealized honeycomb. (Ref. 5) 
In a honeycomb with a ductile cell wall material, the honeycomb will fail when the stress in the solid cell walls exceeds the ultimate tensile strength, $\sigma_{t s}$ of the cell wall material. The out-of-plane failure strength, $\left(\sigma \mathrm{f}^{*}\right)_{3}$ is therefore defined in the same manner as the yield strength and Young's modulus:

$$
\frac{\left(\sigma_{f}^{*}\right)_{3}}{\sigma_{t s}}=\left\{\frac{h / l+2}{2(h / l+\sin \theta) \cos \theta}\right\} \frac{t}{l}=\frac{\rho^{*}}{\rho_{s}}
$$

Thus for honeycombs loaded in out-of-plane tension, the ideal elastic, plastic, and failure properties are all defined by the properties of the solid cell wall material scaled by the relative density of the honeycomb.

\subsubsection{Open-Cell Foamed Materials}

The simplest model for an open-cell foam is a cubic array of solid elements of equal length with a square cross-section, as shown in Figure 1.8. The relative density of the material and the second moment of area, $I$, of an individual element are directly related to the element dimensions by

$$
\frac{\rho^{*}}{\rho_{s}} \propto\left(\frac{t}{l}\right)^{2}
$$

and

$$
I \propto t^{4} .
$$

The initial elastic deformation of an open-cell foam is primarily due to the bending of the cell wall elements. Using elastic beam theory to describe the deformation of these members, the initial elastic modulus of the cellular material is given by

$$
E^{*}=\frac{C_{1} E_{s} I}{l^{4}}
$$




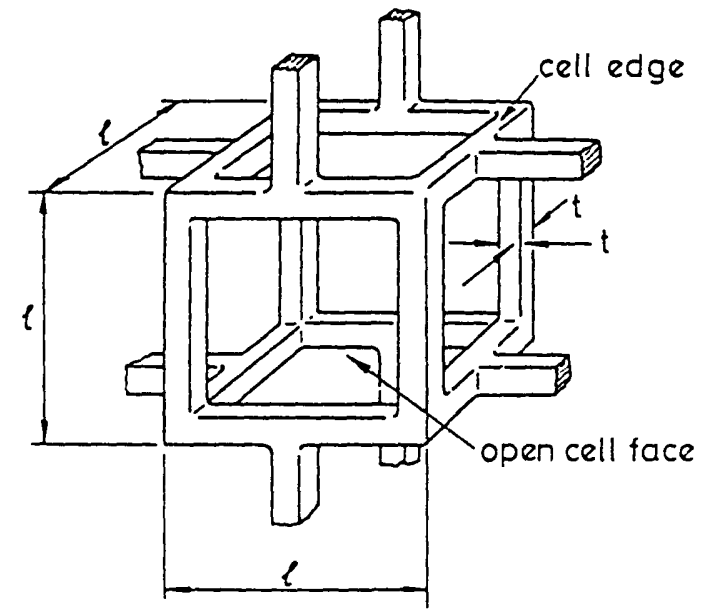

\section{Figure 1.8 - Idealized Open Cell Foam}

A simplified model of the unit cell of an open cell foam. (Ref. 5)

or, using the relationships defined above,

$$
\frac{E^{*}}{E_{s}}=C_{1}\left(\frac{\rho^{*}}{\rho_{s}}\right)^{2}
$$

where $C_{1}$ includes all constants of proportionality. Data has shown that $C_{1} \approx 1$. The slope of the elastic deformation curve in tension will increase at larger strains as elements rotate in bending to be more oriented in the direction of stress.

The plastic yielding strength of an open-cell foam is related to the plastic moment of the individual beam sections, $\mathrm{M}_{\mathrm{p}}$, by:

$$
\sigma_{p l} * \propto \frac{M_{p}}{l^{3}}
$$


The combination of the equation

$$
M_{P}=\frac{1}{4} \sigma_{y s t^{3}}
$$

and the proportionality of the relative density to $(t \Omega)^{2}$ results in the equation

$$
\sigma_{\rho l} *=C_{2}\left(\frac{\rho^{*}}{\rho_{s}}\right)^{3 / 2}
$$

where $\mathrm{C} 2$ contains the proportionality constants. Experimental data has shown that $\mathrm{C}_{2} \approx 0.3$.

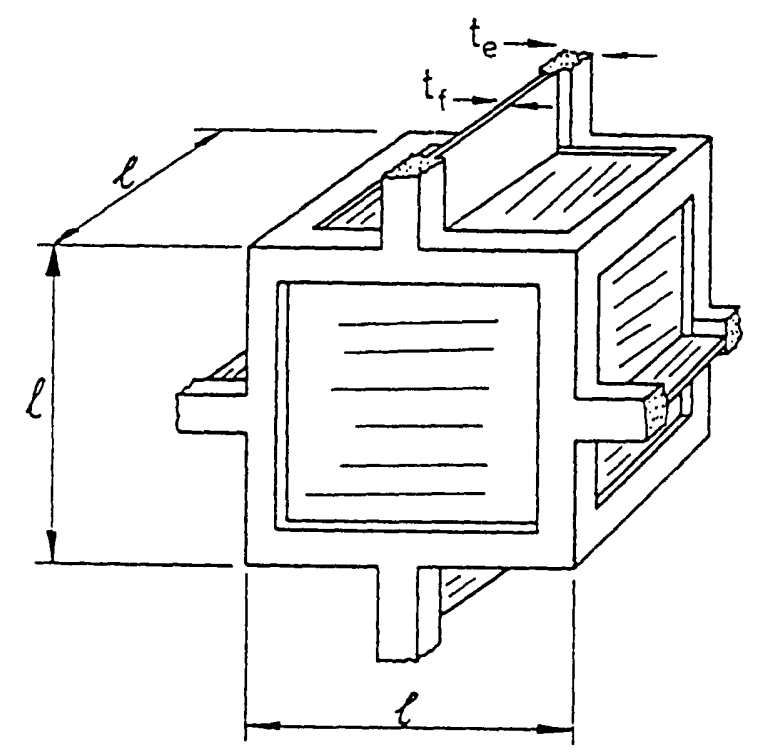

Figure 1.9 - Idealized Closed Cell Foam

A simplified model of the unit cell of a closed cell foam. (Ref. 5) 


\subsubsection{Closed-Cell Foamed Materials}

The model for a closed-cell foam is similar to the open-cell foam of Figure 1.8, except that the cell edges (the square cross-section elements of equal length) contain only a fraction, $\varnothing$, of the total solid material. The remaining fraction, $(1-\varnothing)$, is contained in solid faces which separate the individual cubic cells, as shown in Figure 1.9.

The elastic modulus of closed cell foams is found in the same manner as in opencell foams, although two additional effects must also be considered. The first effect is the stretching of the cell wall material in the direction of stress, and the second is the effect of the gas pressure within the closed cell. These effects are incorporated as extra terms into the ratio of foam modulus to solid modulus. The resulting equation is:

$$
\frac{E^{*}}{E_{s}}=\phi^{2}\left(\frac{\rho^{*}}{\rho_{s}}\right)^{2}+(1-\phi) \frac{\rho^{*}}{\rho_{s}}+\frac{P_{o}\left(1-2 v^{*}\right)}{E_{s}\left(1-\rho^{*} / \rho_{s}\right)},
$$

where Po is the initial gas pressure within the cell, and $\mathrm{v}^{*}$ is the foam's Poisson's ratio $(v \approx 1 / 3)$.

Cell wall stretching and internal gas pressure also influence the plastic yielding stress of a closed-cell foam. The term added for the effect of internal gas pressure is somewhat different due to the fact that yielding involves a larger change in the volume of the cell. The resulting equation is

$$
\frac{\sigma_{p l} *}{\sigma_{y s}}=0.3 \phi^{2}\left(\frac{\rho^{*}}{\rho_{s}}\right)^{3 / 2}+(1-\phi) \frac{\rho^{*}}{\rho_{s}}+\frac{P_{o}-P_{a t m}}{\sigma_{y s}}
$$

where Patm is the atmospheric pressure.

\subsubsection{Anisotropic Unit Cells}

The Gibson and Ashby models also include a provision for anisotropic unit cells such as that shown in Figure 1.10. The ratio of the foam properties in direction 3 to those in directions 1 or 2 is given in terms of the shape-anisotropy ratio, $R=(h / 1)$. The 
anisotropy ratios of the foam properties are given below. Note that in these equations, the ratio goes to 1 if $(h=1)$. The anisotropy ratio for Young's modulus is

$$
\frac{E_{3} *}{E_{1} *}=\frac{2 R^{2}}{\left(1+(1 / R)^{3}\right)},
$$

while that for the plastic yield strength is

$$
\frac{\left(\sigma_{p l} *\right)_{3}}{\left(\sigma_{p l}\right)_{1}}=\frac{2 R}{1+(1 / R)} .
$$

\subsection{Comparison of Gibson and Ashby Models to GASAR Behavior}

The behavior of the copper-based GASAR material shown by the data in Figures 1.5 and 1.6 is clearly not well described by the Gibson and Ashby models. For all

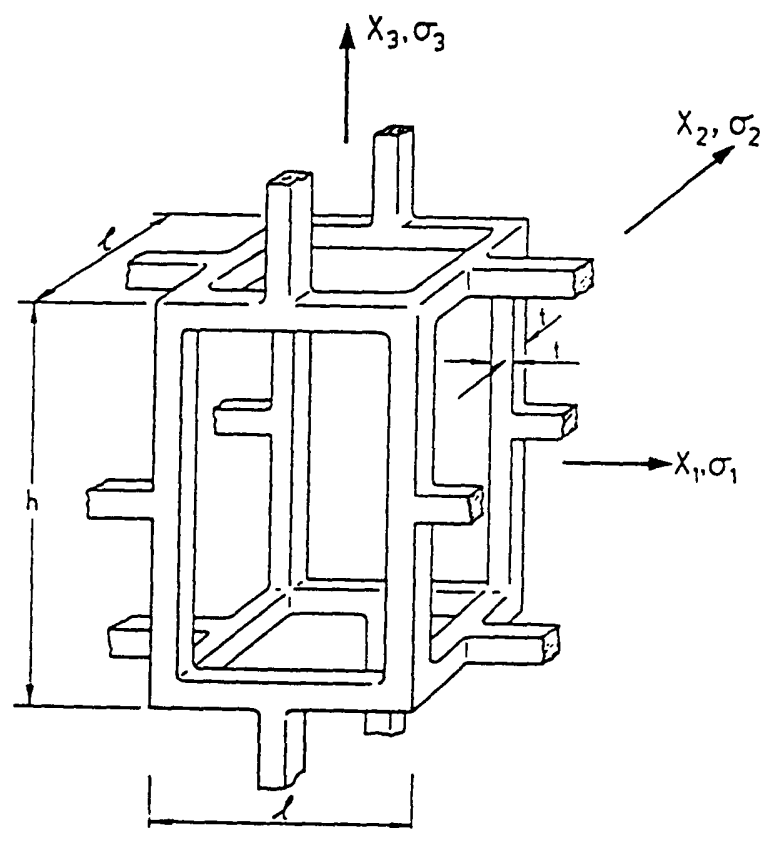

Figure 1.10 - Idealized Anisotropic Foam

The elongated unit cell of an anisotropic foam. (Ref. 5) 
porosity values above zero, the models of honeycombs, open-cell foams and closed-cell foams show a decrease in both yield strength and ultimate tensile strength from the properties of the solid cell wall material. There is no mechanism of deformation considered by the Gibson and Ashby models which would cause the strength of a material to increase with increasing porosity. The existence of a localized triaxial state of stress in the cell walls of the porous material could cause a strength increase. Any impediment to dislocation movement could also have an effect.

Considering that the strength values used by DMI for nonporous copper are significantly lower than all reference values for solid copper, the most likely explanation is that the solid cell wall material in the GASAR porous specimens is somehow different than the nonporous solid material. Such a difference could be due to a variation in the forming process, grain structure, crystallographic texture or impurity content. The properties of copper, particularly the yield and tensile strengths, are highly dependent on the forming processes and heat treatments to which the material has been subjected ${ }^{4}$. Copper exhibits a high degree of ductility and strain hardening. Hot or cold working of the material is the primary forming process. Little data is available for as-cast, high purity copper materials.

\subsection{Related Topics}

This section provides background information on a number of topics which must be addressed in the investigation of copper-based GASAR material properties. The mechanism of deformation of face-centered cubic (FCC) metals is presented. A variety of effects that could influence the yield strength and/or ultimate tensile strength of the solid cell wall metal are also discussed. 


\subsubsection{FCC Crystal Structure and Slip Systems}

The unit cell of the FCC crystal structure is shown in Figure 1.11. The movement of crystal lattice imperfections, or slip, is the basis of plastic deformation in ductile metals. The closeness of atomic packing, as dictated by the crystal structure, directly affects the energy required to propagate slip in any particular crystallographic plane or direction. Thus slip preferentially occurs in the closest packed directions of the closest packed planes. In FCC metals, the crystallographic plane of closest packing is the [111] plane and the closest packed directions are the $<110\rangle$ type directions contained within this plane. There are four [111] planes contained in the FCC structure, and each of these contain three close packed $\langle 110\rangle$ directions ${ }^{8}$. FCC metals thus have a total of 12 independent slip systems. In yielding, slip will first occur in the slip system with the highest relative shear stress.

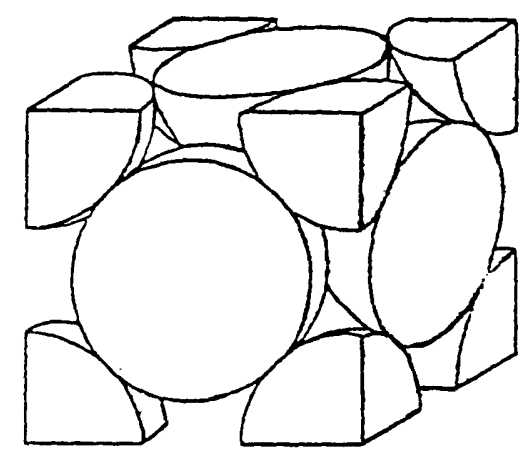

(a)

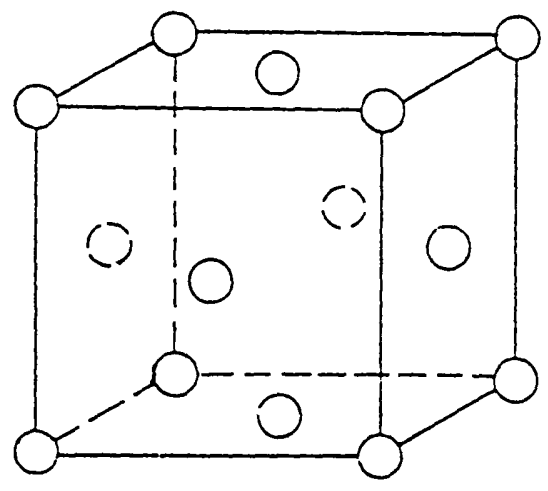

(b)

\section{Figure 1.11 - Unit Cell of FCC Metals}

a) A hard sphere unit cell representation and b) a reduced sphere unit cell representation of the crystal lattice structure of a face-centered cubic metal. (Ref. 8) 


\subsubsection{Yield Criteria}

The yield strength of a material is defined as the stress at which a homogeneous material yields under uniaxial loading conditions. Highly non-uniform and anisotropic materials may be subject to highly localized biaxial or triaxial states of stress due to interaction of the different phases, even under a uniaxial applied stress. Although many theories of yield criteria have been developed to describe such stress states, the most widely accepted with respect to ductile metals is the von Mises yield condition.

The von Mises yield condition is based on distortional energy theory. The total elastic energy is divided into two parts, that associated with volumetric distortion and that associated with shear distortions. The yield criterion is formed by equating the shear distortion energy at yielding under uniaxial stress to that under a combined stress state 9 . The resulting criterion, when stated in terms of the principal stresses, is

$$
\left(\sigma_{1}-\sigma_{2}\right)^{2}+\left(\sigma_{2}-\sigma_{3}\right)^{2}+\left(\sigma_{3}-\sigma_{1}\right)^{2}=2 \sigma_{y p}{ }^{2},
$$

where $\sigma_{y p}$ is the yield point under uniaxial stress. This corresponds to a cylinder in three dimensional $\left(\sigma_{1}, \sigma_{2}, \sigma_{3}\right)$ space with its central axis passing through the origin and having directional cosines of $1 / \sqrt{3}$ (see Figure 1.12 a). For conditions of plane stress, where $\sigma_{3}=0$, the von Mises criterion can be normalized in the form:

$$
\left(\frac{\sigma_{1}}{\sigma_{y p}}\right)^{2}-\left(\frac{\sigma_{1} \sigma_{2}}{\sigma_{y p}^{2}}\right)+\left(\frac{\sigma_{1}}{\sigma_{y p}}\right)^{2}=1,
$$

which is an ellipse corresponding to the intersection of the above cylinder and the $\sigma_{1,} \sigma_{2}$ plane (see Figure 1.12 b). It can be clearly seen that a triaxial or even plane stress state can potentially increase the stress required to initiate yielding in a particular direction. 

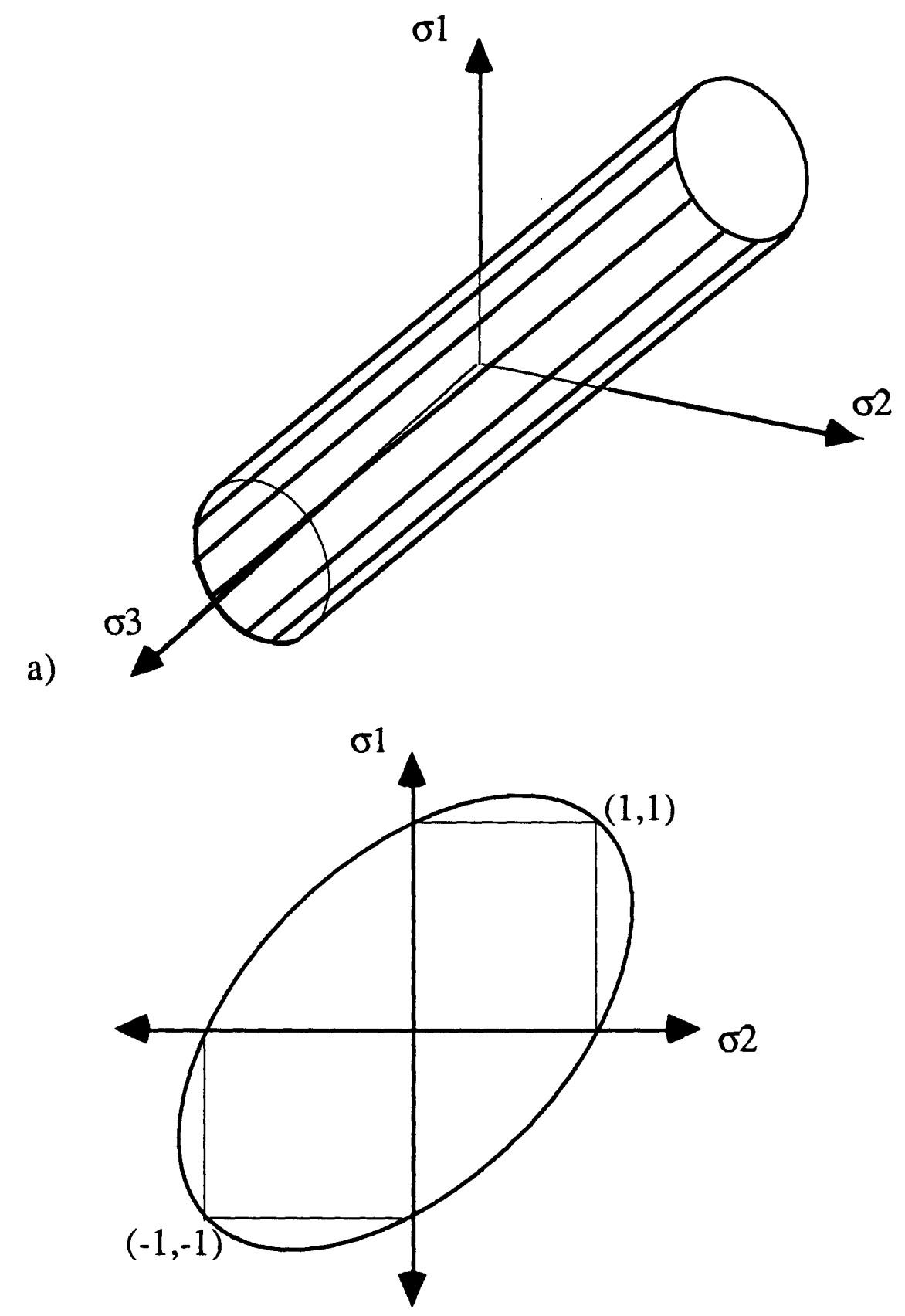

b)

\section{Figure 1.12 - The von Mises Yield Criterion}

a) The von Mises criterion yield surface for a triaxial stress state. b) The yield condition for a plane stress state. (Ref. 9) 


\subsubsection{Unidirectional Solidification}

The concept of subjecting a liquid metal to a directional cooling gradient during solidification is, by no means, a new concept. Directional solidification was used in the 1930 's and 1940's by L. Northcott to investigate its effects on copper alloys ${ }^{10,11}$. In the early 1960 's, unidirectional solidification began to be used with metal alloys in an increasing number of engineering applications, most of which took advantage of improved high temperature mechanical behavior and directional magnetic properties 12,13 . These materials have columnar grains which are oriented in the direction of cooling and which have similar crystallographic orientations (see section 1.7.4). The change in mechanical properties is due to both the grain structure and to a sub-grain dendritic structure composed of two solid phases of different compositions. Directional dendrites form parallel to the temperature gradient and function in a way similar to fibers in fiber reinforced composites (although with less efficiency). Because both phases are solid in these materials, the large body of work related to the strength of unidirectionally solidified alloys cannot be directly applied to GASAR materials. These studies may, however, be applied to research involving the processing and microstructural development of GASAR materials. Northcott ${ }^{11}$ and Eady et al. ${ }^{14}$ study the effect of columnar grains on the yield strength in single phase, low alloy materials, but these studies are not extensive enough to provide a definitive relationship.

The unidirectional solidification of single phase materials may cause a sub-grain microstructure consisting of low angle grain boundaries ${ }^{15,16}$. These low angle, or tilt, boundaries are the result of the dendritic nature of the solidification front and represent the interface of solidified dendrites which stemmed from the same seed crystal. The difference in orientation between these dendrite sub-grains usually ranges from a few minutes to 1 full degree ${ }^{15}$. It has been shown that a well developed substructure of lowangle boundaries can significantly increase the yield strength in steel without a great loss 
of ductility ${ }^{16}$. It has also been shown that the atmospheric pressure at solidification affects the fineness of the resulting substructure ${ }^{17}$. Higher pressures produce a more refined substructure.

\subsubsection{Preferred Orientation}

The existence of crystallographic texture, or preferred orientation, in polycrystalline materials, has been shown to have an effect on mechanical properties. Unfortunately, the vast majority of experimental work done to relate texture to strength involves the texture produced during cold or hot working, called deformation texture 18.19. The results of the experiments involving copper-based materials vary somewhat, presumably due to the dependence of copper strength on processing and heat treatment. Numerous theoretical studies relate material yield strength to the degree and direction of preferred orientation ${ }^{20,21}$. These studies are based on the Taylor theory of plastic deformation in polycrystals, which states that five independent slip systems must be active within a single grain in order for slip to occur ${ }^{22}$. This theory is based on the assumption that continuity must be maintained at the grain boundaries. There has been little experimental work done to directly support this application of Taylor's theory to crystallographic texture which is not the result of deformation. Also, Taylor's theory does not take into account the Hall-Petch effect of grain size, which may be significant in GASAR materials (see section 1.7.6).

It has been shown that the unidirectional solidification of metals with a facecentered cubic crystal structure normally leads to a preferred $<100\rangle$ orientation in the direction of solidification. According to the studies based on Taylor, such a material would be approximately $20 \%$ weaker than a similar material with random crystal orientation. One study of copper single crystal strength vs. orientation showed a higher critical resolved shear strength in crystals tested parallel to the $<100>$ direction, but the method of computation of the shear stress in this study is unclear. Also, single crystals 
loaded at $<100>$ tend to exhibit cross-slip more than other orientations ${ }^{23}$, which could alter the results of such a test. In contrast to the strength values, the relationship of the modulus of elasticity to crystallographic orientation is well documented by numerous sources, both experimentally and theoretically. Unlike the yield strength, the elastic modulus of a metal is not significantly influenced by the grain size or degree of working. In FCC metals, the $<100>$ direction has the lowest modulus, while the $<111>$ has the highest 24,25 . The $<111>$ modulus value can be as much as three times greater than the $<100>$ modulus.

\subsubsection{Impurities Effects}

Recent visits by U.S. researchers to the DMI laboratories in Ukraine have raised questions about the level of quality control in the production of GASAR materials. The addition of impurity elements during processing is a distinct possibility. It is obvious that the magnitude of the effect of a trace alloying element would depend upon the element in question, but in general terms, reported effects of small amounts of impurities on material strength vary somewhat. The ASM Metals Handbook reports similar yield and tensile strength values for annealed wrought coppers of $99.8 \%$ purity and above ${ }^{4}$. No data is given for as cast coppers with a purity above $99 \%$. Rosi ${ }^{26}$, however, reports a nearly $50 \%$ increase in the critical resolved shear strength from $99.999 \%$ to $99.98 \%$ pure copper single crystals. The impurity material involved in this study is unknown.

The possible effect of solid solution hydrogen remaining from the GASAR solidification process must be considered. The effect of hydrogen inclusion in solid copper has been shown to be a temperature-dependent effect ${ }^{27}$. A significant increase in the yield strength of copper has been found at temperatures between $77 \mathrm{~K}$ and $200 \mathrm{~K}$. No such increase was seen at room temperature. 


\subsubsection{The Hall-Petch Effect}

The grain size of a polycrystalline material can have a significant effect on its yield strength. In a polycrystalline material subject to a uniaxial load, slip will occur first in those grains containing slip planes close to $45^{\circ}$ from the loading direction ${ }^{16}$. As these grains slip, the grain boundaries act as impediments to dislocation movement. Since the stress field around dislocations also impedes the approach of other dislocations, those traveling on the same slip plane begin to "pile-up" at the boundary. A shear stress concentration forms in the adjacent grain at the head of this pile-up. The magnitude of this stress concentration is proportional to the square of the pile-up length (from crack theory). This stress concentration eventually "unlocks" less favorably oriented dislocations in the adjacent grain. The length of the pile-up is assumed to be the grain diameter. The yield stress of the polycrystalline material can therefore be defined as

$$
\sigma_{\mathrm{ys}}=\sigma_{\mathrm{o}}+\mathrm{kD}^{-1 / 2},
$$

where $\sigma_{\mathrm{ys}}$ is the yield stress, $\mathrm{D}$ is the mean grain diameter, and $\sigma_{\mathrm{o}}$ and $\mathrm{k}$ are material constants. This relationship has been verified for copper ${ }^{28,29}$, although the values obtained for the material constants vary somewhat (probably due to differences in the impurity level or forming process). The effects of preferred orientation and grain shape on the Hall-Petch effect are unknown.

In GASAR materials the variation of pore size and porosity may have a significant effect on the grain size of the solid cell wall material. The relationship between the pore structure and the grain structure is unclear. The grains could be very small with respect to the pores, or could be much larger, with one grain containing a number of pores (see Figure 1.13). 


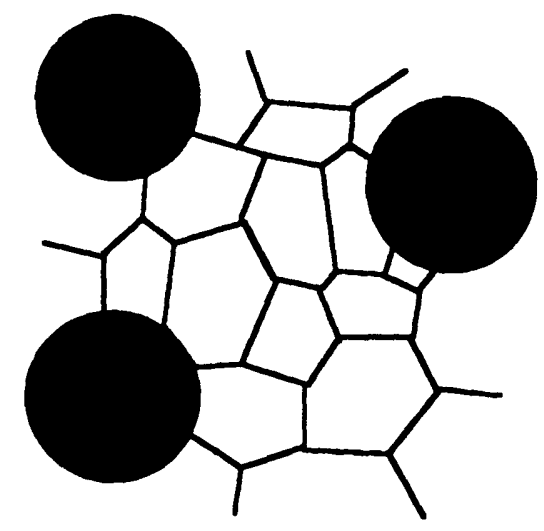

a)

b)

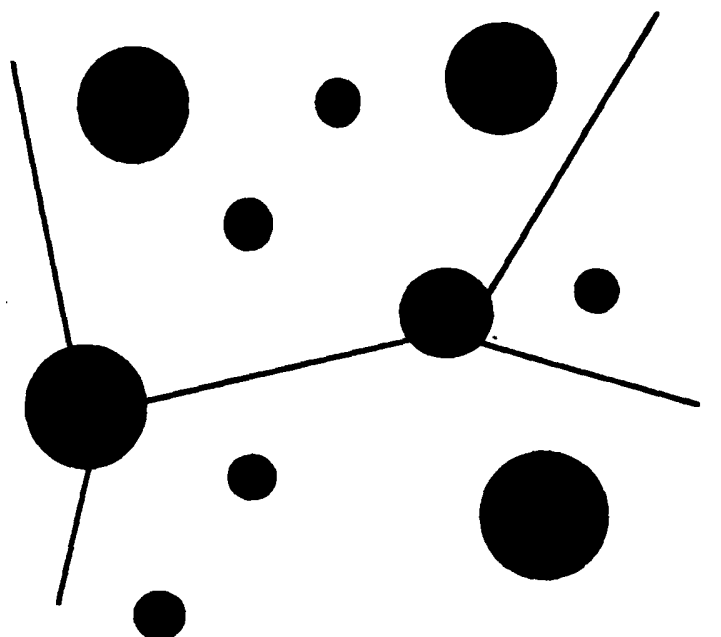

\section{Figure 1.13 - Relationship Between Pore and Grain Size}

The relative size of the pores and grains in a material affect the resulting microstructure. In a), the pores are larger than the grains, and several grains are found between adjacent pores. In $\mathrm{b}$ ), the grains are much larger and a single grain may contain many pores.

\subsubsection{Specimen Size Effects}

Although the grain size of GASAR materials has not been determined, cast metals that have been cooled directionally generally contain large grains ${ }^{10,11,14,17}$. Because the size of the specimens used by DMI is unknown, it is possible that the grain size is close to the specimen diameter. Taylor's theory of plasticity in polycrystals is based on the requirement for continuity at the grain boundaries ${ }^{22}$. This requirement necessitates the activation of five independent slip systems to be active in order for slip to occur, which raises the complexity of the deformation mode and thus increases the strength. This fact explains the large difference between the single crystal strength of a material and its polycrystalline strength. As the grain size increases in relation to the specimen size, a larger percentage of the grain boundary is composed of free surface than solid interface, 
which decreases the amount of constraint on each grain. This effect has been shown by Hansen ${ }^{30}$ to be significant in polycrystalline aluminum with a specimen to grain diameter ratio of less than 15 .

\subsection{Thesis Goals}

Little information is available on the mechanical properties of porous materials made using the GASAR process. The goal of this research project is to characterize the microstructure and uniaxial behavior of porous GASAR materials. We aim to model the material as a cellular solid using the models of Gibson and Ashby (section 1.5). In this thesis, the focus will be on porous copper with cylindrical voids of high aspect ratio.

Existing models for the mechanical behavior of cellular materials indicate that the mechanical properties should decrease as the porosity increases. The GASAR material with cylindrical voids with high aspect ratio can most appropriately be modeled as a honeycomb-like structure; we then expect the mechanical properties to decrease linearly with increasing porosity.

Our specific aims are:

to measure the uniaxial tensile and compressive response of porous copper GASAR material with cylindrical voids aligned with the loading direction

to characterize the microstructure of the material (i.e. porosity, pore size, grain size, orientation of grains, composition)

-to model the material as a cellular solid to describe its mechanical behavior -to explain the apparent increase in strength with increasing porosity

\subsection{Thesis Structure}

Chapter two presents and discusses the experimental techniques and procedures. Chapter three presents the results of all testing. Chapter four presents a discussion of the results. Chapter five summarizes the conclusions reached and makes recommendations for continuing research. 


\section{Chapter Two: Experimental Methods}

The following chapter descibes the materials used for testing and the testing methods and procedures. The tests described in this section include uniaxial tensile tests, microscopic examination of microstructural features, texture analysis, and impurity content analysis.

\subsection{Materials}

One of the goals of the this thesis is to verify the DMI yield strength and ultimate tensile strength data shown in Figures 1.5 and 1.6. It was therefore necessary to obtain copper-based GASAR specimens with longitudinally oriented cylindrical pores, similar to those used in the DMI tests. All GASAR specimens were obtained from the Ukrainian laboratories through a U.S. based subsidiary (DMK Technologies, Ann Arbor, MI). Specimens with pore sizes of $10 \mu \mathrm{m}, 50 \mu \mathrm{m}$, and $500 \mu \mathrm{m}$ were ordered with porosities of $0.1,0.2,0.3,0.4$ and 0.5 . Due to the limited oven configurations currently available at the Ukrainian labs, porous specimens were available only in cylindrical ingots $75 \mathrm{~mm}$ long and $10 \mathrm{~mm}$ in diameter. Specimens of the nonporous material used in the DMI tensile tests were also ordered. The nonporous copper was only available in cylindrical ingots $100 \mathrm{~mm}$ long and $50 \mathrm{~mm}$ in diameter, which is significantly larger than the size of the porous specimens. The nonporous copper samples were produced by unidirectional solidification in an argon-base atmosphere [DMI, personal communication] in order to prevent pore nucleation. 


\subsection{Uniaxial Tensile Tests}

Uniaxial tension tests were performed by loading machined specimens at a constant rate of deformation on an Instron testing frame. The deformation of the specimen over a defined gage length and the total load carried by the specimen were recorded as a function of time. This data was used to construct stress-strain curves using the engineering stress, $\sigma$, and the engineering strain, $\varepsilon$ :

$$
\sigma=\frac{P}{A_{o}} \quad \text { and } \quad \varepsilon=\frac{\Delta L}{L_{o}} \text {, }
$$

where $\mathrm{P}=$ load, $\mathrm{Ao}=$ original cross-sectional area of the specimen, $\mathrm{Lo}=$ original gage length, and $\Delta \mathrm{L}=$ change in gage length ${ }^{16}$.

The properties to be measured from the stress-strain curve are: Young's modulus, yield strength, ultimate tensile strength and ductility. The elastic modulus, or Young's modulus, was measured according to ASTM specifications as the slope of the stress-strain curve below the proportional limit ${ }^{31}$. Because copper does not show a well defined yield point due to strain hardening, the yield strength was measured using the offset method ${ }^{16,32}$, using an offset strain of 0.002 , or $0.2 \%$. The ultimate tensile strength was measured as the peak stress point on the stress-strain curve. The strain at which the peak stress (ultimate tensile stress) occured was recorded as a measure of the specimen's ductility. This value was recorded instead of the ultimate strain because many specimens warped during strain softening after the peak stress was reached and showed a tearing failure that prevented an accurate measurement of the ultimate strain (see section 3.2.5). The strain measured during this warping and tearing behavior would be highly dependent on the position of the measurement device.

\subsubsection{Specimen Design}

Waisted cylindrical specimens were used in the tensile tests. ASTM standards require a ratio of reduced diameter to gage length of 5 for specimens machined from metal 
rods ${ }^{32}$. For various reasons, it was decided to perform the microstructural characterization on specimens from the unreduced length of the tensile specimens. Therefore, the unreduced length on each end had to allow for both an effective gripping area and the microscopy specimen. The length between the unreduced end sections had to include the gage length, two transition fillets, and an additional length equal to the reduced diameter to allow for spacing between the fillets and the limits of the gage length. It was decided to use threaded connections to reduce the required development length of the grips as much as possible. The dimensions shown in Figure 2.1 were used in the final design. They are proportional to those of the standard ASTM $12.5 \mathrm{~mm}$ diameter specimen. The $5 \mathrm{~mm}$ gage length diameter is 10 times the largest pore size $(500 \mu \mathrm{m})$. For compatibility of results, tensile specimens of this design were made for both the porous samples and the nonporous material. In order to investigate the influence that specimen size may have on the tensile test results, specimens with a reduced diameter of $12.5 \mathrm{~mm}$ were also machined from the nonporous ingots. Because the specimen dimensions were not severely limited by the size of the nonporous ingot, shouldered specimens were used to avoid possible complications in threading. The design of these specimens is shown in Figure 2.2.

Specimens were machined using a standard metal lathe and tool steel threading dies. A significant amount of tear-out occurred during the threading of the $5 \mathrm{~mm}$ diameter specimens due to the high ductility of the copper base material and the discontinuities created by the pores, but all connections allowed full development of the ultimate tensile strength of the specimen.

\subsubsection{Testing Equipment and Procedure}

All $5 \mathrm{~mm}$ diameter tensile specimens were tested on an Instron (model 4201) test frame at a constant crosshead speed using a $5 \mathrm{kN}$ load cell. A Hyundai data acquisition system monitored and recorded data from the control unit at 1 second intervals. The data 


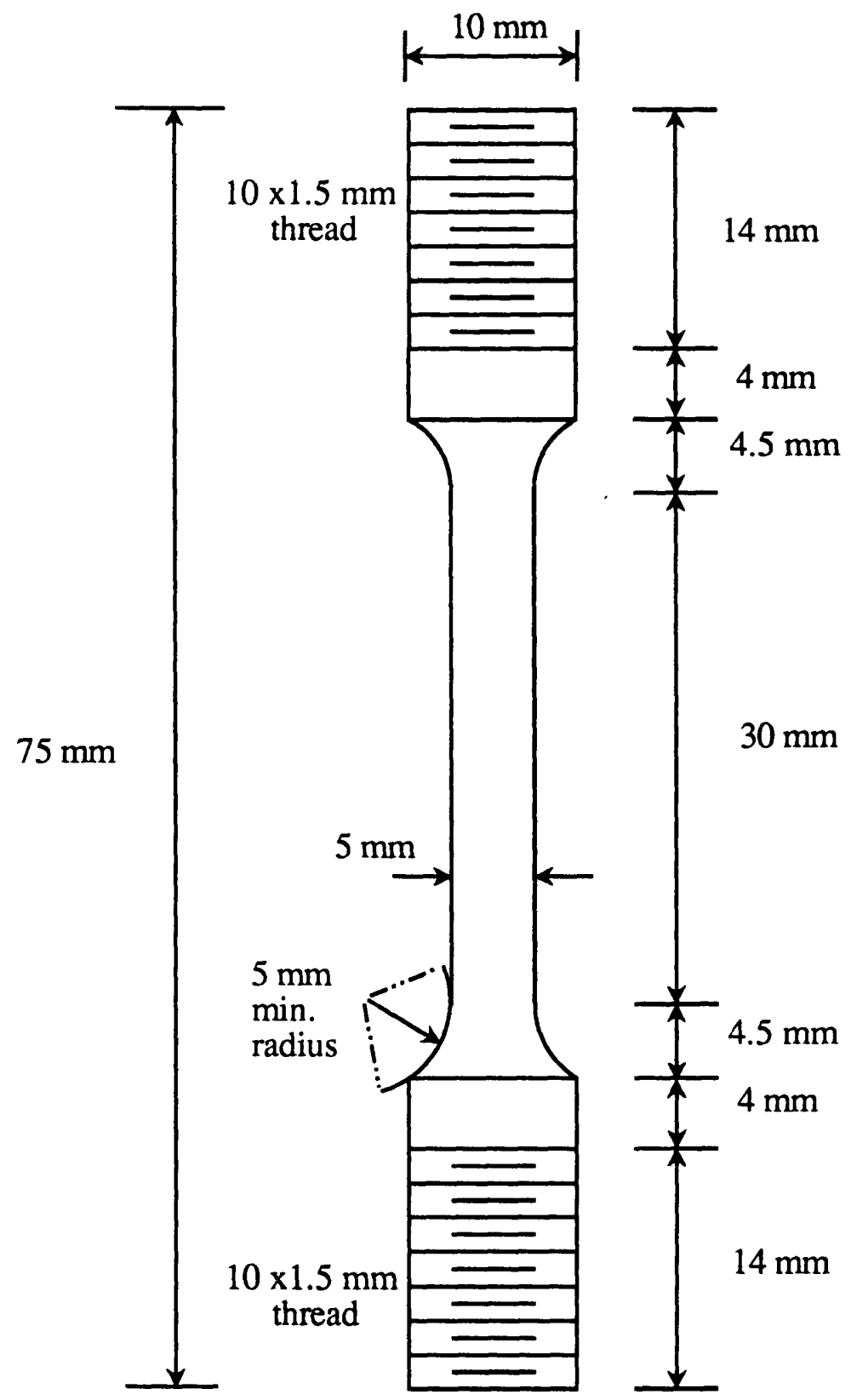

$$
\text { Gage Length }=1^{\prime \prime}=25.4 \mathrm{~mm}
$$

Figure 2.1 - Design of Tensile Specimen (5 mm Diameter) Dimensions were determined according to ASTM E8M, using dimensions proportional to the standard $12.5 \mathrm{~mm}$ specimen. 


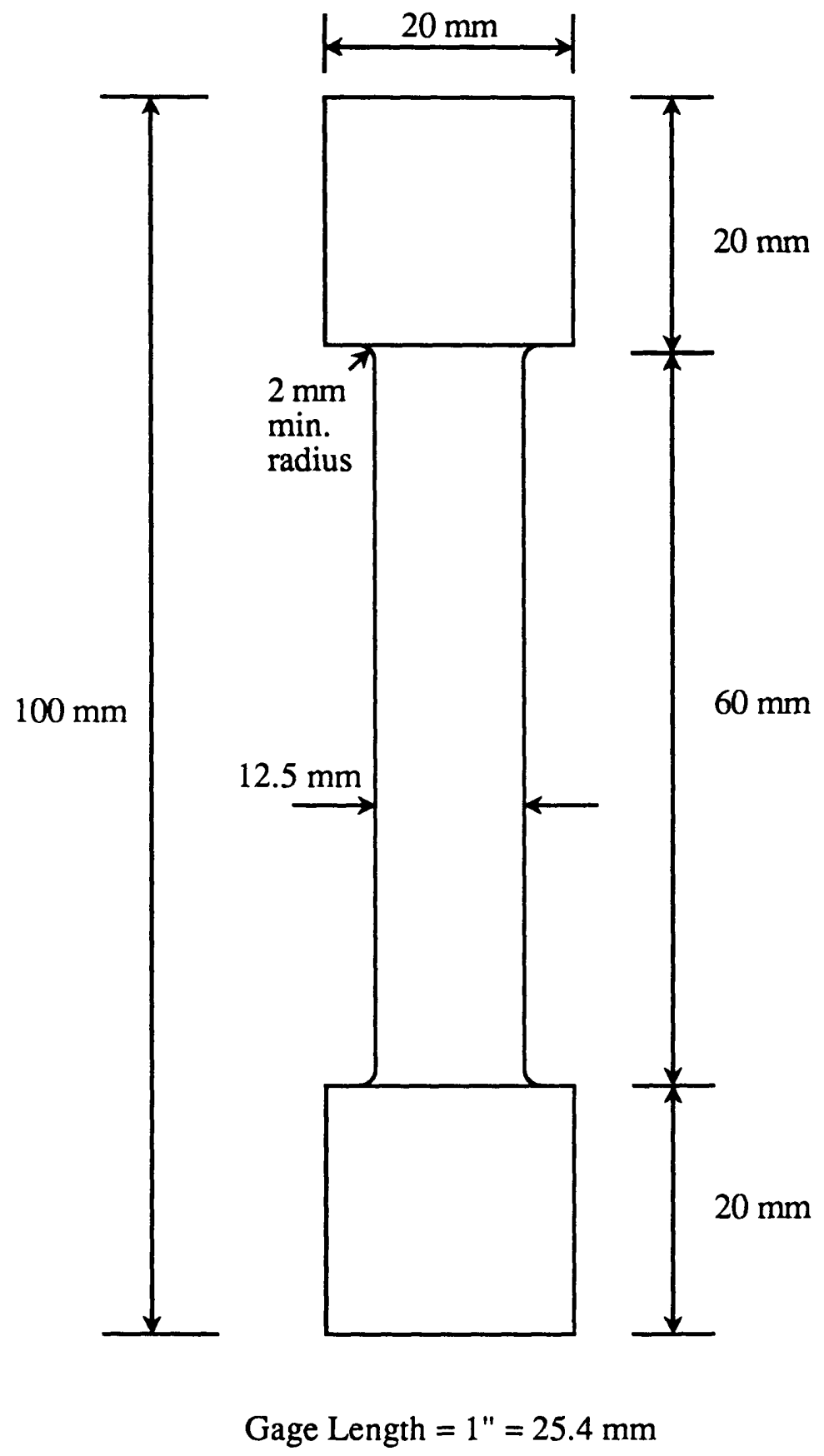

Figure 2.2 - Design of Tensile Specimen (12.5 mm Diameter) Dimensions determined according to ASTM E8M 
recorded included the load (from the load cell), the movement of the crosshead from a preset reference point, and the strain over the gage length, as recorded from a calibrated extensometer. The specimens were connected to the test frame base and crosshead through two universal joints with female threads. The purpose of these joints was to prevent the development of bending stresses from any misalignment that may occur, since such stresses would alter the final test results. A schematic diagram of the tensile testing apparatus with the specimen in place is shown in Figure 2.3.

The $12.5 \mathrm{~mm}$ diameter specimens were tested using an apparatus similar to that used for the $5 \mathrm{~mm}$ diameter specimens. A larger Instron testing frame (model 8500) and a higher capacity load cell $(100 \mathrm{kN})$ were used. The only major difference in the testing methods was the gripping method used. Split cylinder couplings (Figure 2.4) were used to test the $12.5 \mathrm{~mm}$ diameter specimens. These grips also acted as universal joints to prevent the build-up of bending stresses.

Two cantilever arm extensometers, each with a 1" $(25.4 \mathrm{~mm})$ gage length, were used during each tensile test. Because the strains involved in Young's modulus measurements are more than a order of magnitude smaller than the failure strains in copper, both a $10 \%$ elongation and a $100 \%$ elongation extensometer were required to make the accurate measurements of both the elastic and failure strains. The $10 \%$ elongation extensometer was used in straining the specimen to a point well beyond the yield stress. The specimen was then unloaded (the unloading curve also being recorded), and the extensometers changed. The specimen was then reloaded and taken to failure. The plastic strain after unloading was added to the strain measurements made with the second extensometer in order to produce a continuous stress strain curve. In some cases, copper has been observed to form a small yield point upon unloading and reloading ${ }^{33}$, but the increase in stress is negligible in comparison with the stresses involved and has 


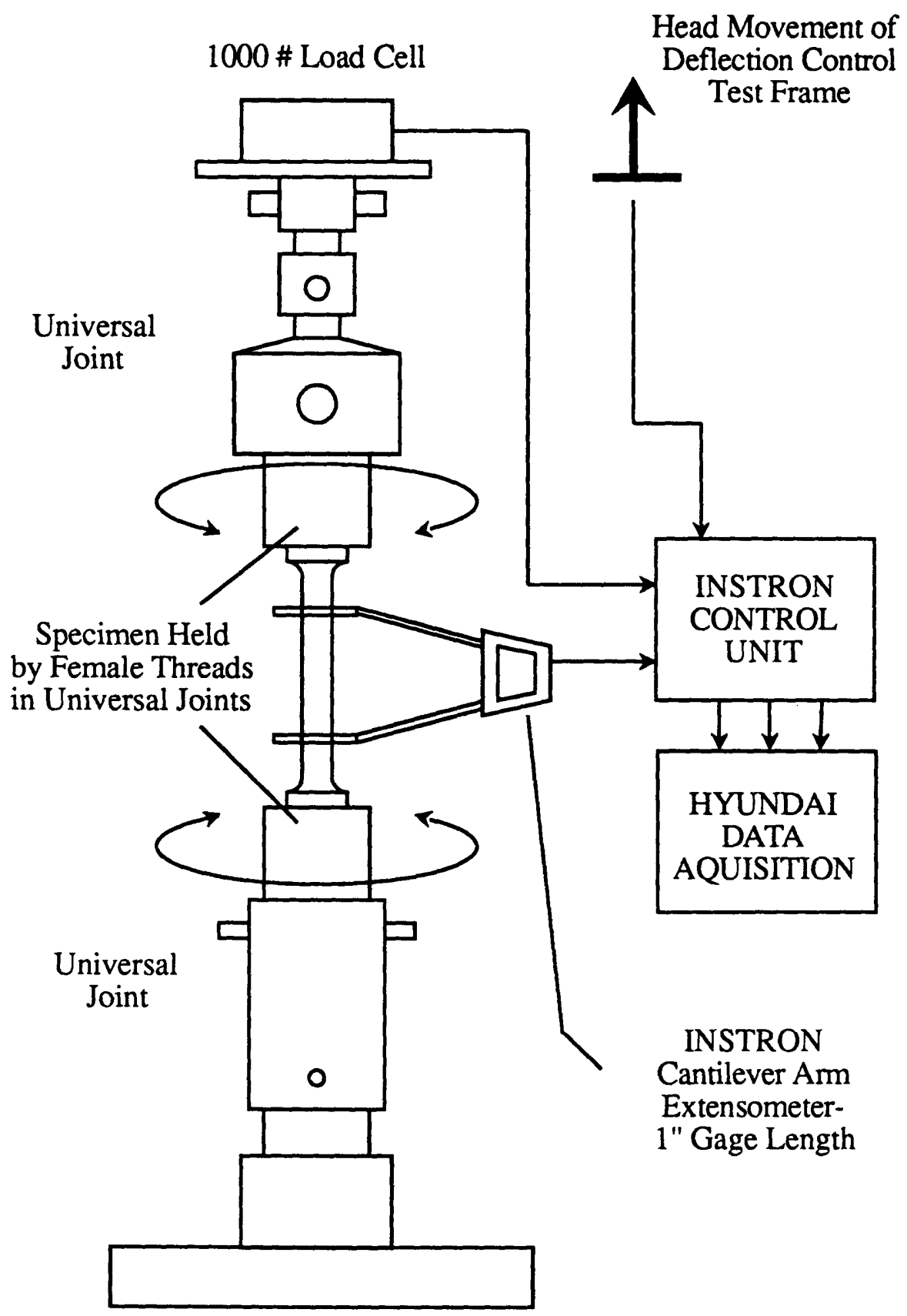

Figure 2.3 - Schematic Diagram of the Tensile Testing Apparatus

The apparatus used for testing of the $5 \mathrm{~mm}$ diameter specimens is shown

A similar set-up was used for testing the $12.5 \mathrm{~mm}$ specimens, but with a larger load cell and the grips shown in Figure 2.5. 
Load Cell / Crosshead

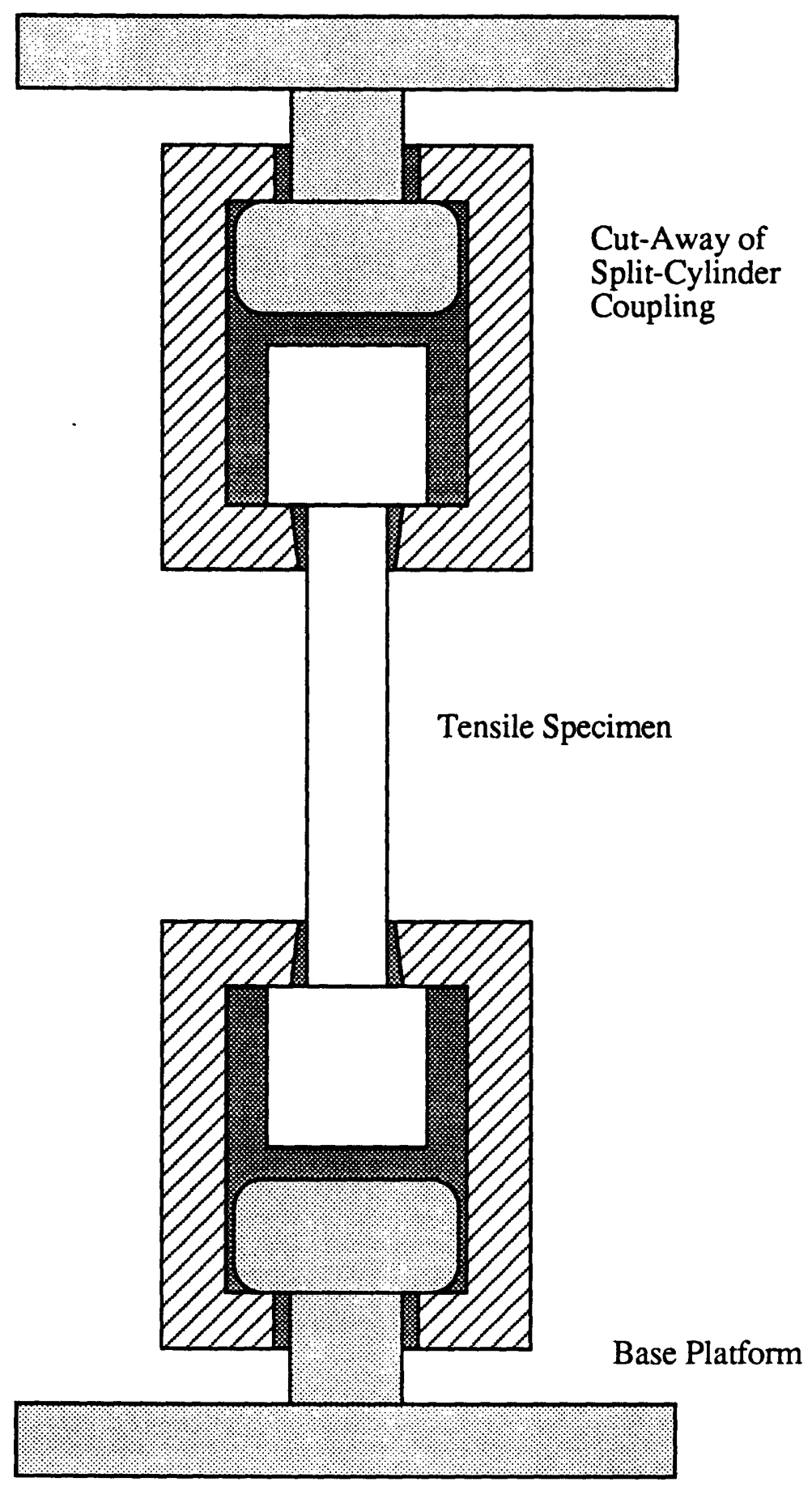

Figure 2.4 - Schematic Diagram of Grips For $12.5 \mathrm{~mm}$ Diameter Specimens

Grips consist of two couplings which connect each shouldered end of the specimen to a shouldered projection from the testing frame. 
no effect on the ultimate tensile strength. No significant discontinuities were found upon assembling the continuous stress-strain curves.

The strain rate of all tests was controlled by the speed of the crosshead. For the 5 $\mathrm{mm}$ diameter specimens, this speed was $0.25 \mathrm{~mm} /$ minute from the beginning of the test to a point well past the yield point. This corresponds to a strain rate of less than 0.0002 $/ \mathrm{sec}$. for a reduced section length of $30 \mathrm{~mm}$. After yielding had occurred, the crosshead speed was increased to $0.5 \mathrm{~mm} /$ minute, or a strain rate of under $0.0004 / \mathrm{sec}$. Such a strain rate increase is permissible under ASTM standards ${ }^{32}$, as long as the increase occurs well after the yield point. The strain rate of the tests on the $12.5 \mathrm{~mm}$ diameter specimens was similarly limited to under $0.0002 / \mathrm{sec}$. until yielding occurred and then under $0.0004 / \mathrm{sec}$. until failure.

\subsection{Microscopy Methodology}

Microscopic examination of the microstructure of the GASAR specimens was done in order to measure the grain size and pore size in both the transverse and longitudinal directions. Observations on the microstructure as a function of porosity were also made. Qualities of sub-grain structure and of the fracture surface of some samples were also noted.

With the exception of the nonporous specimen samples, all microscopy specimens were cut from the $5 \mathrm{~mm}$ diameter tensile specimens after failure, from the approximately $4 \mathrm{~mm}$ long sections on either side of the reduced length between the fillets and the threads. Because of the anisotropy of the porous structure, both transverse (cut perpendicular to the testing direction), and longitudinal (cut parallel to the testing

direction) specimens were made. Cuts were made with a Buehler ${ }^{\circledR}$ low speed diamond wheel saw to minimize damage to the specimen and reduce the amount of polishing necessary. 


\subsubsection{Polishing and Etching Procedures}

The microscopy specimens were highly polished and etched before examination, in order to clarify microstructural features as much as possible. The polishing procedure was based on standard procedures recommended for copper and copper alloys ${ }^{34}$. All work was done on an 8 " polishing/grinding wheel. The procedure consisted of three stages: fine grinding, coarse polishing, and final polishing. Fine grinding consisted of removing surface material damaged from cutting through grinding successively with 240 , 400, and 600 grit silicon-carbide sandpaper discs, using water as a lubricant. Coarse polishing was done with a nylon cloth impregnated with a $5 \mu \mathrm{m}$ aluminum oxide powder. The final polishing was done with a low-nap polishing cloth impregnated with a $0.5 \mu \mathrm{m}$ alumina powder. Water was used as the suspension medium for the polishing powders. For specimens used in any chemical analysis, a diamond paste was substituted for the aluminum oxide and alumina powders so that any residue left in the pores would not be mistaken for true impurities in the solid base material. This was particularly important because it was suspected that alumina may be one of the major impurities, based on analyses performed by other researchers ${ }^{35}$.

Etching is the treatment of a metal surface with an acidic solution in order to examine its microstructure. Grain boundaries and dislocation concentrations are corroded by the acid at a faster rate than the rest of the metal surface, making them clearly visible during microscopic examination. ASTM procedures and safety precautions were followed in the preparation and application of all etchants ${ }^{36,37}$. Two etchants were used in microscopy specimen preparation: a concentrated macroetchant solution and a more dilute microetchant solution. The macroetchant was used for the removal of damaged copper from a specimen's surface. After several trials with different macroetchant solutions, it was found that a $50 \% \mathrm{HNO}_{3}-50 \%$ water solution worked most effectively ${ }^{37}$. The microetchant which was found to produce the best results was a 
solution consisting of $5 \mathrm{~g} \mathrm{FeCl}_{3}, 50 \mathrm{ml} \mathrm{HCl}$, and $100 \mathrm{ml}$ water (ASTM etchant \# 34) ${ }^{36}$. This solution produced well defined grain boundaries and pores.

Due to the high ductility of the copper base material of the porous samples, the pores were often covered over with a thin layer of copper during the coarse polishing stage. After experimentation with different methods, the best results were achieved by 1 ) completing the full polishing procedure, 2 ) removing the deformed surface layer with the macroetchant, 3) repeating the final polishing stage, and then 4) re-etching with the microetchant to bring out the microstructure.

\subsubsection{Microscopic Examination}

Microscopy was performed using both optical and scanning electron (SEM) microscopes. The optical microscope provided clearer views at lower magnifications and was used primarily for views of the pore configuration and arrangements of a large number of pores. The majority of the microscopic examination was done on a Cambridge Instruments SEM. An SEM can reveal topographical details of less than 50 Angstroms with a depth of focus 500 times that of an optical microscope ${ }^{38}$. The heart of the machine is a gun which produces a beam of electrons with energies of from 1 to 50 $\mathrm{keV}$. The beam is condensed into an electron probe by a series of condensing lenses and is directed by deflection coils which scan the beam over the test specimen in a rectangular pattern. The chamber containing the specimen and beam is kept under vacuum. The electron probe produces a variety of signals upon penetrating the surface of the specimen. The signals usually measured in producing an image are secondary electrons, low energy electrons which escape from the specimen at only the uppermost layers; and backscattered electrons, elastically rebounded electrons with a higher energy level. Both types of signals were used at various stages during the work contained in this report. The intensity of these signals varies with the topography of the surface being examined, with a recess producing a much weaker signal than a peak. Detectors are placed in the 


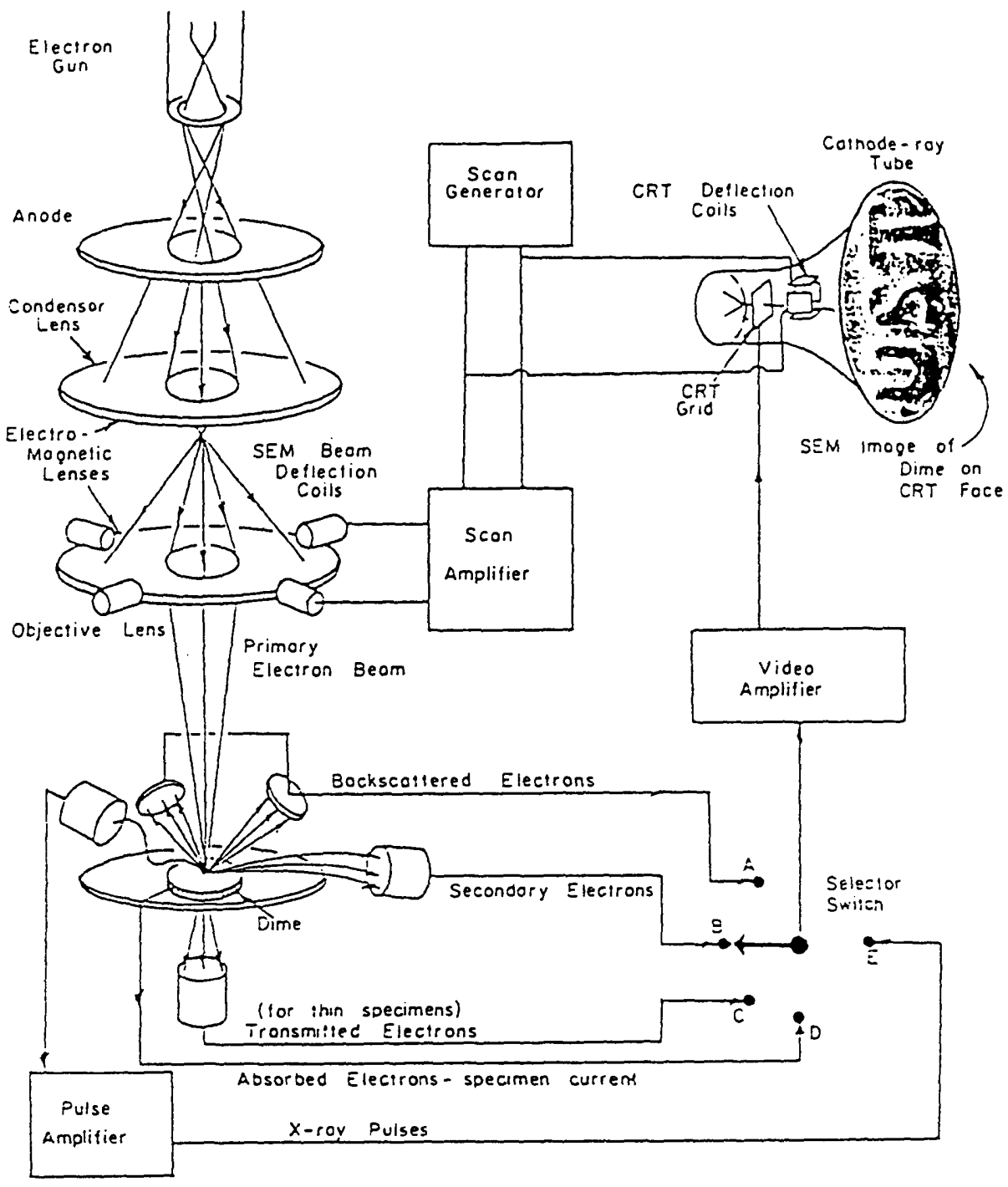

Figure 2.5-Schematic Diagram of a Scanning Electron Microscope (Ref. 38) 
vacuum chamber to collect the secondary or backscatter electrons and create a corresponding signal in a cathode ray tube. The deflection coils of the SEM's cathode ray tube are precisely synchronized with the coils controlling the electron probe deflection. A one-to-one correspondence therefore exists between the position of the probe on the specimen surface and the position of a spot on the cathode ray tube. The rectangular sweep of the probe over the specimen surface thus creates a complete image on the CRT screen ${ }^{38}$. A simplified schematic diagram of a scanning electron microscope is shown in Figure 2.5.

\subsubsection{Grain and Pore Size Measurements}

The grain size was measured in both the transverse and the longitudinal directions. A basic linear intercept methodology was used. This method was chosen over other methods based on its simplicity and the fact that most other methods assume equiaxed grains ${ }^{39}$. Although various correction factors are often used in conjunction with the linear intercept method ${ }^{40}$, it was decided that the results should be unfactored to reduce confusion and allow for a more effective comparison to grain size data from other sources. The grain size values obtained represent the length of a line drawn across the etched surface of the specimen divided by the number of grains intercepted by that line. This method was used for measurements in both the transverse and longitudinal directions.

An estimate of the average pore size and the range of pore sizes present in each sample was made from simple statistical compilations of individual pore measurements. These measurements were made through comparison with a scaling line on the SEM view screen. The size range of pores does not include the large pores (referred to as macropores in the results section) that were occasionally observed in many samples. 


\subsubsection{Investigation of Grain Substructure}

A more delicate etching process was used to investigate the existence of a substructure of low angle boundaries resulting from dendritic solidification. Because these low angle boundaries consist basically of an organized array of edge dislocations, a careful etch reveals them under high magnification on the SEM. In order to view the grain substructure in several samples the application time of the microetchant was significantly shortened.

\subsection{Texture Analysis}

A Rigaku RIU300 x-ray diffractometer was used to determine if GASAR specimens show a strong preferred orientation in the direction of the longitudinal axis. An $\mathrm{x}$-ray diffractometer uses monochromatic $\mathrm{x}$-radiation directed at the specimen surface from varying angles of incidence. A Bragg's condition of diffraction established at a particular angle of incidence identifies crystallographic orientation ${ }^{8}$. The basis of the Bragg's condition is presented in Figure 2.6. It describes the condition when two coherent (in phase) radiation waves at a certain angle of incidence remain in phase after reflecting off parallel planes of atoms in a unidirectional crystal lattice of orientation $<h k l>$. In Figure 2.6, this condition exists when the angle $\theta$ is such that the sum of the lengths SQ and QT are equal to a whole number, $\mathrm{n}$, of wavelengths ${ }^{8}$. If the interplanar spacing of atoms with respect to the crystallographic direction $<h k l>$ is $d_{h k l}$, and the $x$-ray wavelength is $\lambda$, then this condition can be summarized as

$$
n \lambda=S Q+Q T,
$$

or

$$
n \lambda=d_{h k l} \sin \theta+d_{h k l} \sin \theta=2 d_{h k l} \sin \theta
$$

which is known as Bragg's law. Bragg's law is a simple expression relating the x-ray wavelength and interatomic spacing to the angle of incidence and reflection of the 


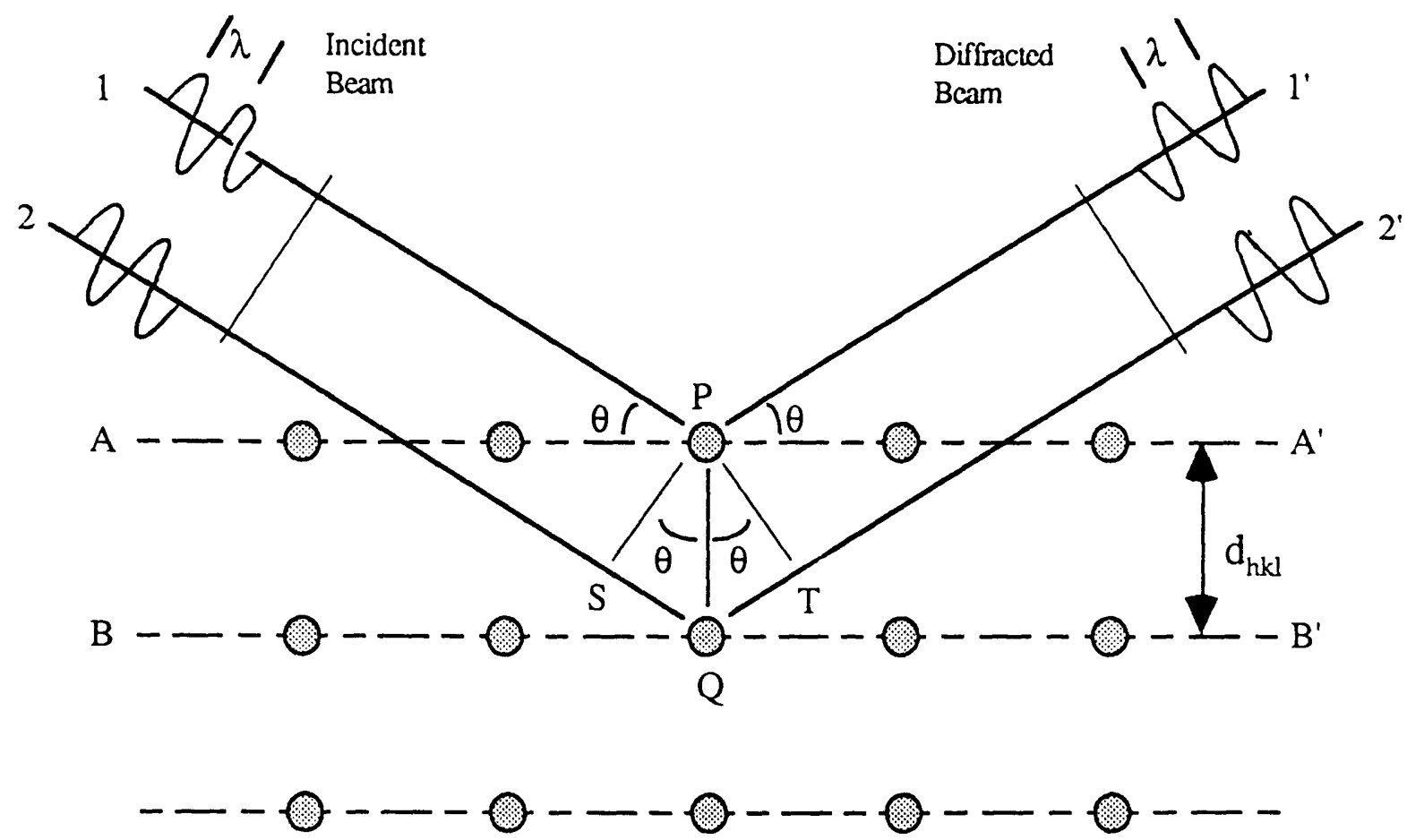

Figure 2.6 - Illustration of Bragg's Law for Diffraction

Monochromatic $\mathrm{x}$-rays are diffracted by atomic planes A-A' and B-B'. An angle $\theta$ that results in no change of relative phase in the reflected beam is a Bragg's angle. The satisfaction of Bragg's law is a necessary condition for diffraction. (Ref. 8)

diffracted beam. If the situation described by Bragg's law is not satisfied, destructive interference will occur in the diffracted beam and it will be incoherent. In materials with atoms placed in positions other than at the corners of the unit cell, however, interference can occur with

the diffracted beam for some planes. Bragg's law is thus a necessary but not completely sufficient condition for diffraction to occur. In FCC materials like copper, the result of the interference is that only directions with the Miller indices being either all even or all odd will be detected. Therefore, a diffractometer will show a $<200>$ plane in an FCC 
material, but not a $<100>$ plane, although the two planes have the same normal and indicate a similar crystallographic orientation.

For cubic base unit cell structures, like FCC, the interplanar spacing is a function of the Miller indices $\mathrm{h}, \mathrm{k}$, and $\mathrm{l}$, and of the lattice spacing parameter, $\mathrm{a}$, as follows:

$$
d_{n k l}=\frac{a}{\sqrt{h^{2}+k^{2}+l^{2}}}
$$

Using this relationship and the one established in Bragg's law, each angle of incidence at which diffraction occurs can be matched with a specific crystallographic orientation.

A schematic diagram of an x-ray diffractometer is shown in Figure 2.7. The diffractometer is configured to measure the angle at which diffraction of an $\mathrm{x}$-ray beam occurs. A flat polished surface of the specimen to be tested is initially aligned to be

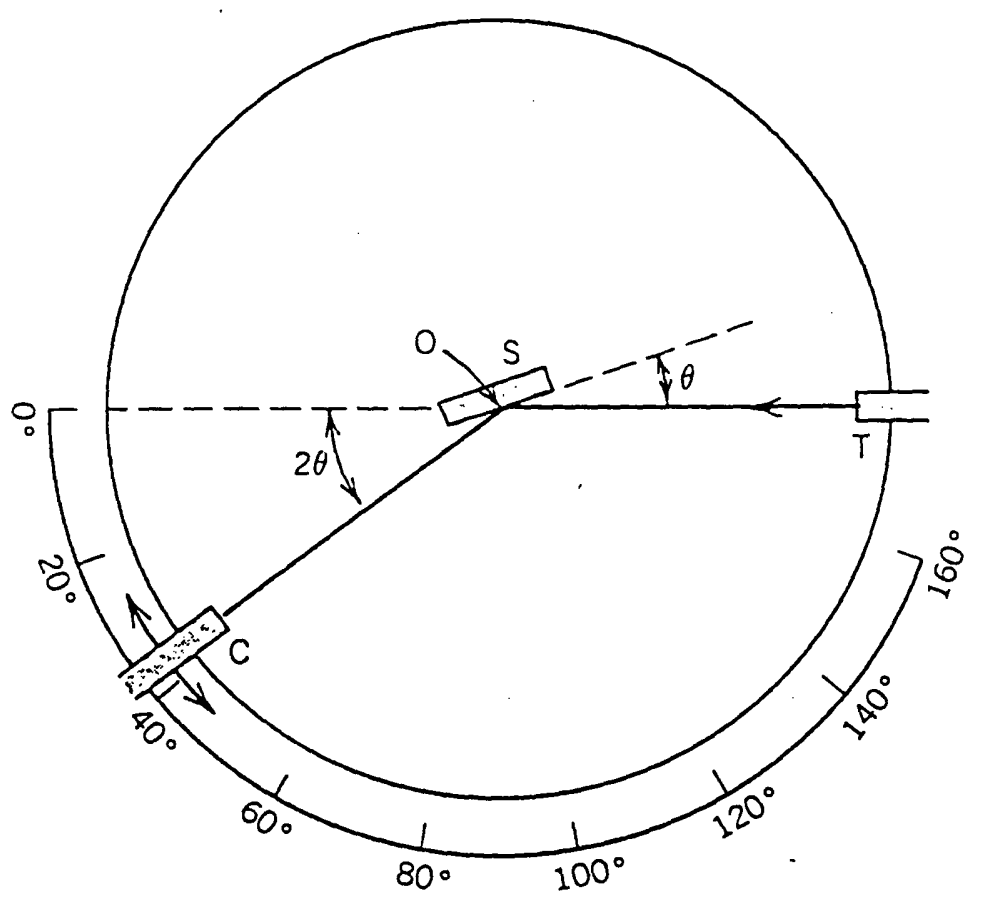

Figure 2.7 - Schematic of an X-ray Diffractometer

$\mathrm{T}=\mathrm{x}$-ray source, $\mathrm{S}=$ specimen, $\mathrm{C}=$ detector, and $\mathrm{O}=$ the axis about which the specimen and detector rotate. The result is a graph plotting the intensity of the detector signal with the angle $2 \theta$. (Ref. 8) 
coplanar with the x-ray beam. A counter which detects the intensity of the diffracted beam is also in line with the beam. As the specimen face is rotated to an angle $\theta$ with respect to the $x$-ray beam, the counter is rotated by $2 \theta$, such that the angle between the source and specimen face normal is equal to the angle between the specimen face normal and counter. The diffracted beam intensity is recorded and plotted as a function of $2 \theta$. Peaks on this plot are matched with crystallographic directions, and thus the crystallographic orientations of the grains causing diffraction are determined. Comparison of the relative height of the peaks appearing on this plot to the height distribution of peaks on a diffractometer plot of a material with randomly oriented grains indicates the existence of any preferred orientation. The extent of the preferred orientation can only be roughly determined. The x-ray diffractometer is thus an effective tool for determining texture in materials with strong preferred orientation, but not for providing a quantitative breakdown the orientations present within a sample.

\subsection{Chemical Analysis}

Chemical analysis data was obtained from two sources. An electron probe was first used to find the approximate purity of the sample. An electron probe operates in a fashion similar to the scanning electron microscope. It directs a concentrated electron beam at a sample and measures the resulting scatter of electrons. In the case of the electron probe, however, the energy level of the scattered electrons are recorded and a count made of the number of electrons measured at each electron energy level. Each element produces scattered electrons of different energy levels. By comparing the recorded count vs. energy level data to a series of standard plates for each atomic element, the elements present within a sample can be determined. The limitations of this method is that only elements which are present at a weight percentage of roughly $1 \%$ or 
more will be effectively detected. This preliminary method was used to verify that the GASAR samples were more than $99 \%$ pure copper.

Impurity data was also obtained from independent testing laboratories with optical emission and chemical analysis equipment capable of producing an exact breakdown of compositional elements ${ }^{41}$. This analysis was done to make a comparison of the impurity levels and impurity elements of both the nonporous and porous GASAR specimens. Any differences in impurity composition were recorded to determine if impurities could have a significant role in the strength of the porous materials. The fact that the porous specimens are solidified in a hydrogen atmosphere while the nonporous material is solidified in an argon atmosphere makes this comparison necessary. 


\section{Chapter Three : Presentation of Results}

The following chapter presents the results of the tensile testing, microscopic analysis, diffractometer testing, and chemical analysis performed on GASAR porous and nonporous specimens.

\subsection{Materials}

The measured porosity of the copper-based GASAR specimens which were received from DMI is plotted in Figure 2.1 for each nominal pore size. The porosity distribution for each pore size varied significantly from what was ordered. There was only a slight overlap in the porosity ranges of the nominal $50 \mu \mathrm{m}$ and $500 \mu \mathrm{m}$ pore size specimens. Additionally, the nominally nonporous specimens were found to have a porosity of approximately 0.035 . The pores in this material were found to be cylindrical and running diagonally through the ingot. These pores, which were approximately 0.5 $\mathrm{mm}-1 \mathrm{~mm}$ in diameter and $1 \mathrm{~cm}-4 \mathrm{~cm}$ in length, ran roughly from the edges of one end face to the center of the opposite end face in a cone-like configuration. Additional pores were dispersed through the ingot.

\subsection{Tensile Testing Results}

The following sections present data gained from the uniaxial tensile testing of the porous and nonporous specimens obtained from DMI. The machining and testing of the GASAR porous specimens was accomplished successfully, although the discontinuities caused by the pores necessitated frequent sharpening of the machining tools. The 


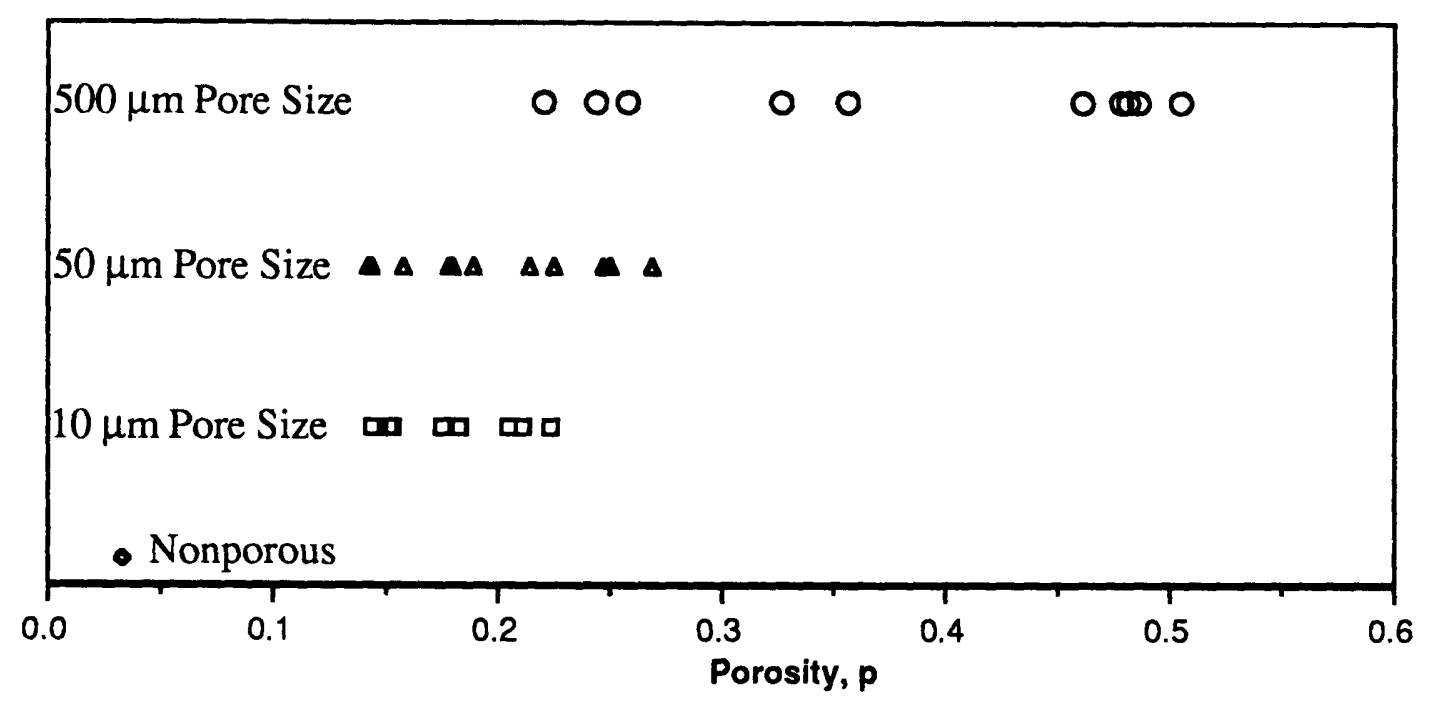

\section{Figure 3.1 - Distribution of GASAR Specimen Porosities}

The porosity distribution is shown for specimens of each nominal pore size received from DMI. Note the small overlap of the porosity range of the $500 \mu \mathrm{m}$ pore size specimens with that of the $50 \mu \mathrm{m}$ pore size specimens.

diagonal pores which were found in the nominally nonporous material caused serious problems in its testing. Due to the fact that the pores were large and widely dispersed, tensile specimens with a waisted diameter of $5 \mathrm{~mm}$ were able to be cut from the nonporous ingot without interference from the pores. Because of their larger crosssectional area, the $12.5 \mathrm{~mm}$ diameter specimens were all intersected by a number of pores running through their gage length. The size and frequency of these pores were enough to make the tensile test results questionable as a true representation of the solid material properties. As a result, the data documented in the following sections is the result of tests of $5 \mathrm{~mm}$ diameter specimens only. The complete stress-strain curve was obtained 
for each of these specimens. The $0.2 \%$ offset yield strength, the ultimate strength, Young's modulus and the strain at peak stress were measured from each stress strain curve.

\subsubsection{Stress Strain Curves}

The complete stress strain curves of tested samples are shown in Figures 3.2 3.5. Figure 3.2 shows the stress-strain curves obtained from the $5 \mathrm{~mm}$ diameter nonporous specimens. Figures 3.3 - 3.5 show the stress-strain curves obtained from the GASAR porous copper specimens, grouped according to the nominal pore size.

\subsubsection{Yield Strength}

The yield strength of each specimen was measured from the stress-strain curve using an $0.2 \%$ strain offset. The measured values are plotted in Figure 3.6 as a function of porosity. Figure 3.7 plots the net area yield strength versus porosity. With the exception of the most porous specimens, all measured yield strength values are significantly lower than the data provided by DMI. There is a significant amount of scatter in the measured data, particularly in the nominal $50 \mu \mathrm{m}$ and $10 \mu \mathrm{m}$ pore size specimens.

Figure 3.7 shows that the net area yield strength of the porous GASAR specimens is significantly higher than that of the nonporous specimens. An increase of as much as $50 \%$ is evident in a number of specimens with porosities between 0.14 and 0.23. Because of the large amount of scatter, a definite relationship between net area yield strength and porosity cannot be seen in the nominal $10 \mu \mathrm{m}$ and $50 \mu \mathrm{m}$ pore size specimens. In the nominal $500 \mu \mathrm{m}$ pore size specimens, the net area yield strength is not significantly dependent on porosity.

The nominal pore size does not appear to influence the yield strength of the $10 \mu \mathrm{m}$ and $50 \mu \mathrm{m}$ specimens. The nominal $500 \mu \mathrm{m}$ pore size specimens, however, show a slightly lower net area yield strength than the smaller pore size specimens. 


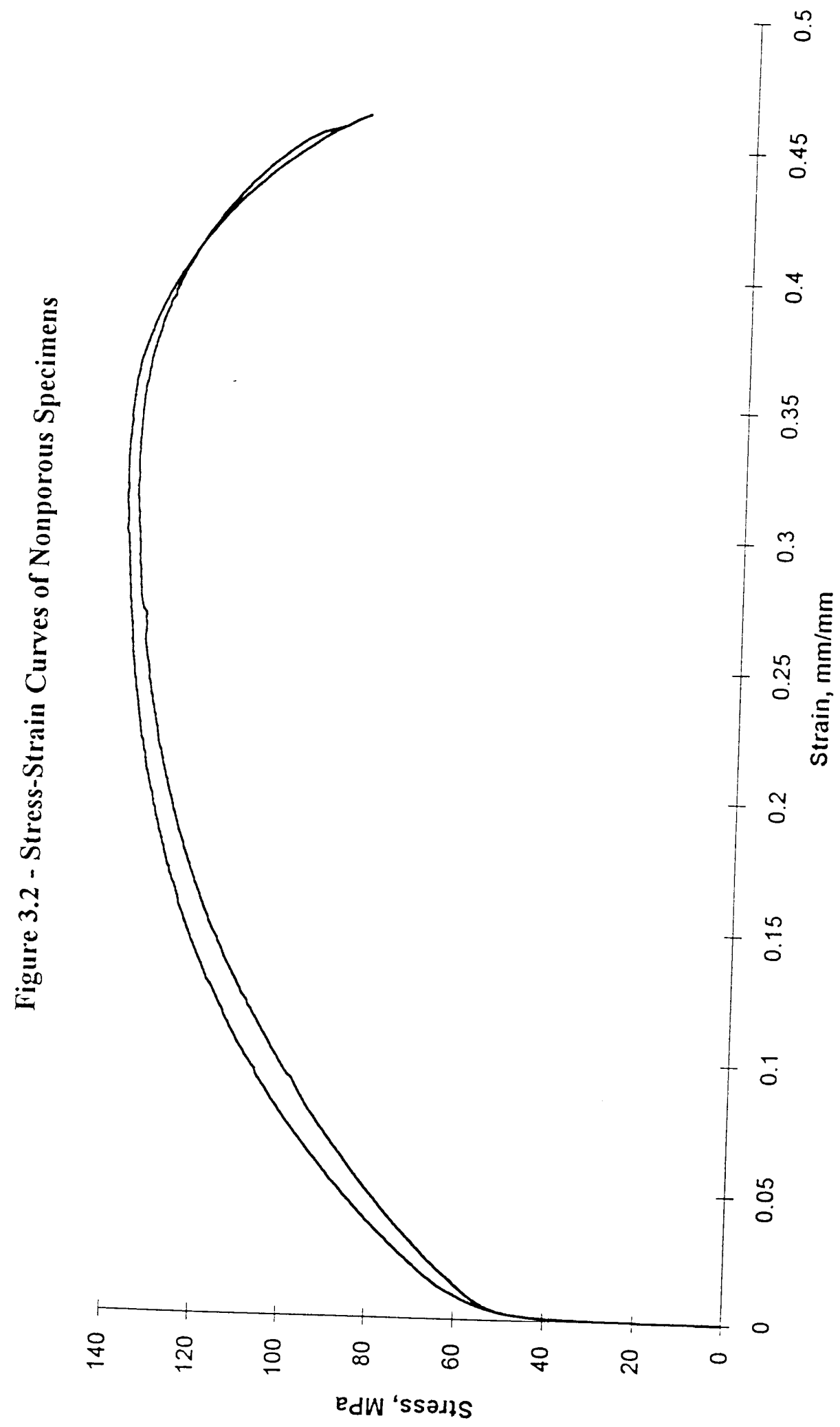




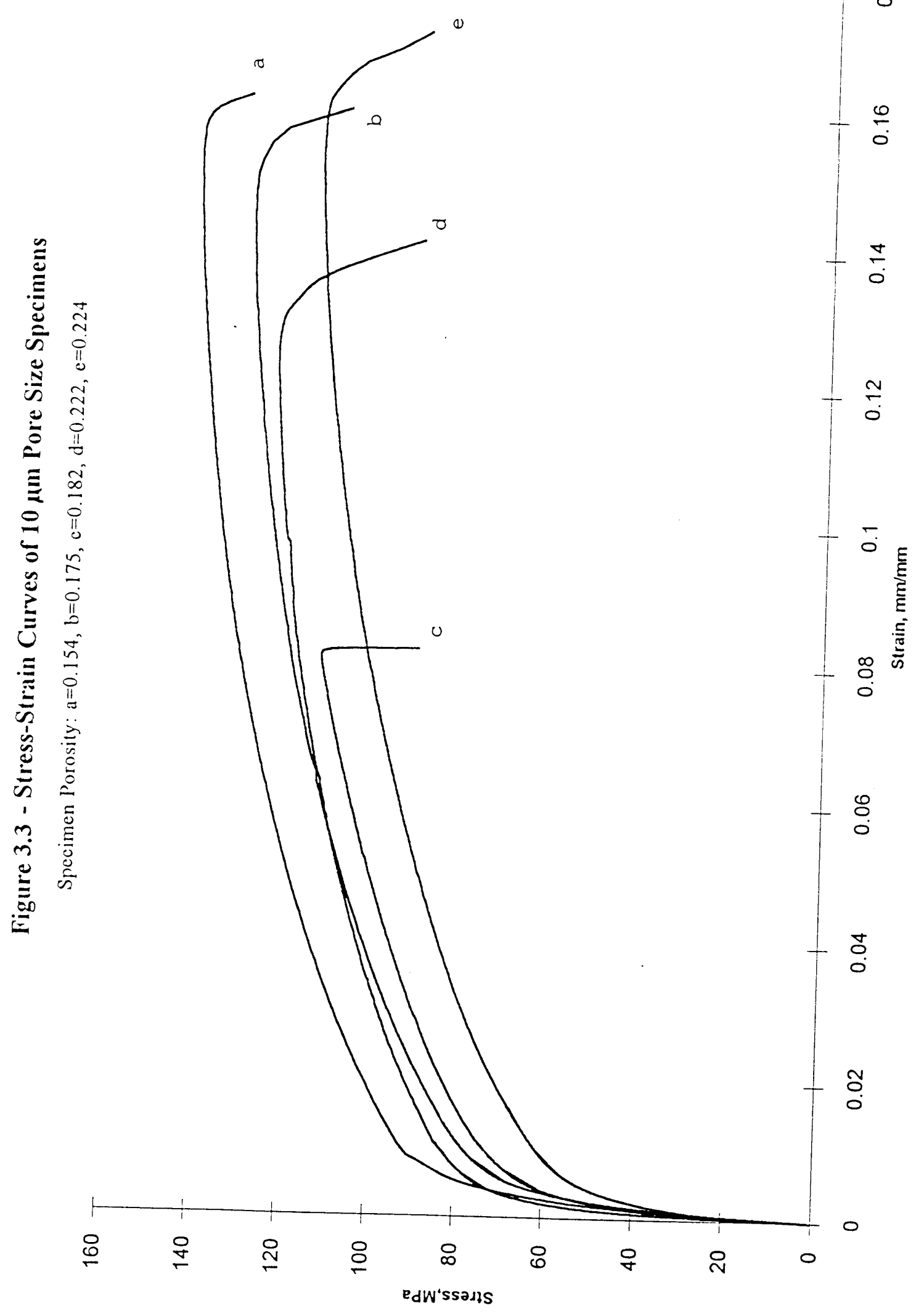

66 


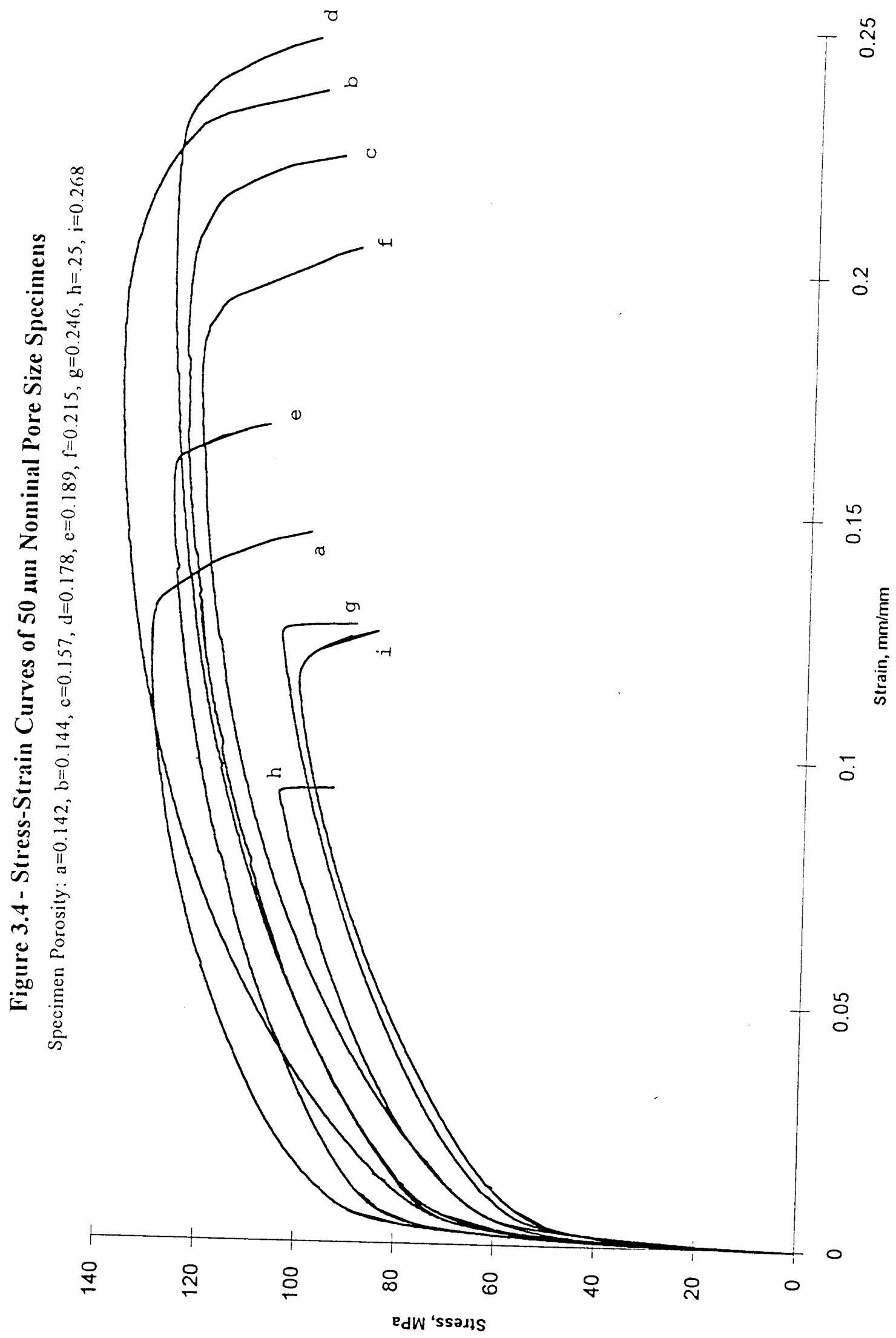




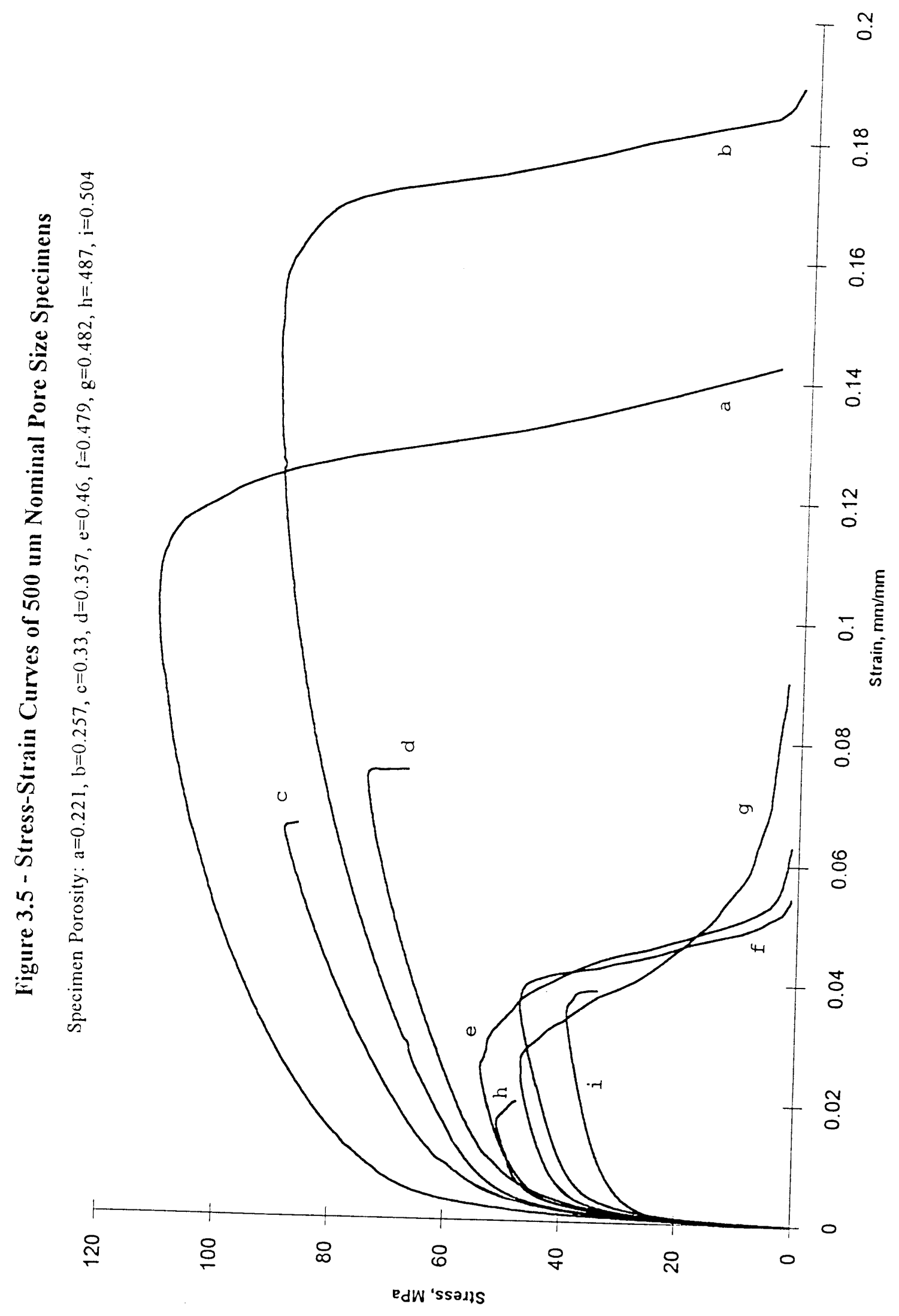




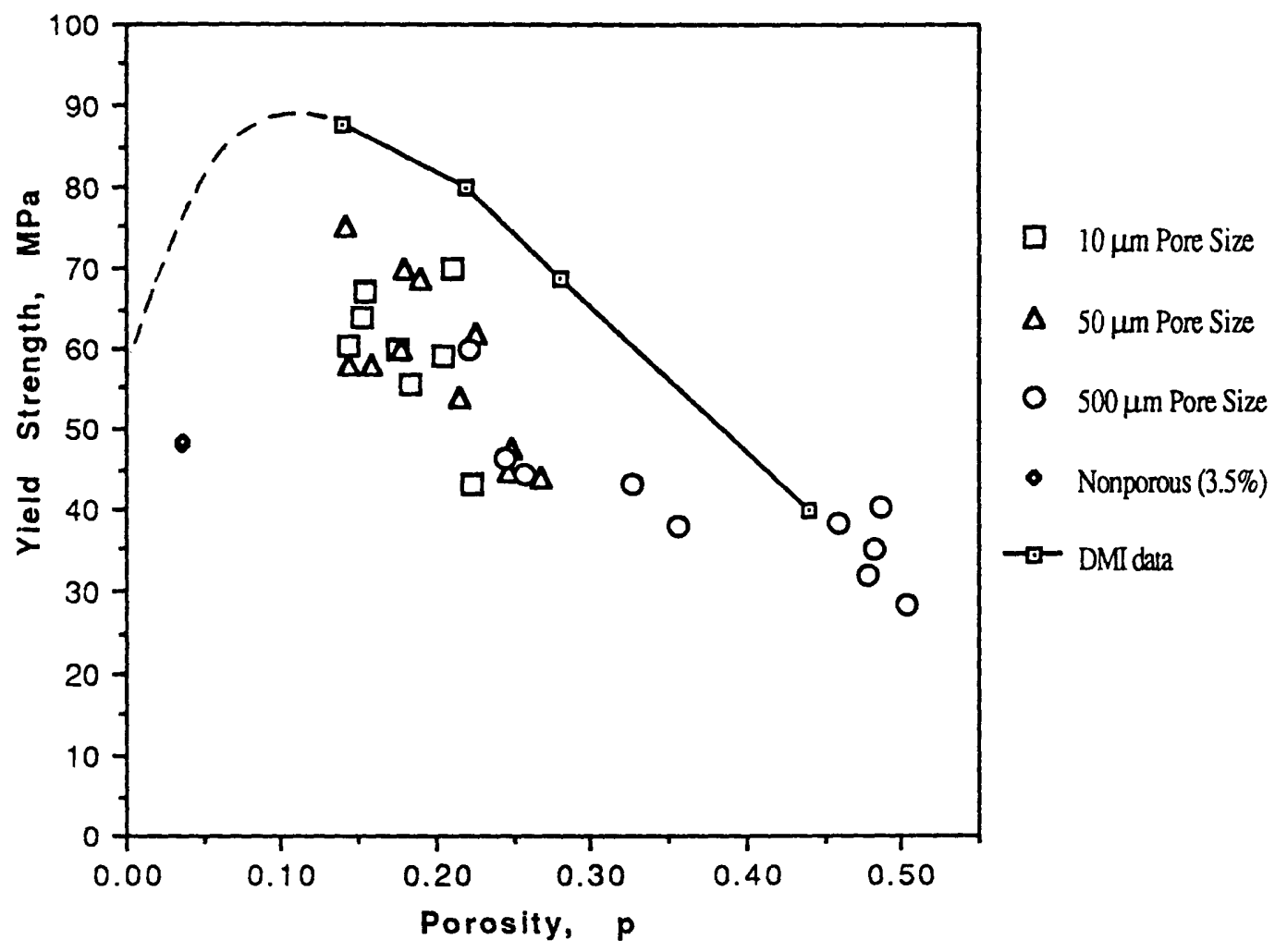

Figure $3.6-0.2 \%$ Yield Strength vs. Porosity Yield strength was measured using an offset strain of 0.002 . Method of DMI data measurement is unknown 


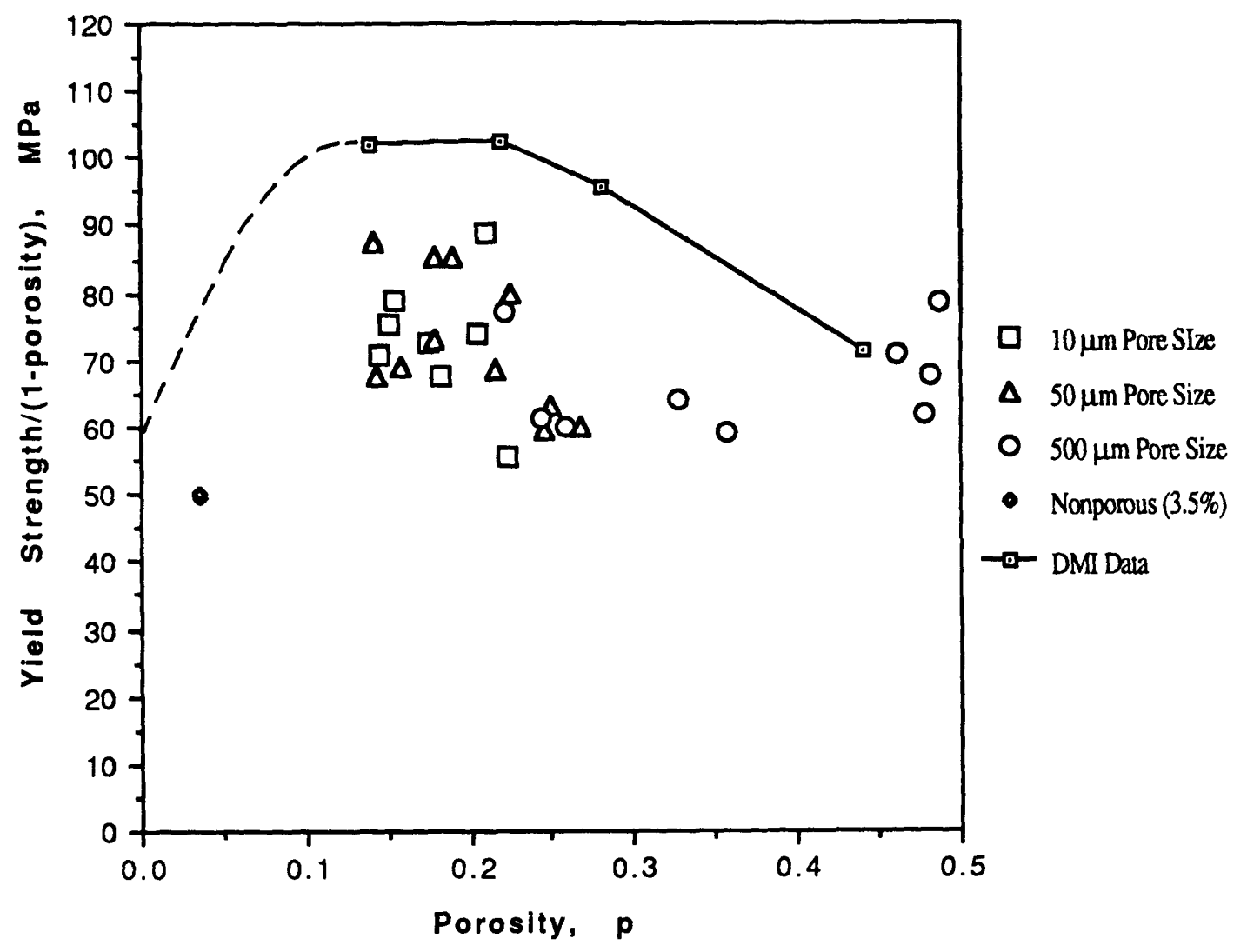

Figure 3.7 - Net Area 0.2\% Yield Strength vs. Porosity

The yield strength was measured using an offset strain of 0.002 . The method used in measuring the DMI data is unknown. 


\subsubsection{Ultimate Tensile Strength}

The ultimate tensile strength of each specimen was measured as the peak of its stress-strain curve. The measured ultimate tensile strength values are plotted versus porosity in Figure 3.8. The net area ultimate tensile strength is plotted versus porosity in Figure 3.9. The measured ultimate tensile strength values are only slightly lower than the DMI data, with significantly less scatter than that observed in the yield strength data.

Among the porous samples, the net area ultimate tensile strength decreases linearly with increasing porosity. A completely linear behavior for all porosities would indicate a nonporous material strength of $\approx 190 \mathrm{MPa}$. The measured ultimate tensile strength of the nonporous material was $\approx 142 \mathrm{MPa}$.

As with the yield strength data, there is no clear difference in data measured from the $10 \mu \mathrm{m}$ and $50 \mu \mathrm{m}$ nominal pore size samples. The relationship between strength and porosity seems to be independent of pore size.

\subsubsection{Young's Modulus}

The Young's modulus values measured from the stress-strain curves of the tensile specimens are plotted versus porosity in Figure 3.10. The net area modulus is plotted versus porosity in Figure 3.11. Because plastic deformation was found to begin at very low stresses in most specimens, the measured modulus values were taken from a small $(\varepsilon<0.001)$ portion of the stress-strain curve. The proportional limit of the curves was usually found to be less than $20 \mathrm{MPa}$.

From Figure 3.11, an interesting observation can be made. The data generally falls within one of two distinct linear bands. In the graph of solid area modulus vs. porosity, these bands are fairly horizontal. The upper band (with only 6 points) lies between $107 \mathrm{GPa}$ and $120 \mathrm{GPa}$, and the lower band (which contains the majority of the data points) lies between $50 \mathrm{GPa}$ and $80 \mathrm{GPa}$. Only the modulus of one nonporous 


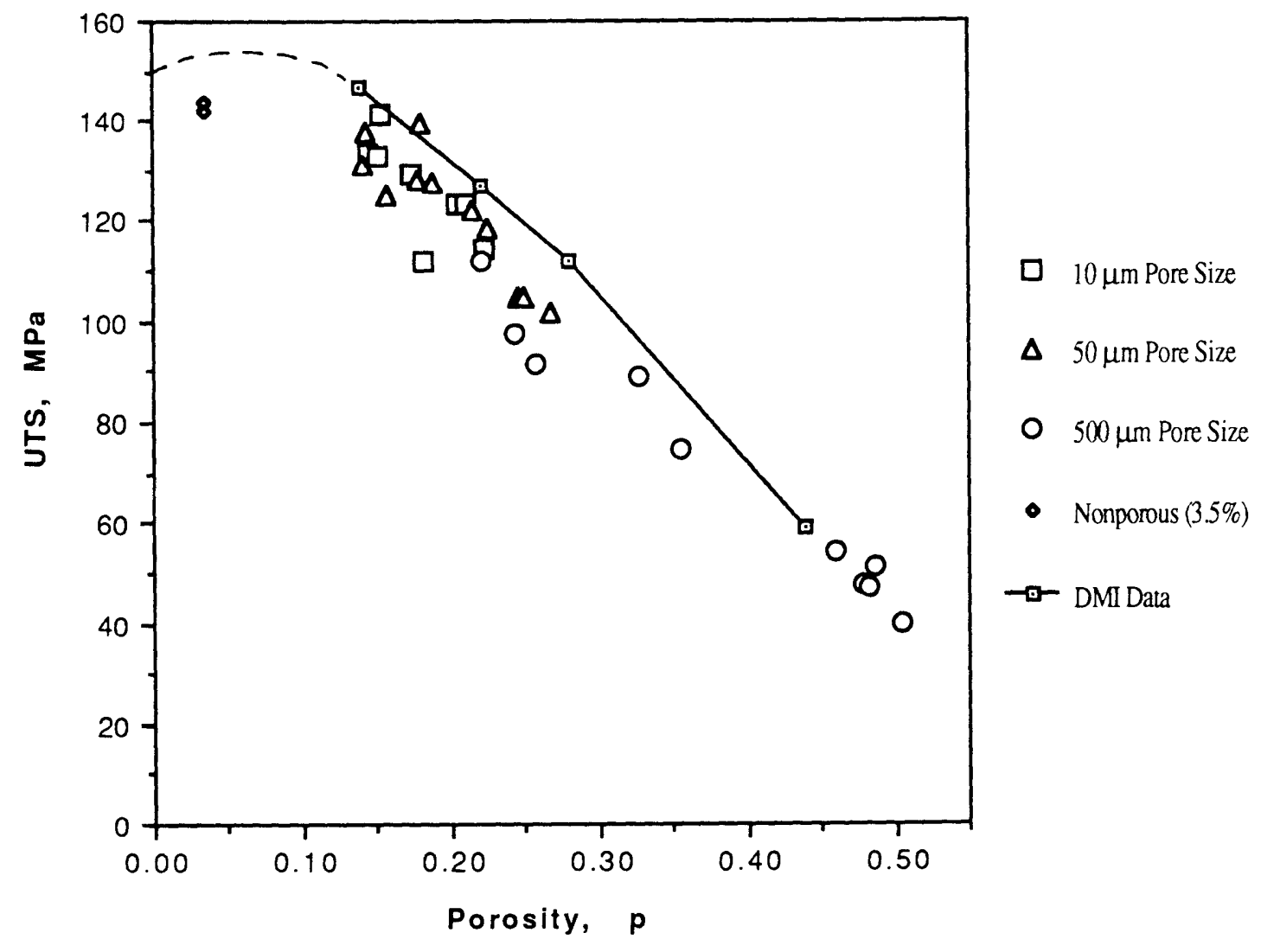

Figure 3.8 - Ultimate Tensile Strength vs. Porosity 


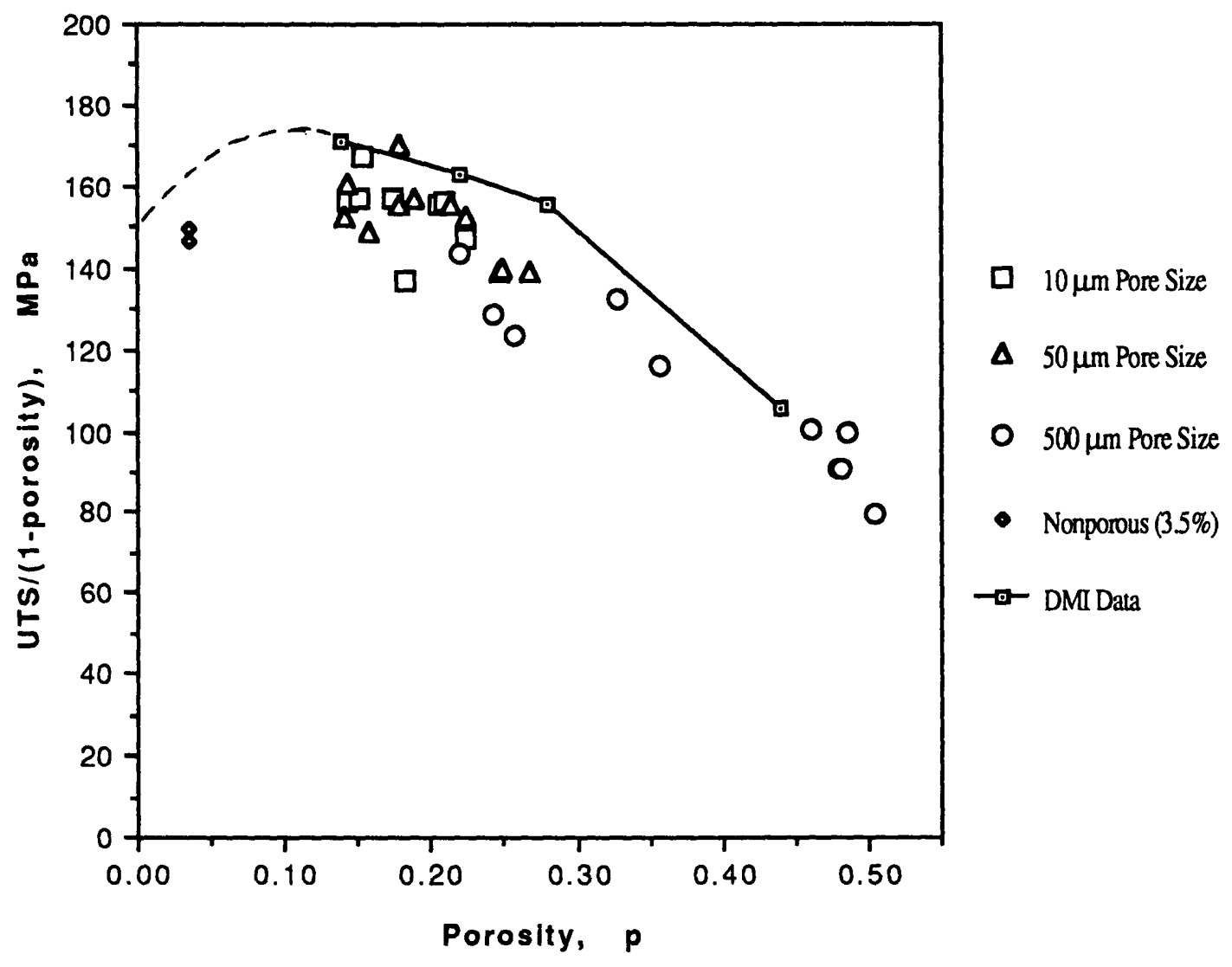

Figure 3.9 - Net Area Ultimate Tensile Strength vs. Porosity 


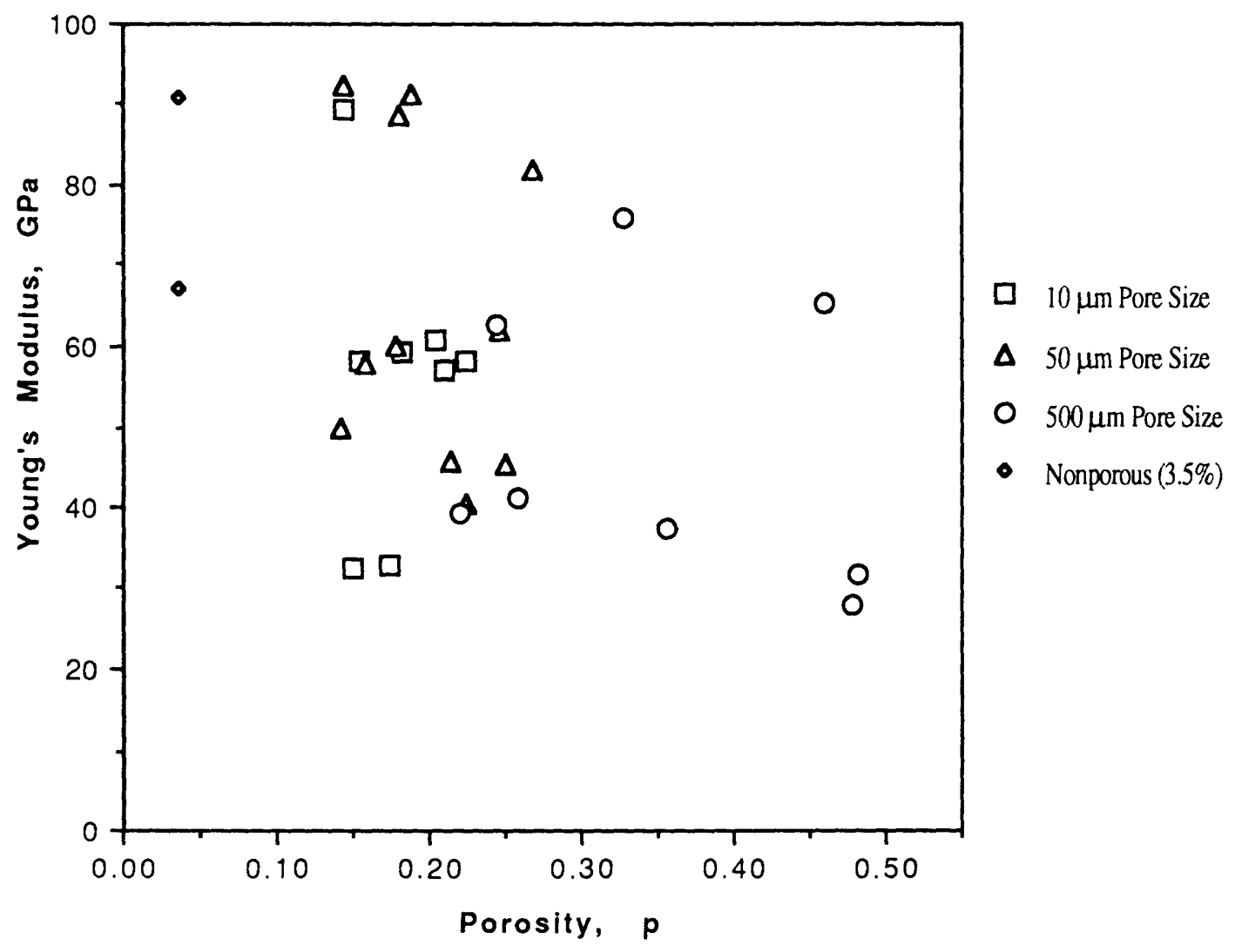

Figure 3.10 - Young's Modulus vs. Porosity 


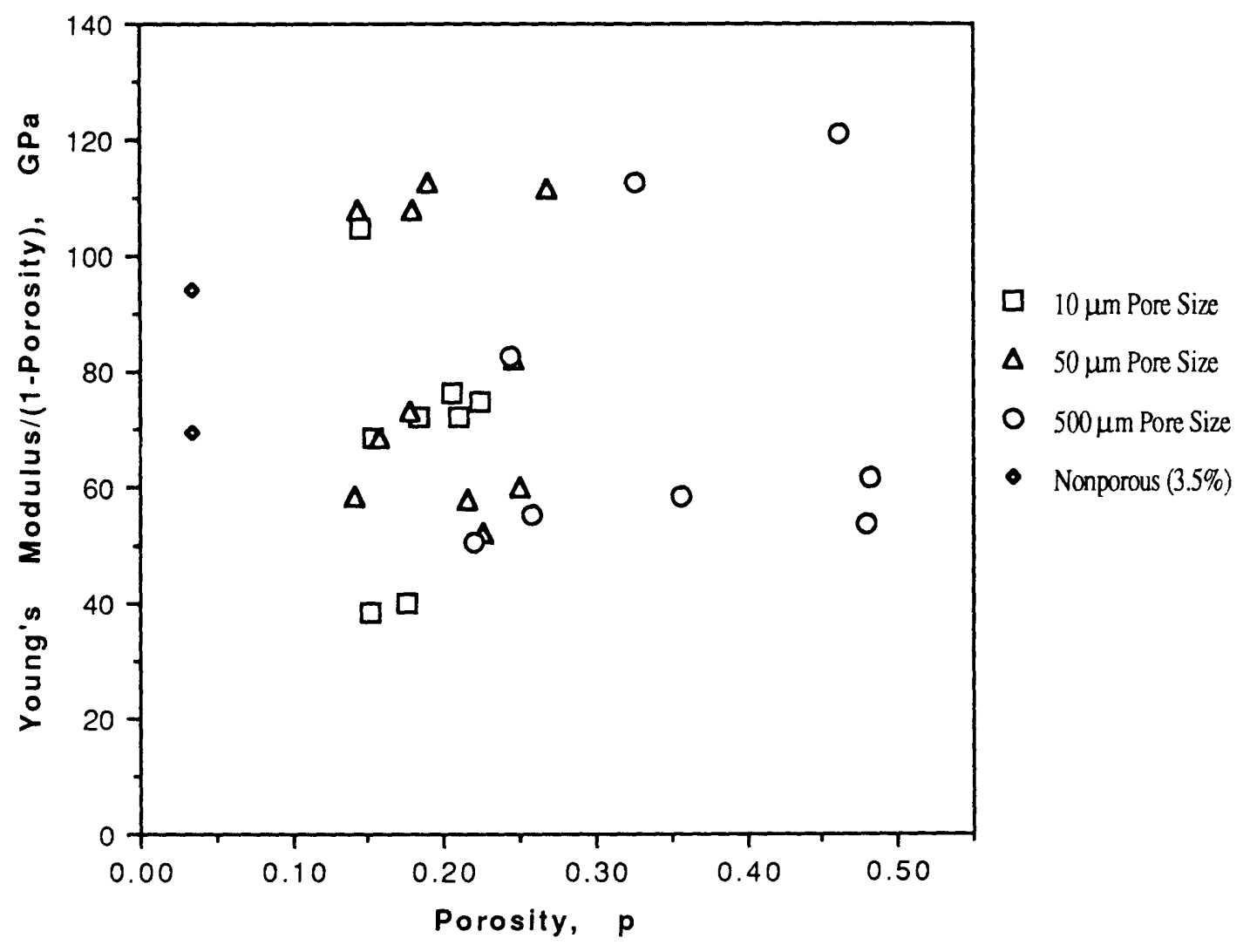

Figure 3.11 - Net Area Young's Modulus vs. Porosity 
specimen falls between these two distinct bands. Each of the bands of modulus values are independent of porosity.

\subsubsection{Modes of Fracture}

From observation of the curves in Figures $3.1-3.3$, it can be seen that the strain softening behavior of the GASAR porous specimens is inconsistent. While most specimens exhibited only a limited amount of strain softening before fracturing, several specimens, particularly those with a nominal $500 \mu \mathrm{m}$ pore size, showed extensive strain softening. This extensive softening was due to the stable propagation of either one or two tearing cracks across the specimen diameter. Because this tearing shifted the neutral axis of the specimen diameter, a bending deformation accompanied tear propagation.

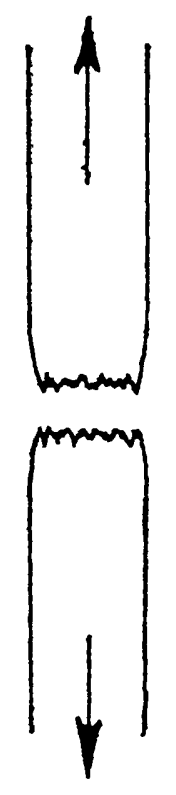

a

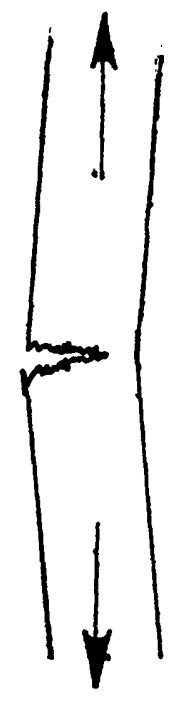

b

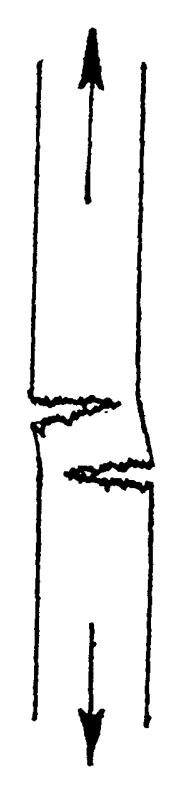

c

Figure 3.12 - Variation in Modes of Fracture

GASAR porous specimens were found to fracture in one of three ways: a) catastrophic brittle fracture, b) progressive tear propagation, or c) multi-tear propagation. 


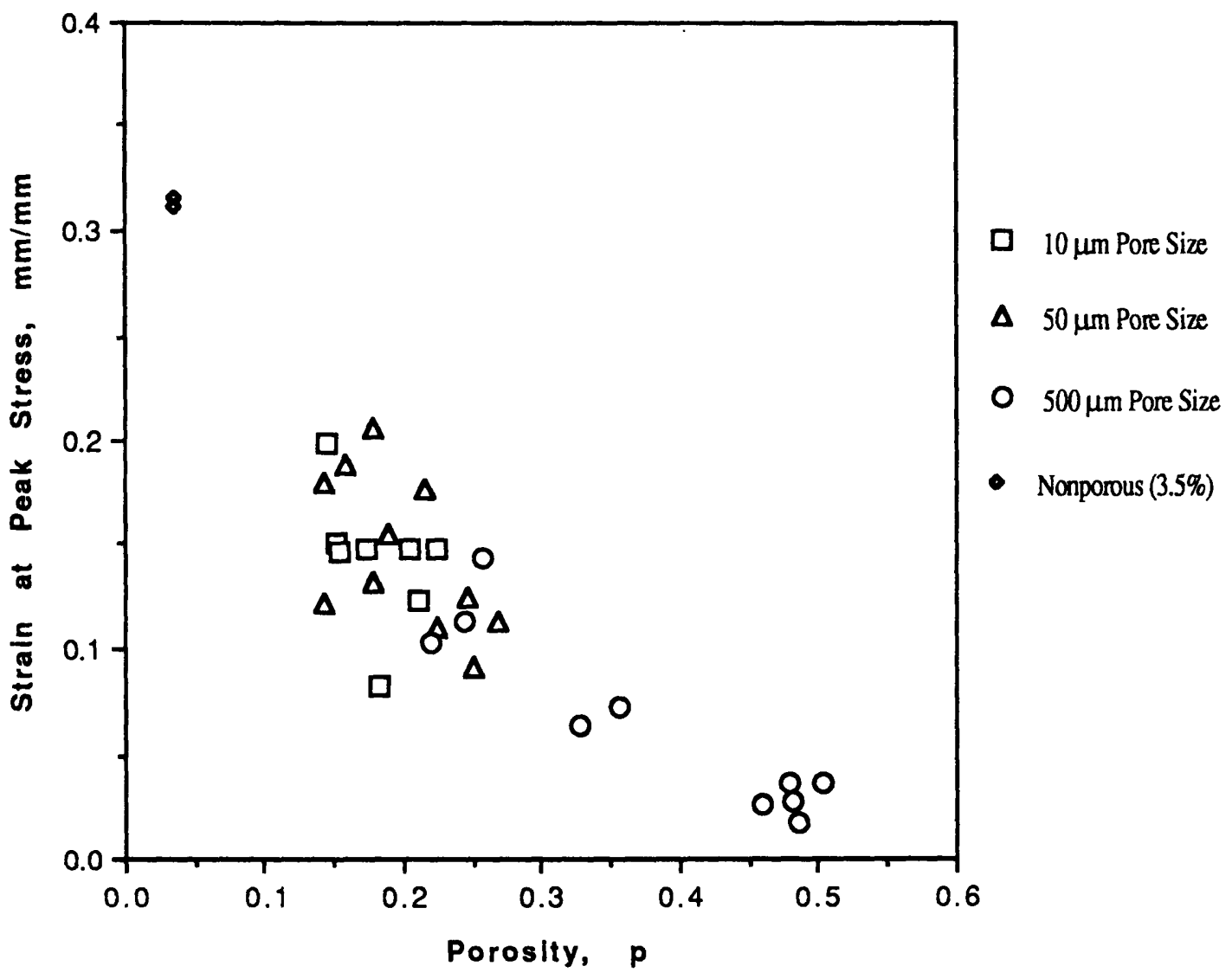

Figure 3.13 - Strain at Peak Stress vs. Porosity

Data represents the strain at which the ultimate tensile stress was recorded. 
Because of the bending, the strain measurement was dependent on the position of the extensometer with respect to the bending axis. Because the ultimate strain measured from the stress-strain curves of these specimens did not necessarily represent the true ductility of the specimen, the strain at which the ultimate tensile strength was measured was used as a measure of ductility. The different modes of fracture that were observed during testing are shown in Figure 3.12.

\subsubsection{Strain at Peak Stress}

The strain at peak stress was recorded from each stress-strain curve as a measure of the specimen's ductility The results of these measurements are plotted vs. porosity in Figure 3.12. It can be seen that the ductility is highly dependent on porosity. The strain at peak stress for the nonporous sample was much higher than that measured for the porous specimens. The peak stress occurs in the nonporous specimens at a strain of $\approx 0.33$. The ultimate strain of $\approx 0.45$ (see Figure 3.2 ) of the nonporous sample is comparable with the reference value of annealed pure copper ${ }^{4}$. In the porous specimens, the ductility decreases non-linearly with increasing porosity. The ductility data does not show a significant dependence on the nominal pore size.

\subsection{Cross-Sectional Micrographs}

This section presents sample electron micrographs (SEM) of the type used for grain measurements and microstructural characterization. Both transverse (perpendicular to the testing direction) and longitudinal (parallel to the testing direction) are presented. Two micrographs are shown of both longitudinal and transverse cross sections of specimens from each nominal pore size group. The two micrographs represent the lower and upper bounds of porosity range of each nominal pore size.

Figures 3.14, 3.15, and 3.16 show transverse cross-sectional views of $10 \mu \mathrm{m}$, $50 \mu \mathrm{m}$, and $500 \mu \mathrm{m}$ nominal pore size specimens, respectively. Note the change in scale in micrographs of the $500 \mu \mathrm{m}$ pore size specimens. At lower porosities, the pores of the 
$10 \mu \mathrm{m}$ and $50 \mu \mathrm{m}$ pore size specimens appeared in a ring-like network. The diameter of the grains is much larger than that of the pores; a single grain may contain several rings of pores. As porosity increases, a more dispersed pore structure can be seen. The nominal $500 \mu \mathrm{m}$ pore size specimens exhibit a much larger distribution of pore size than the smaller pore size specimens. In the cross-sectional micrographs, it is evident that the pores are separated by solid walls, indicating a closed cell material.

Figures 3.17, 3.18, and 3.19 show longitudinal views of $10 \mu \mathrm{m}, 50 \mu \mathrm{m}$, and $500 \mu \mathrm{m}$ nominal pore size specimens, respectively. Note the change in scale of the 500 $\mu \mathrm{m}$ pore size specimens. The grain boundaries are much more difficult to see in these views due to the orientation of the pores. As expected, the pores are much longer in the longitudinal direction than in the transverse direction. These views also indicate a closed cell pore structure.

\subsection{Fracture Surface Micrographs}

Sample micrographs of the fracture surface of porous GASAR specimens are shown in Figure 3.20. Compare these micrographs with the transverse cross-sectional micrographs of undeformed specimens with the same nominal pore size (Figures 3.14, 3.15 , and 3.16). Note that the pores on the fracture surface are roughly the same size, or perhaps slightly larger than those in the undeformed specimen. Also note that the solid material undergoes localized area reduction which forms ridges between the pores at fracture. The reduction in area of the total cross-sectional area is much smaller than that of the net cross-sectional area. Close inspection of these micrographs shows signs of straining in directions perpendicular to the loading direction. 
Figure 3.14 - Transverse Cross-Section Micrographs of $10 \mu \mathrm{m}$ Nominal Pore Size Specimens

a) Porosity $=0.151$, b) Porosity $=0.222$

a)

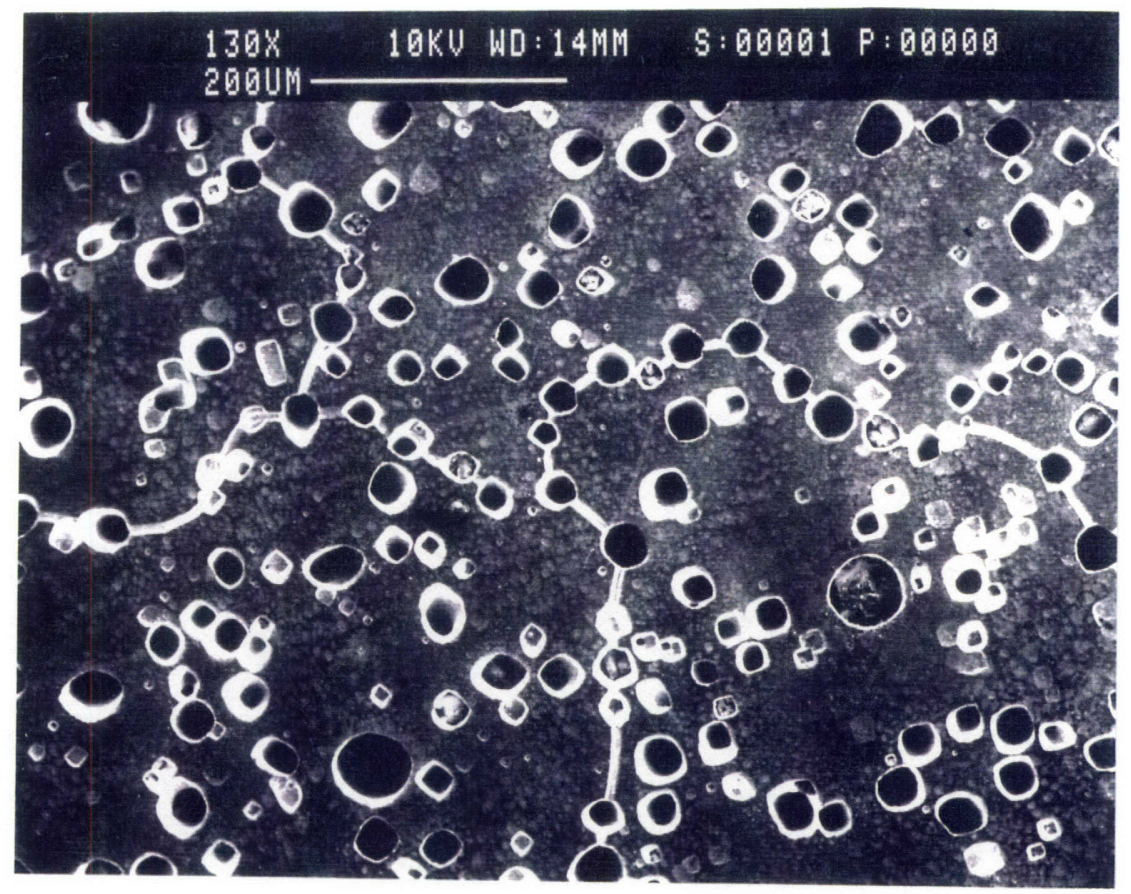

b)

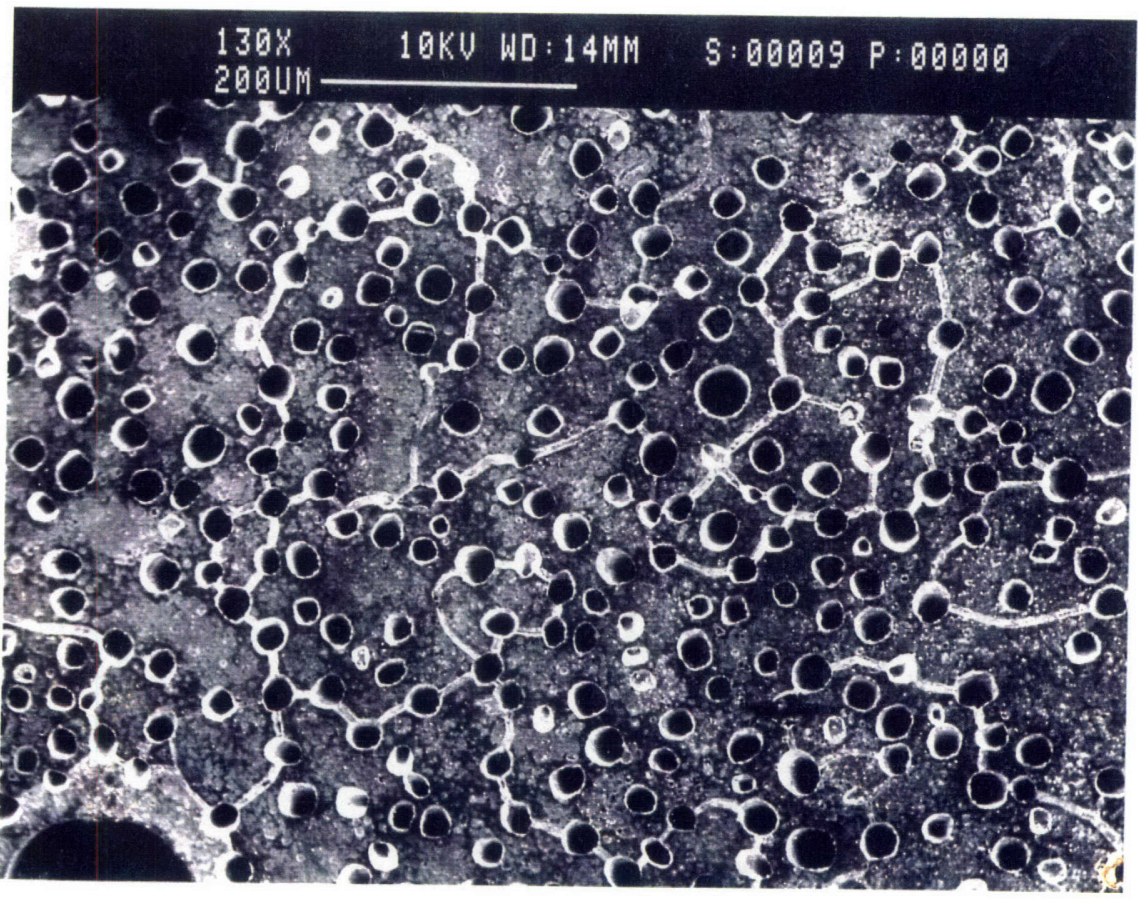


Figure 3.15 - Transverse Cross-Section Micrographs of $50 \mu \mathrm{m}$ Nominal Pore Size Specimens

a) Porosity $=0.144$, b) Porosity $=0.225$
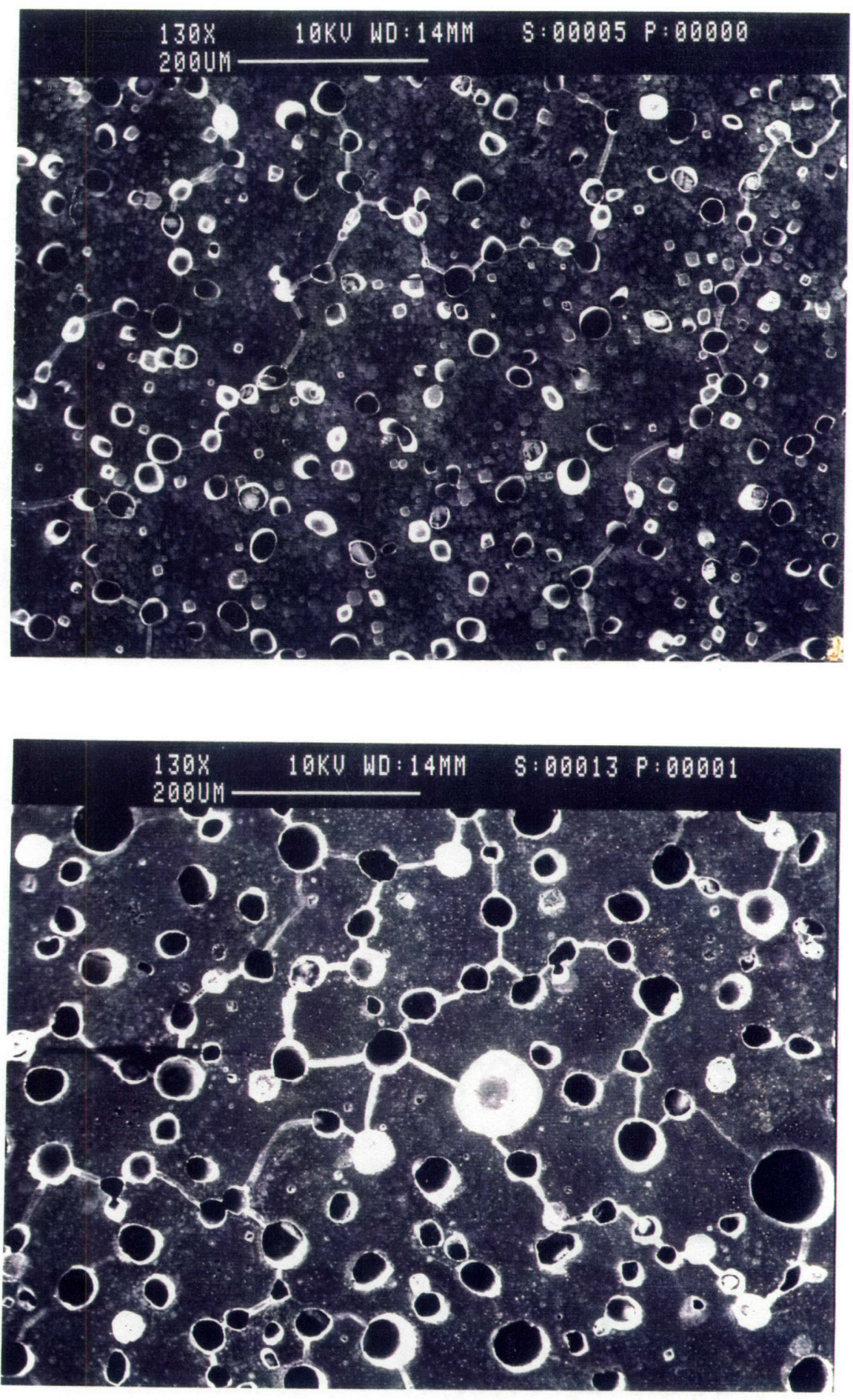
Figure 3.16 - Transverse Cross-Section Micrographs of 500 $\mu \mathrm{m}$ Nominal Pore Size Specimens

a) Porosity $=0.221$, b) Porosity $=0.478$

a)

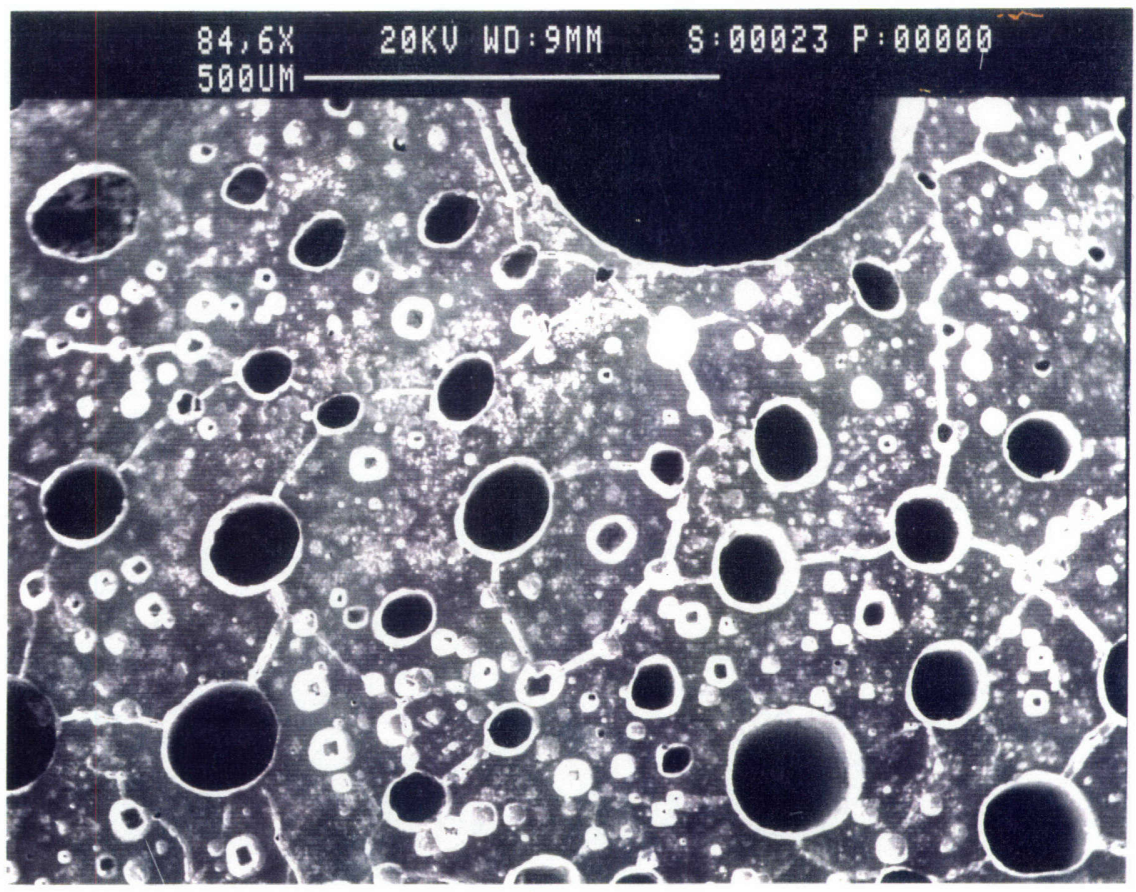

b)

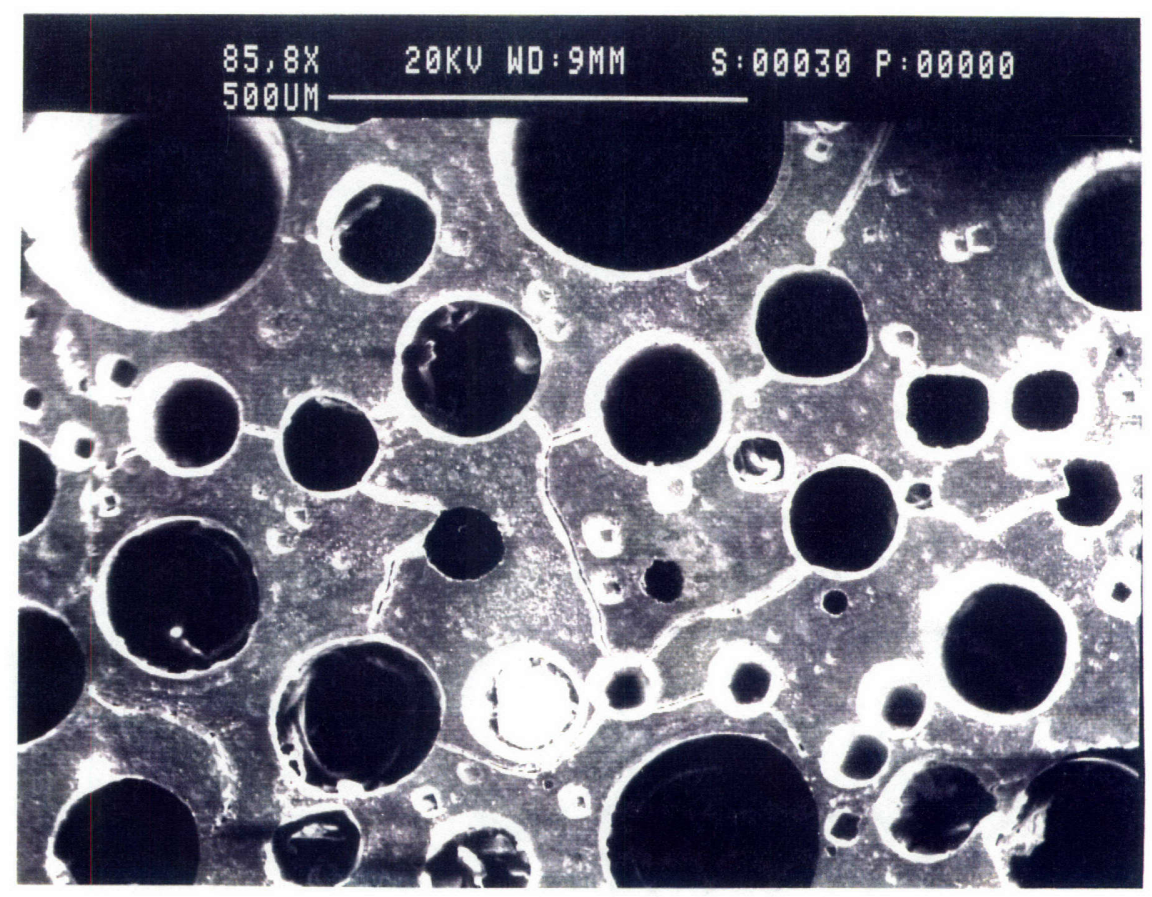


Figure 3.17 - Longitudinal Cross-Section Micrographs of 10 $\mu \mathrm{m}$ Nominal Pore Size Specimens

a) Porosity $=0.154$, b) Porosity $=0.224$

a)
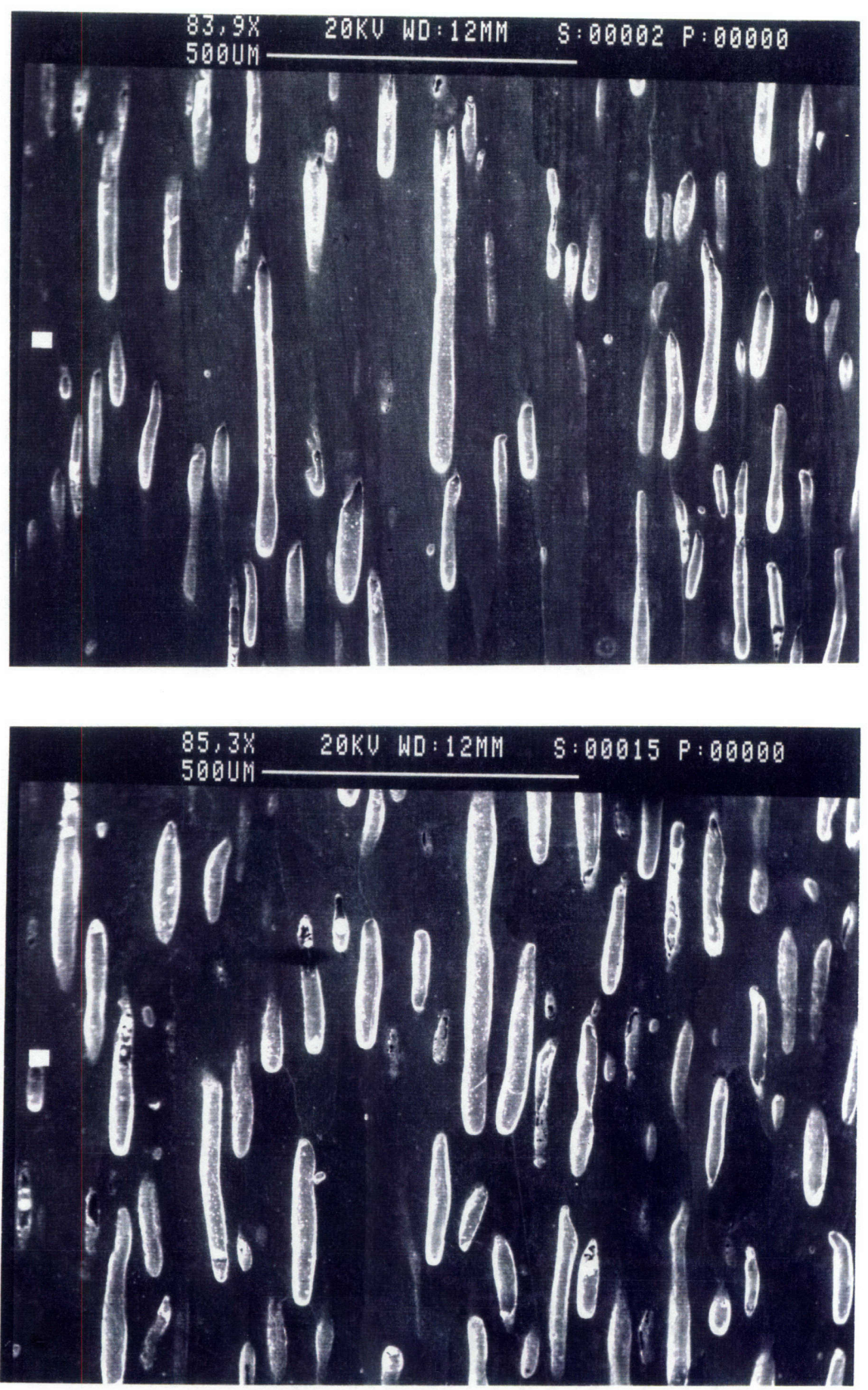

b) 
Figure 3.18 - Longitudinal Cross-Section Micrographs of 50 $\mu \mathrm{m}$ Nominal Pore Size Specimens

a) Porosity $=0.0 .144, \quad$ b) Porosity $=0.0 .268$

a)

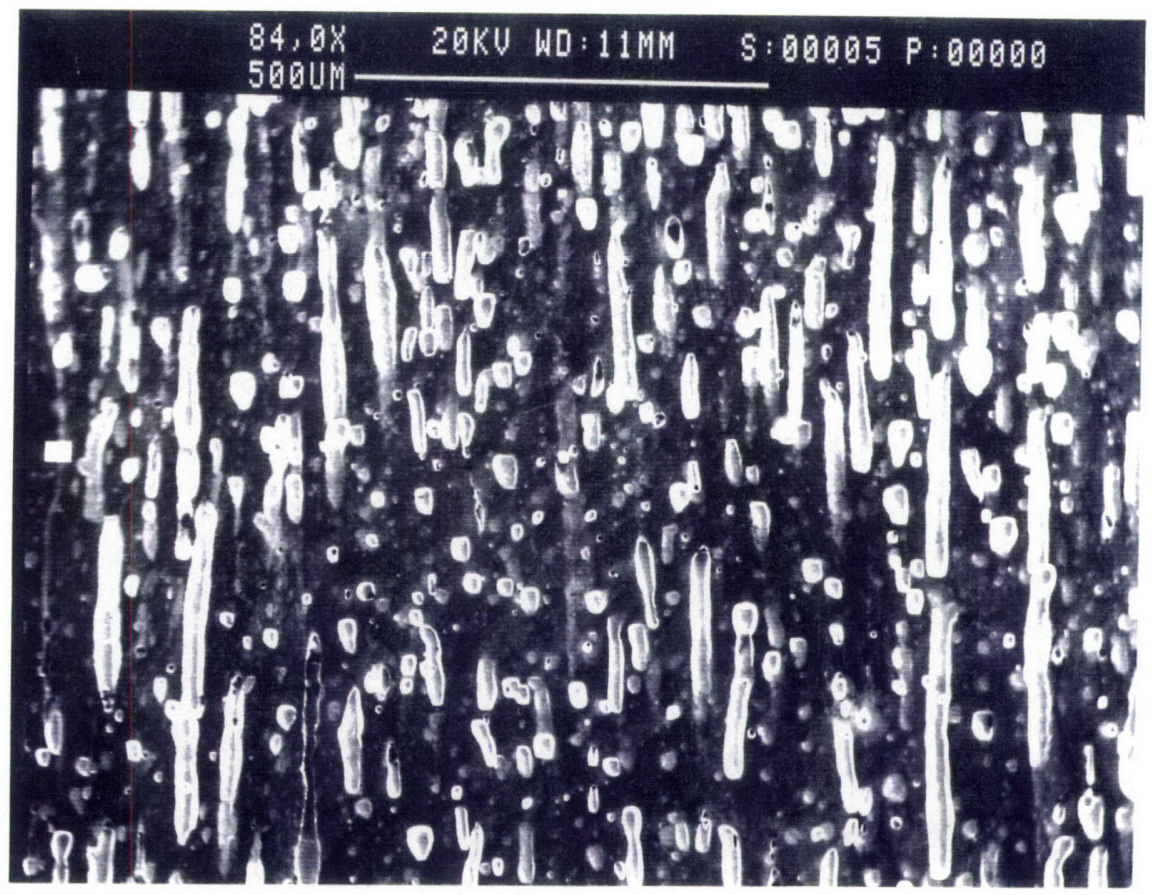

b)

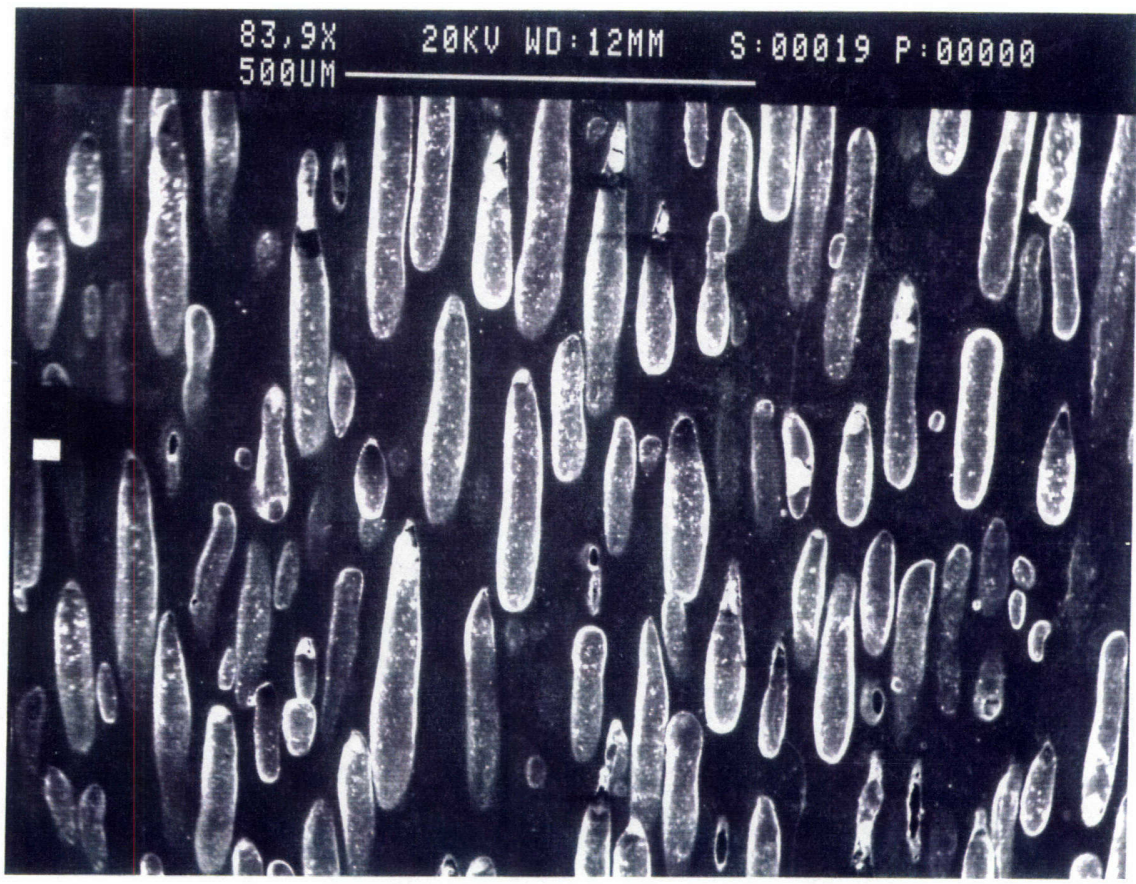


Figure 3.19 - Longitudinal Cross-Section Micrographs of 500 $\mu \mathrm{m}$ Nominal Pore Size Specimens

a) Porosity $=0.221$, b) Porosity $=0.479$

a)

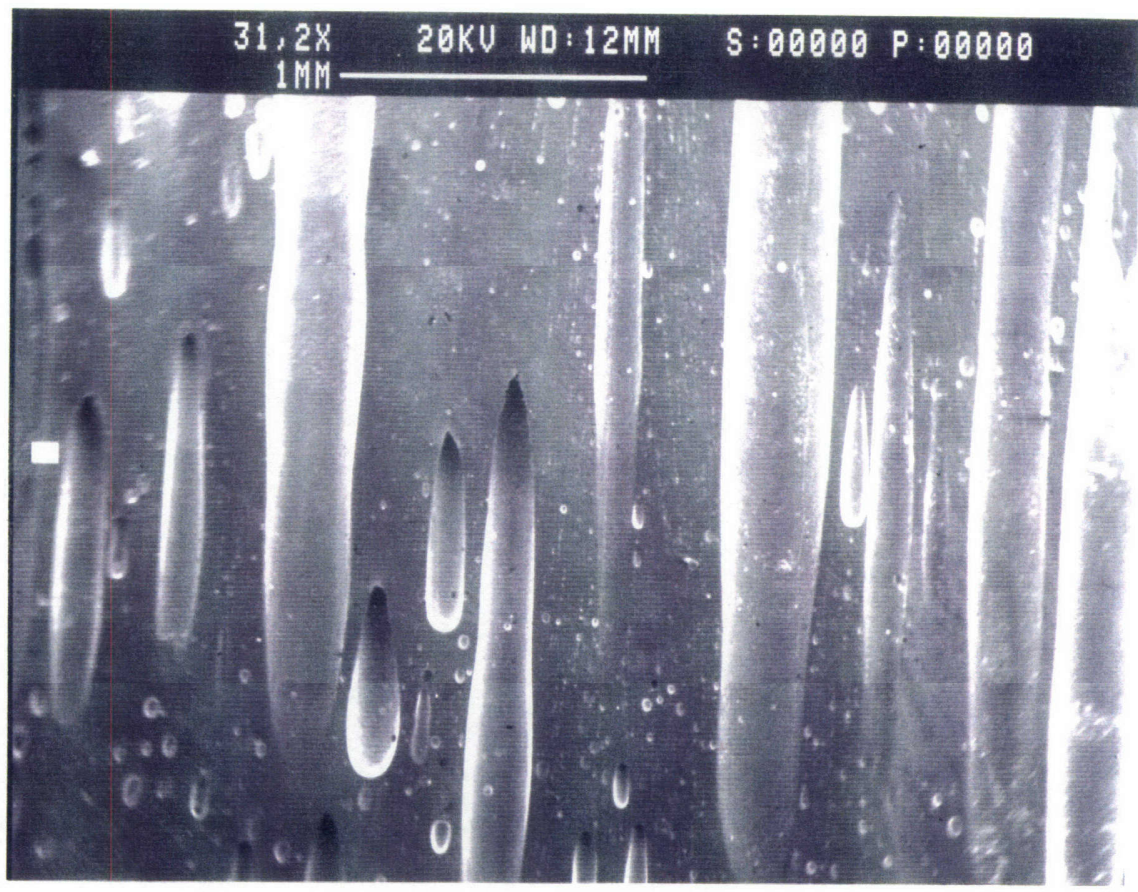

b)

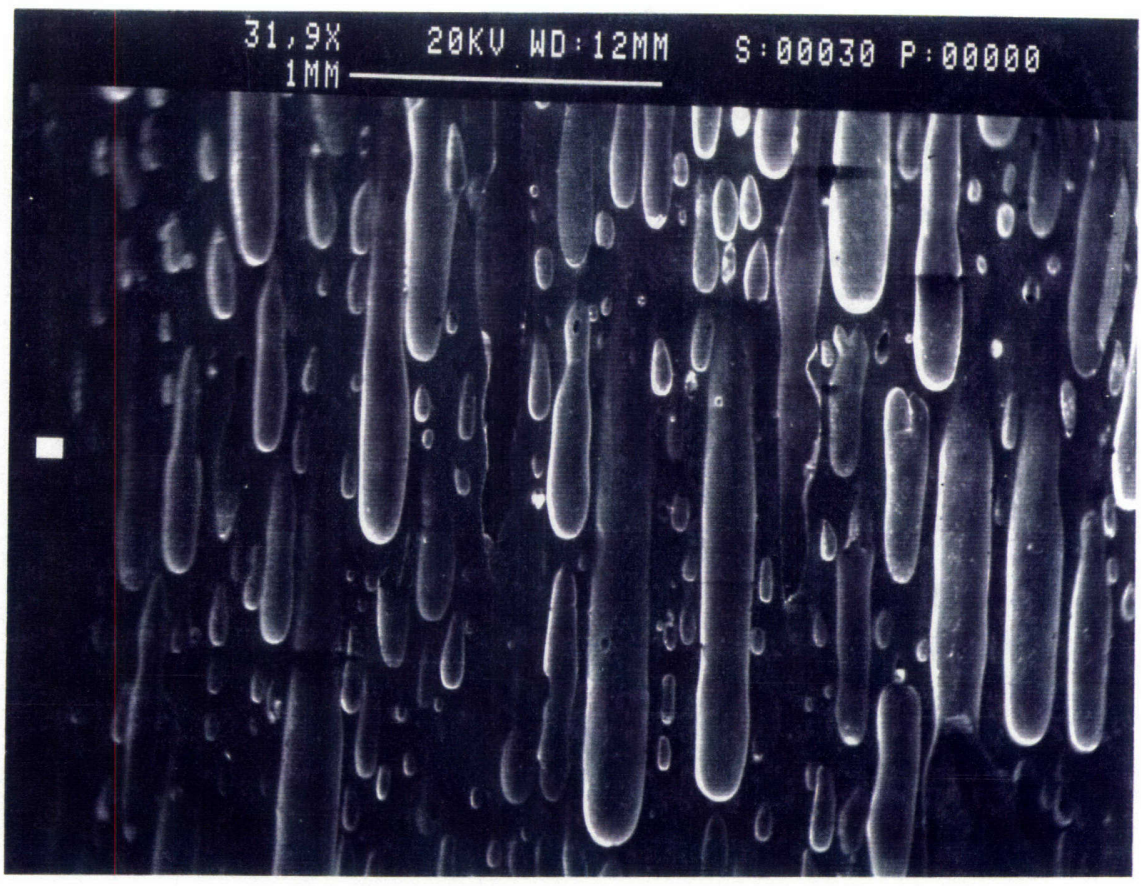


Figure 3.20 - Fracture Surface Micrographs

a), b) $10 \mu \mathrm{m}$ Nominal Pore Size, Porosity $=0.154$, c) $50 \mu \mathrm{m}$ Nominal Pore Size,

Porosity $=0.144$, d) $500 \mu \mathrm{m}$ Nominal Pore Size, Porosity $=0.479$
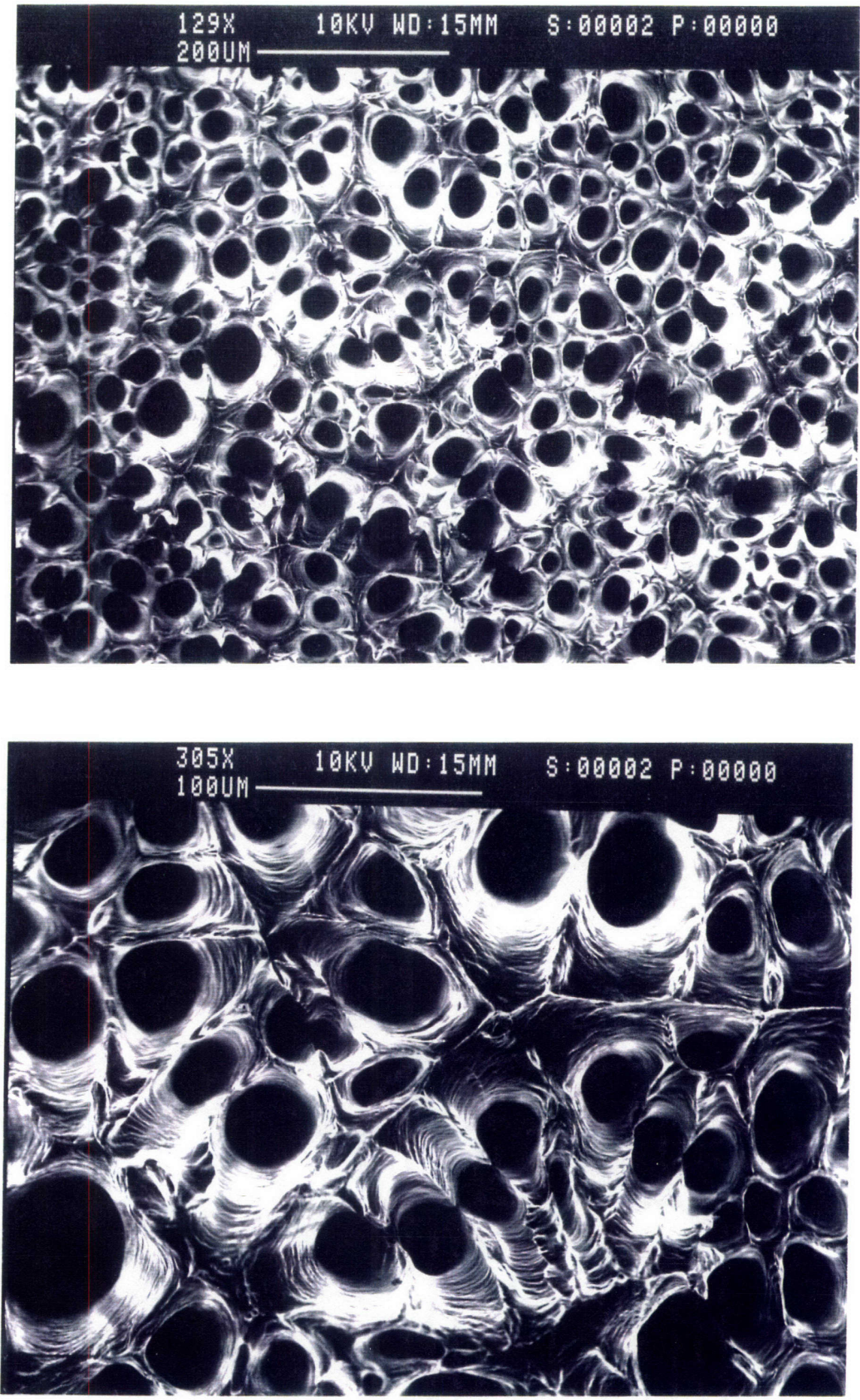
Figure 3.20 - Fracture Surface Micrographs (continued)

c)

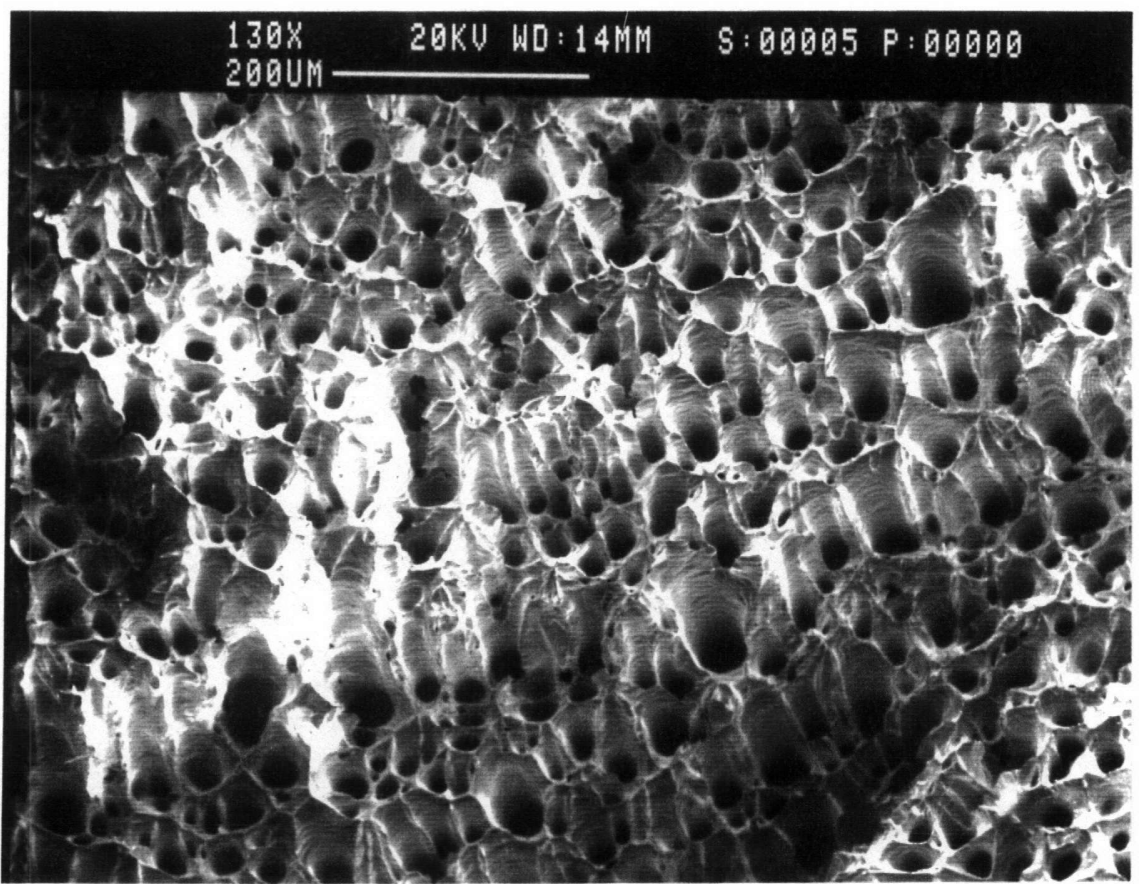

d)

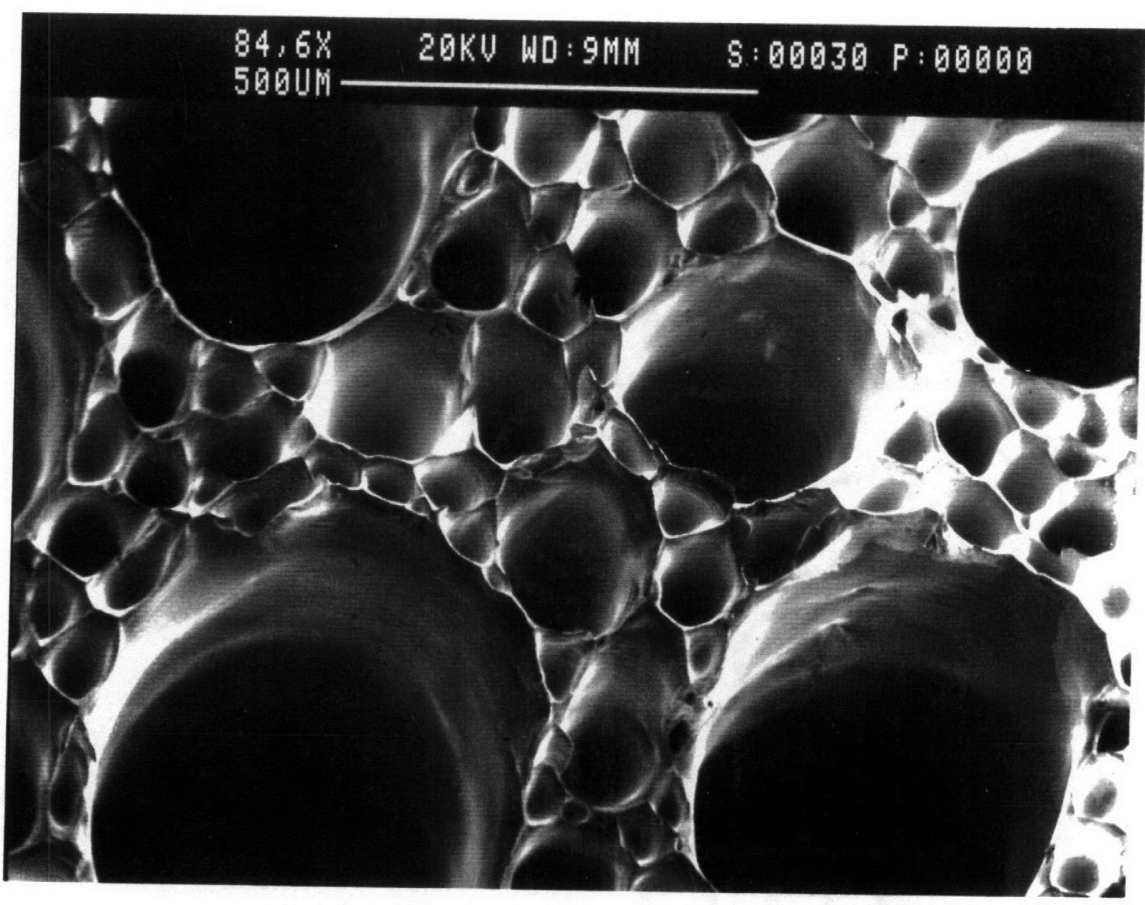




\subsection{Grain Measurements}

This section presents the results of the linear intercept grain size measurements made in both the transverse and longitudinal directions. No conversion factors are used with the measurements.

\subsubsection{Transverse Grain Size}

The transverse grain size measurements are shown as a function of porosity in Figure 3.21. It can be seen that both the porosity and pore size strongly influence the grain size. The grains are very large as a result of the casting process. The nonporous specimens contain much larger grains than any of the porous specimens. In the $10 \mu \mathrm{m}$ and $50 \mu \mathrm{m}$ nominal pore size specimens, the grain size decreased sharply with increasing porosity, in a fairly linear fashion. The grains in the $50 \mu \mathrm{m}$ pore specimens were slightly larger than the $10 \mu \mathrm{m}$ pore size specimens. All $500 \mu \mathrm{m}$ nominal pore size specimens had grains between $400 \mu \mathrm{m}$ and $600 \mu \mathrm{m}$, with a slight increase in size with increasing porosity.

\subsubsection{Longitudinal Grain Size}

Due to the difficulty in separating pore boundaries and grain boundaries, the longitudinal grain size measurements are subject to a much higher degree of error. The measurements were also limited by the length of the grains in the longitudinal direction. Many of the microscopy specimens did not contain enough grains to allow for an effective linear intercept measurement. For this reason, many samples were not used for these measurements, although an adequate number of samples were used to allow for a distribution of porosities and pore sizes. The results of the longitudinal grain size measurements are shown in Figure 3.22

The grains were generally much longer in the longitudinal direction than in the transverse. The grains are columnar and oriented parallel to the pore orientation. The relationship of grain length to porosity and pore size is similar to that of the transverse 


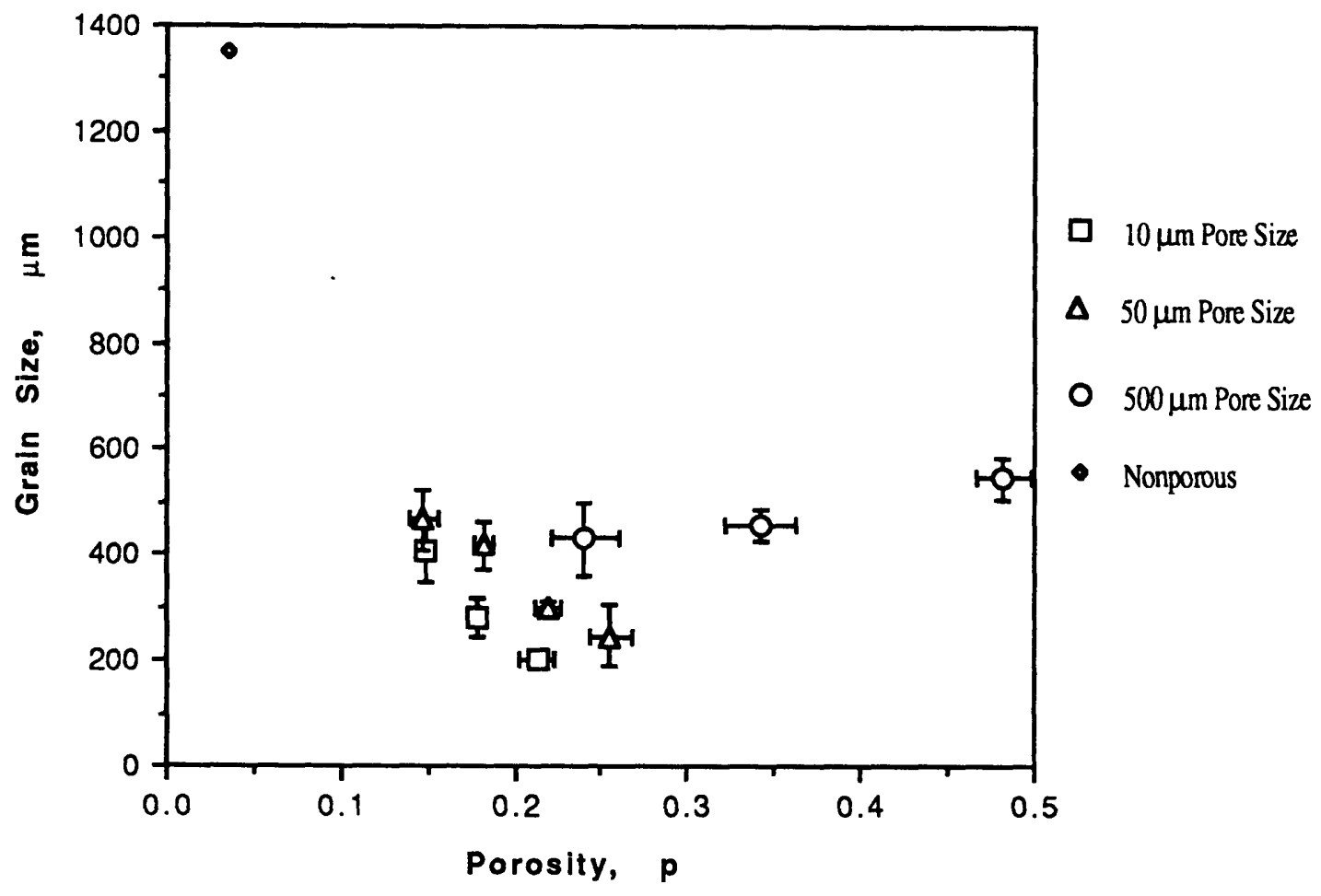

Figure 3.21 - Transverse Grain Size vs. Porosity

Data was measured using the line-intercept method. Each data point represents 2-5 specimens. The error bars indicate the standard deviation of the recorded data 


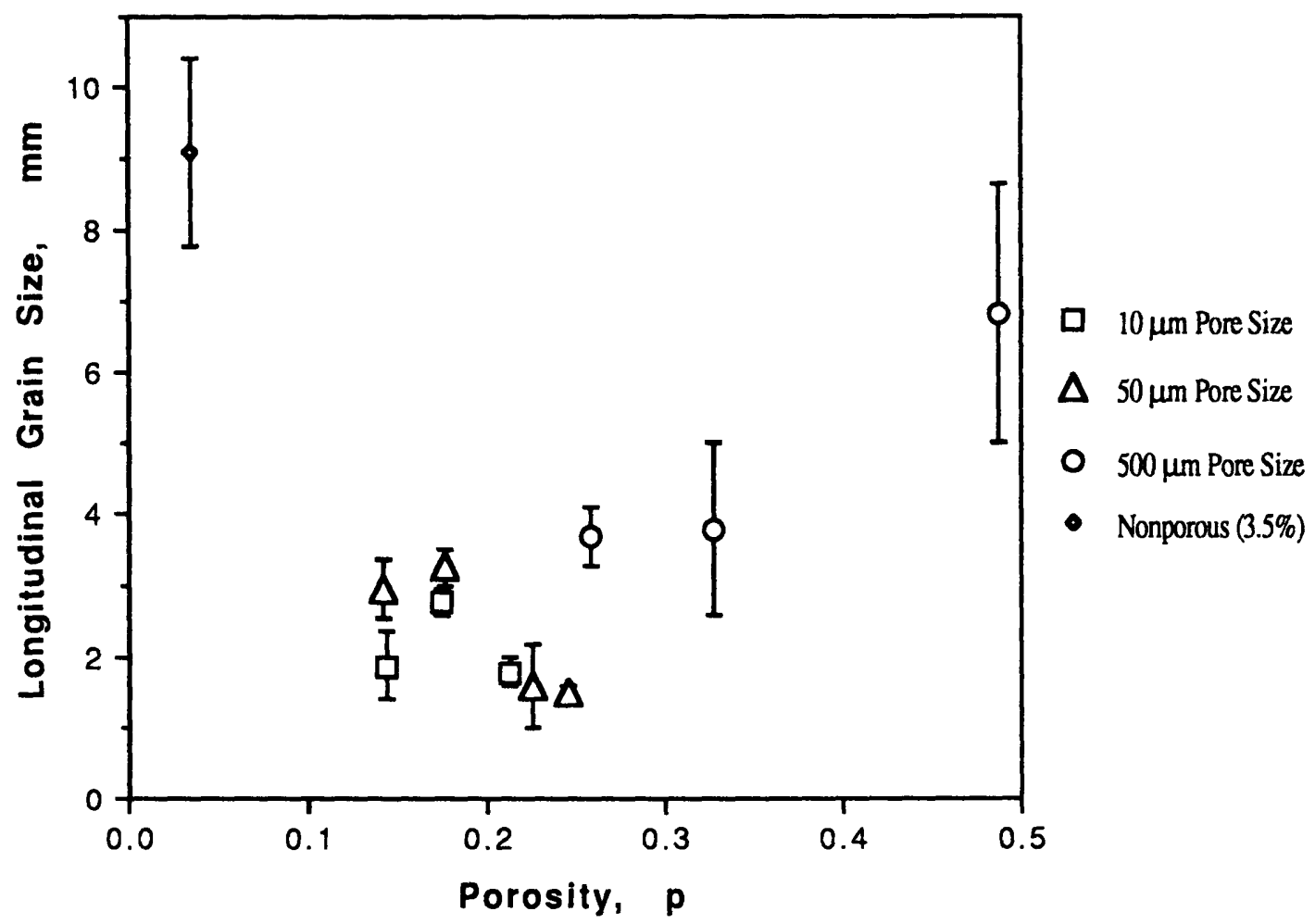

Figure 3.22 - Longitudinal Grain Size vs. Porosity

Data was measured using the line-intercept method. Each data point represents a single specimen. The error bars indicate the standard deviation of the measurements taken from each specimen. 
grain size. The result of this fact is that the aspect ratio (length / diameter) of the columnar grains is fairly consistent, averaging approximately 10 .

\subsection{Microstructural Characterization}

The following section presents several issues in the characterization of the GASAR porous samples with respect to porosity and pore size.

\subsubsection{Relationship Between Pore Structure and Grain Structure}

In the case of both the $10 \mu \mathrm{m}$ and $50 \mu \mathrm{m}$ nominal pore size specimens, the grain size was determined to be much larger than the pore size. Pores generally exist on the grain boundaries, or are contained within grains. The ring-like arrangements of pores observed at lower porosities ( see Figures $3.14 \mathrm{a}$ and $3.15 \mathrm{a}$ ) were originally thought to directly correspond to grain boundaries, but it was found that a typical grain would contain several of these rings. At higher porosities the pores tend to be more evenly dispersed. The grain boundary generally tends to traverse the shortest distance between pores. This results in an angular and irregular shape in both the transverse and longitudinal cross-sections. At lower porosities, the grain boundaries usually intersected the boundaries of the pore rings, while at higher porosities, the grain boundaries formed a more random pattern through the distributed pores.

In the case of the $500 \mu \mathrm{m}$ nominal pore size specimens, the grains were generally only slightly larger than the pores. In some cases, a grain contained only a single large pore, while in others, a number of smaller pores were contained. Grain boundaries generally traversed the shortest distance between large pores.

In longitudinal views of all specimens, grain boundaries could be typically seen running down the length of oriented pores. This indicates that a pore which is first nucleated on the grain boundary will remain on the boundary for its whole length. 


\subsubsection{Characterization of the Grain Boundary}

Because a number of effects related to grain size deal with interaction between grains across the grain boundary, it was desired to characterize the boundary itself with respect to pore content. A free surface has a very different effect than a solid boundary on the movement of dislocations during plastic deformation. Because of this, the percentage of the grain boundary that consisted of solid interface could influence the yielding behavior of the material. This percentage was measured from numerous micrographs taken of each specimen. The total grain boundary length was measured assuming that the boundary passed through the center of each pore on the boundary. The ratio of the solid line length to this total boundary length was taken as the percentage of the boundary area that is solid interface. This assumes that the porosity is consistent along the length of the specimen, which was verified with sectional porosity measurements.

The percentage of solid interface on the grain boundary is plotted as a function of porosity in Figure 3.23. From this plot it can be seen that this value is independent of porosity below a porosity of $\approx 0.30$. This indicates that the distribution of pores on the grain boundary is consistent. With increasing porosity, the characteristics of the grain boundaries do not change. Only the amount of grain boundary (due to smaller grains) and the number of pores contained within grains increase. In contrast to the smaller pore size specimens, the $500 \mu \mathrm{m}$ nominal pore size specimens show a slight decrease in solid interface percentage with increasing porosity.

\subsubsection{The Measured Pore Size and Size Distribution}

An estimate of the actual average pore size and size distribution of pores was made for each specimen on the transverse plane. In general, the average actual pore size for the nominal $10 \mu \mathrm{m}$ pore specimens was between $20 \mu \mathrm{m}-25 \mu \mathrm{m}$, although pores 


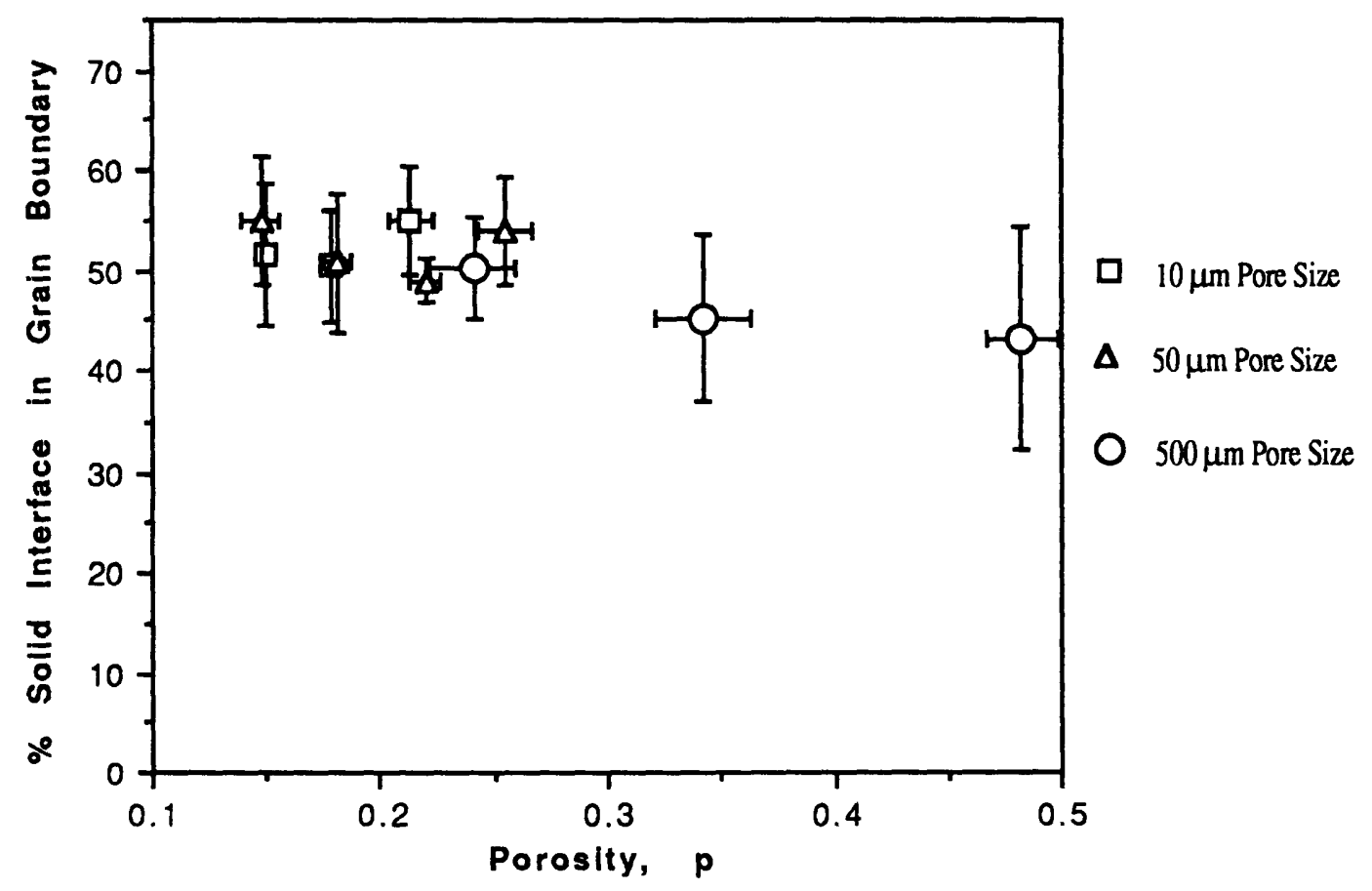

Figure 3.23 - Percentage of Grain Boundary Consisting of Solid Interface Between Adjacent Grains

Each data points indicates the statistical average of a large number of measurements made on 2-5 specimens. The error bars indicate the standard deviation with respect to each data point. 
from $10 \mu \mathrm{m}$ to $50 \mu \mathrm{m}$ were generally present. The nominal $50 \mu \mathrm{m}$ pore size specimens had an average pore size between $30 \mu \mathrm{m}$ and $35 \mu \mathrm{m}$, with the overall pore size range being from $10 \mu \mathrm{m}$ to $60 \mu \mathrm{m}$. In several $10 \mu \mathrm{m}$ and $50 \mu \mathrm{m}$ pore size specimens, a small number of pores between $150 \mu \mathrm{m}$ and $200 \mu \mathrm{m}$ in size could be found. No direct correlation between the existence of these pores, referred to a macropores, and the mechanical properties of the specimen was found.

The average actual pore size of the nominal $500 \mu \mathrm{m}$ pore size specimens was consistently between $150 \mu \mathrm{m}$ and $200 \mu \mathrm{m}$. The range of pore sizes present was between 50 and $500 \mu \mathrm{m}$. A summary of the actual pore size measurements as a function of porosity and nominal pore size can be found in Table 3.1.

$\begin{array}{cccc}\text { Porosity } & \begin{array}{c}\text { Nominal Pore } \\ \text { Size } \\ 10 \mu \mathrm{m}\end{array} & \begin{array}{c}\text { Measured Pore } \\ \text { Size } \\ 25 \mu \mathrm{m}\end{array} & \text { Pore Size Range } \\ 0.149 & 10 \mu \mathrm{m} & 25 \mu \mathrm{m} & 10 \mu \mathrm{m}-50 \mu \mathrm{m} \\ 0.179 & 10 \mu \mathrm{m} & 25 \mu \mathrm{m} & 10 \mu \mathrm{m}-50 \mu \mathrm{m} \\ 0.213 & 50 \mu \mathrm{m} & 30 \mu \mathrm{m} & 15 \mu \mathrm{m}-50 \mu \mathrm{m} \\ 0.148 & 50 \mu \mathrm{m} & 35 \mu \mathrm{m} & 15 \mu \mathrm{m}-60 \mu \mathrm{m} \\ 0.182 & 50 \mu \mathrm{m} & 35 \mu \mathrm{m} & 20 \mu \mathrm{m}-60 \mu \mathrm{m} \\ 0.220 & 50 \mu \mathrm{m} & 35 \mu \mathrm{m} & 20 \mu \mathrm{m}-60 \mu \mathrm{m} \\ 0.255 & 500 \mu \mathrm{m} & 150 \mu \mathrm{m} & 150 \mu \mathrm{m}-500 \mu \mathrm{m} \\ 0.241 & 500 \mu \mathrm{m} & 175 \mu \mathrm{m} & 150 \mu \mathrm{m}-500 \mu \mathrm{m} \\ 0.342 & 500 \mu \mathrm{m} & 200 \mu \mathrm{m} & 175 \mu \mathrm{m}-500 \mu \mathrm{m} \\ 0.482 & & & \end{array}$

Table 3.1 - Measured Transverse Pore Size

Each porosity value represents $2-5$ specimens of similar porosity. Measured values were taken from micrographs of the transverse cross section of each specimen. 


\subsubsection{Characterization Summary}

This section presents a series of diagrams summarizing the microstructural characterization data presented in the previous section. These diagrams are not meant to be an exact representation of any one specimen and are intended to act as a summary of the compiled measurements and observations for the purpose of discussion. The information accompanying each diagram are average values of a number of specimens with similar porosities. These characterization summary sketches are contained in Figures $3.24,3.25$, and 3.26. All of the sketches shown are drawn to the same scale (approximately $1 \mathrm{~cm}=150 \mu \mathrm{m}$ ).

\subsection{Hall-Petch Data}

In chapter 1, the Hall-Petch effect was discussed as a potential explanation of the mechanical behavior of the GASAR materials. In order to investigate the influence of the Hall-Petch effect, the yield strength of the GASAR tension specimens was plotted as a function of the inverse root of grain size. The transverse grain size was used in these plots because the transverse grain size more closely represents the path length traveled by dislocations during yielding, which is the primary factor in the Hall-Petch effect. The resulting plot is shown in Figure 3.27. A linear increase of yield strength with the inverse root grain diameter would indicate a strong influence of the Hall-Petch effect. A distinct increase of this sort can be seen between the nonporous specimens and the porous specimens, but no recognizable relationship can be seen in the porous specimens themselves. Figure 3.28 shows a comparison of the GASAR Hall-Petch data with HallPetch data for copper from other sources. Both of these sources are based on worked and annealed material with roughly equiaxed grains. The Carrecker and Hibbard ${ }^{29}$ yield strength values were measured using an offset strain of $0.5 \%$, while the Feltham and Meakin ${ }^{28}$ yield strength was taken as the intersection of tangent lines from the elastic and plastic regions of the stress-strain curve. In a material which strain hardens as 


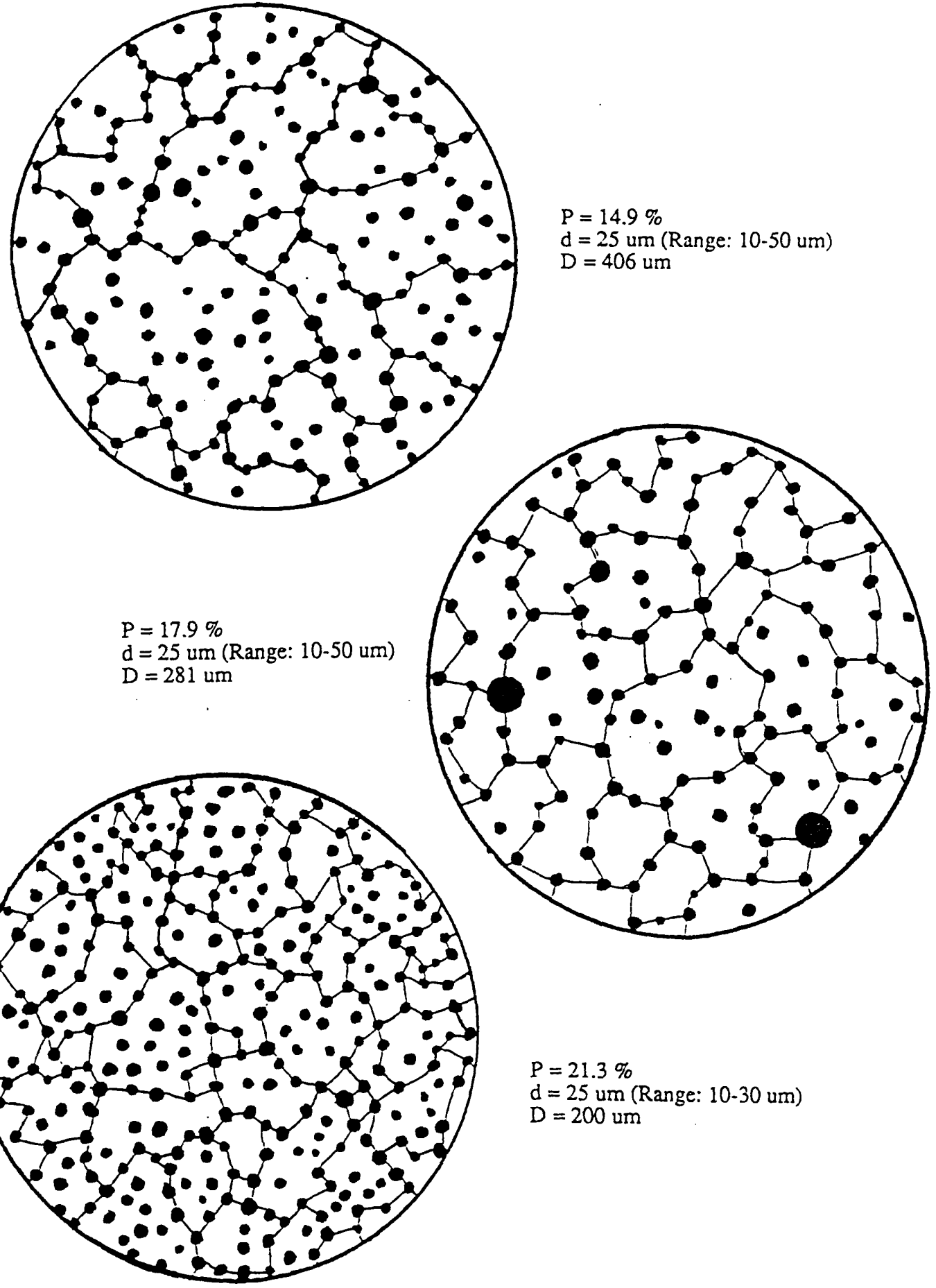

Figure 3.24 - Microstructural Characterization Summary for Specimens with a Nominal Pore Size of $10 \mu \mathrm{m}$

All characterization sketches are for the purpose of cualitative comparison only 

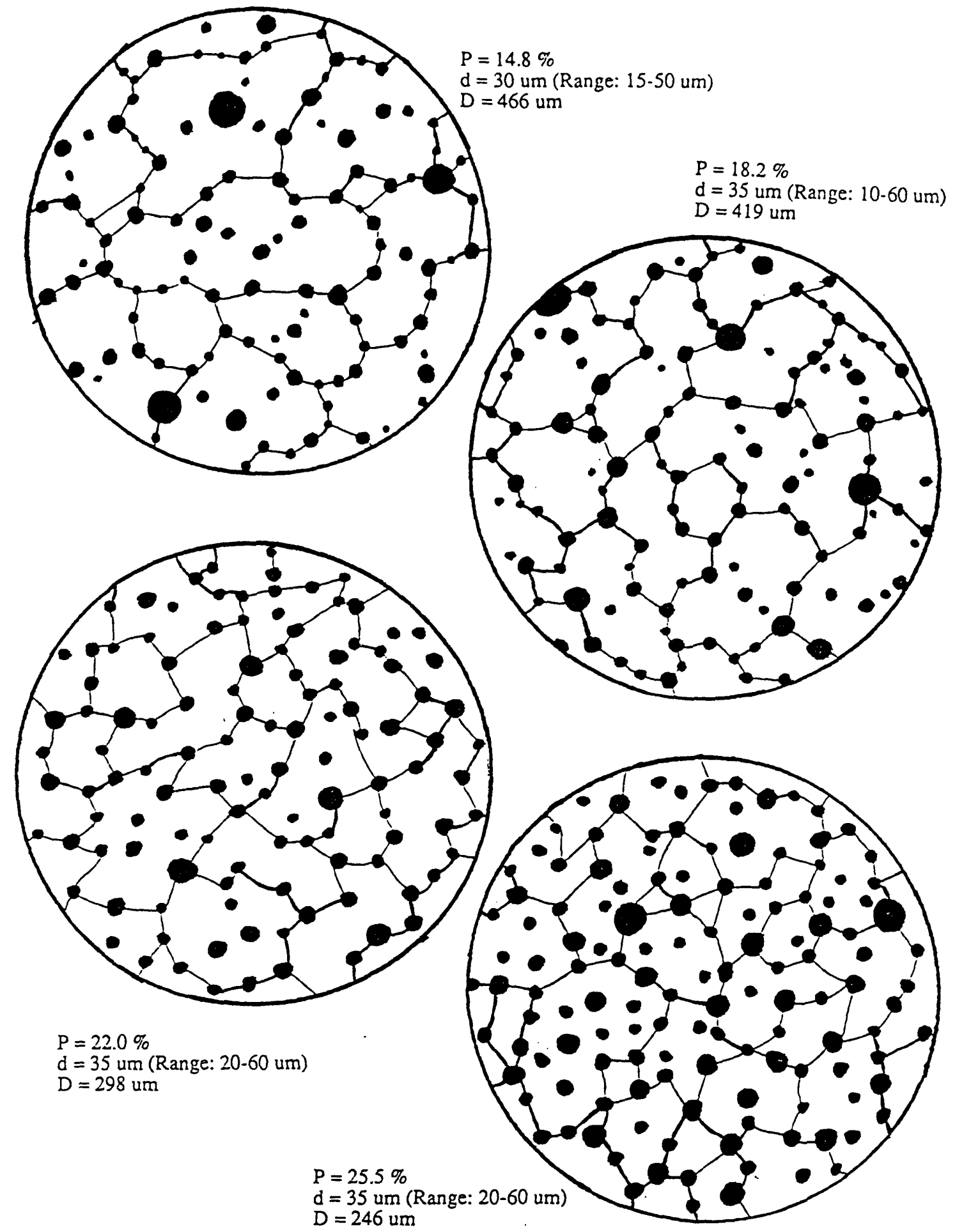

Figure 3.25 - Microstructural Characterization Summary for

Specimens with a Nominal Pore Size of $\mathbf{5 0 \mu \mathrm { m }}$

All characterization sketches are for the purpose of qualitative comparison only 


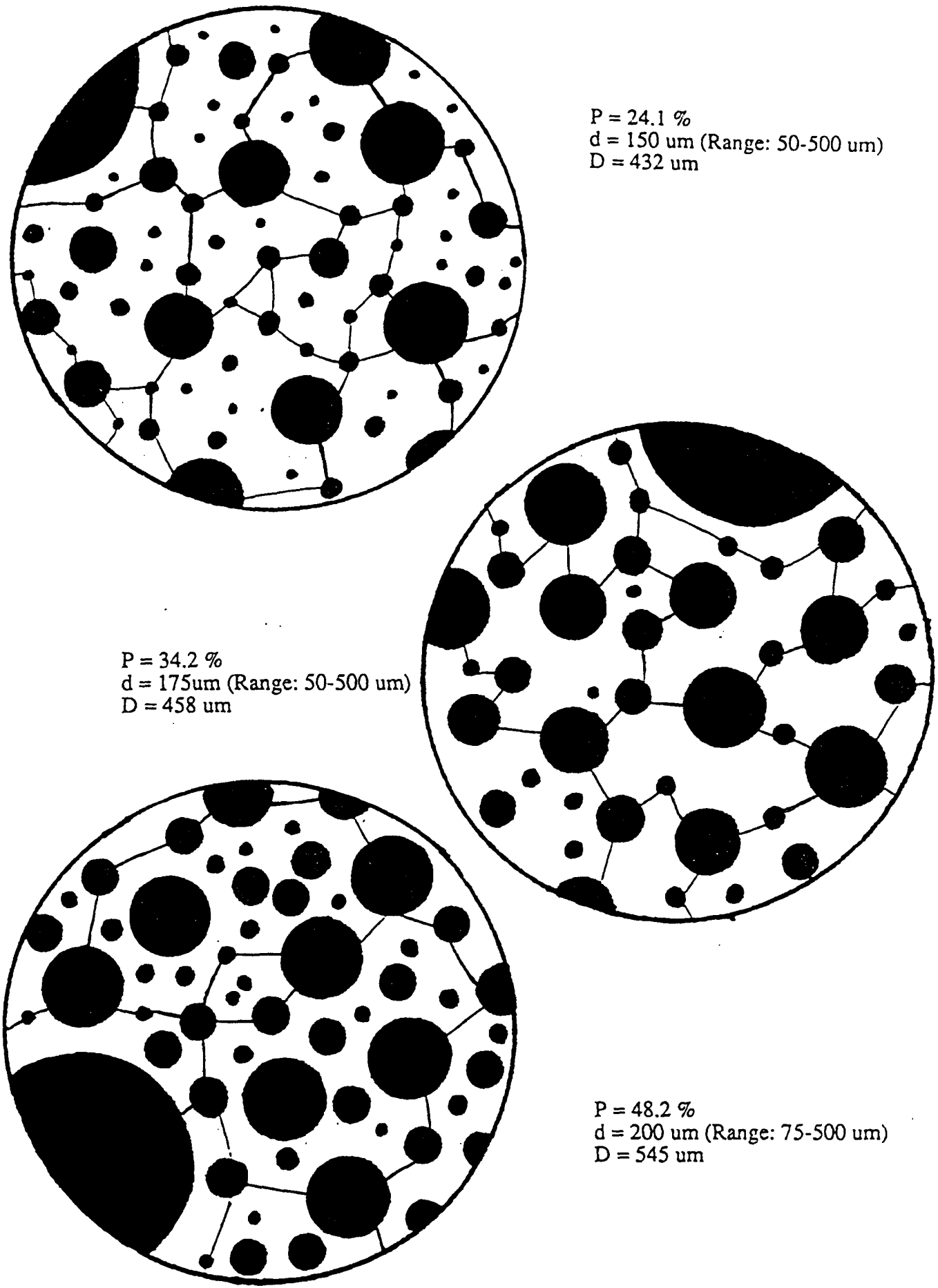

Figure 3.26 - Microstructural Characterization Summary for Specimens with a Nominal Pore Size of $500 \mu \mathrm{m}$

All characterization sketches are for the purpose of qualitative comparison only 


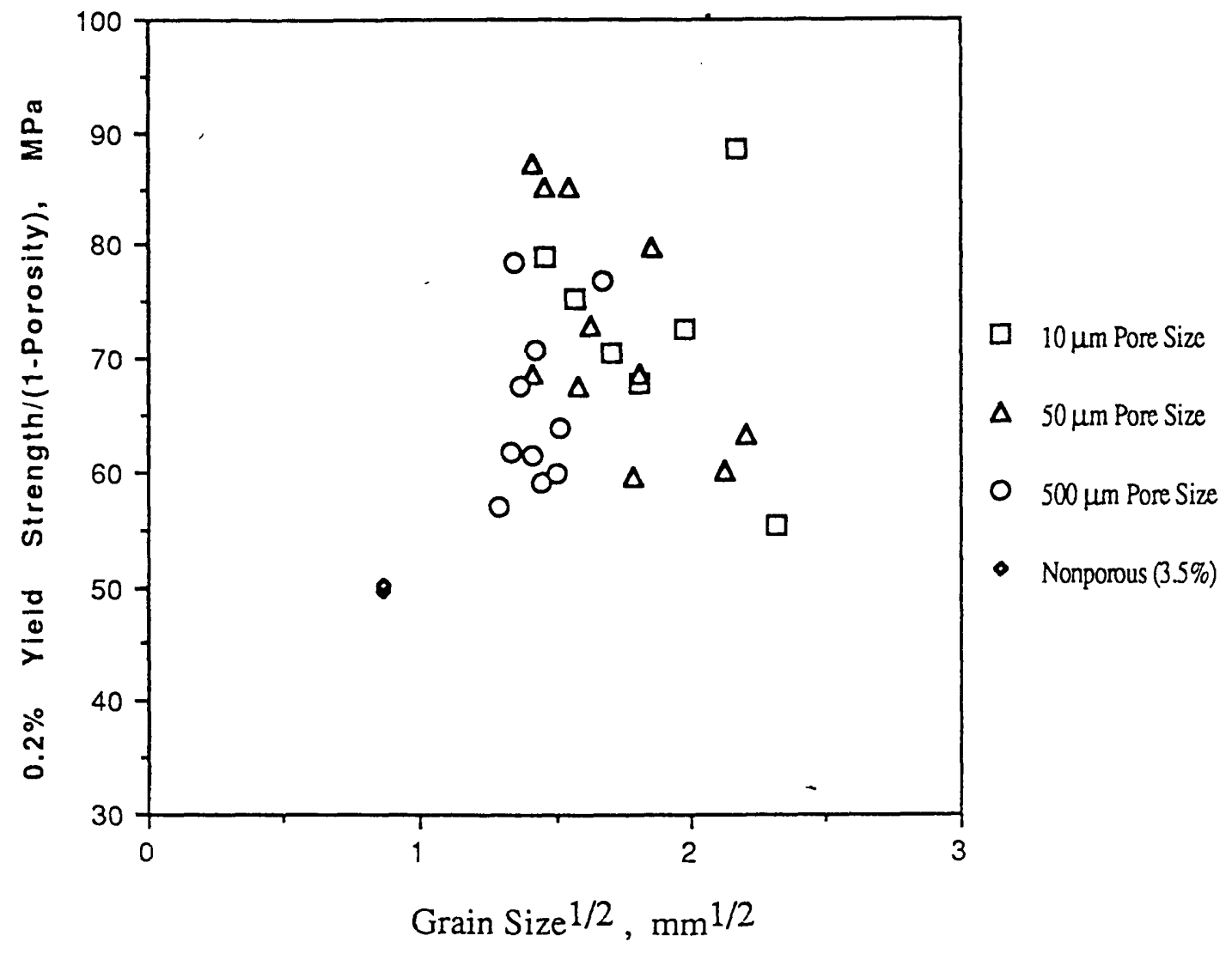

Figure 3.27 - Hall-Petch Data for GASAR Specimens

The grain diameter used is the transverse grain size. A straight line formed by this data would indicate a perfect correlation with the Hall Petch equation. 


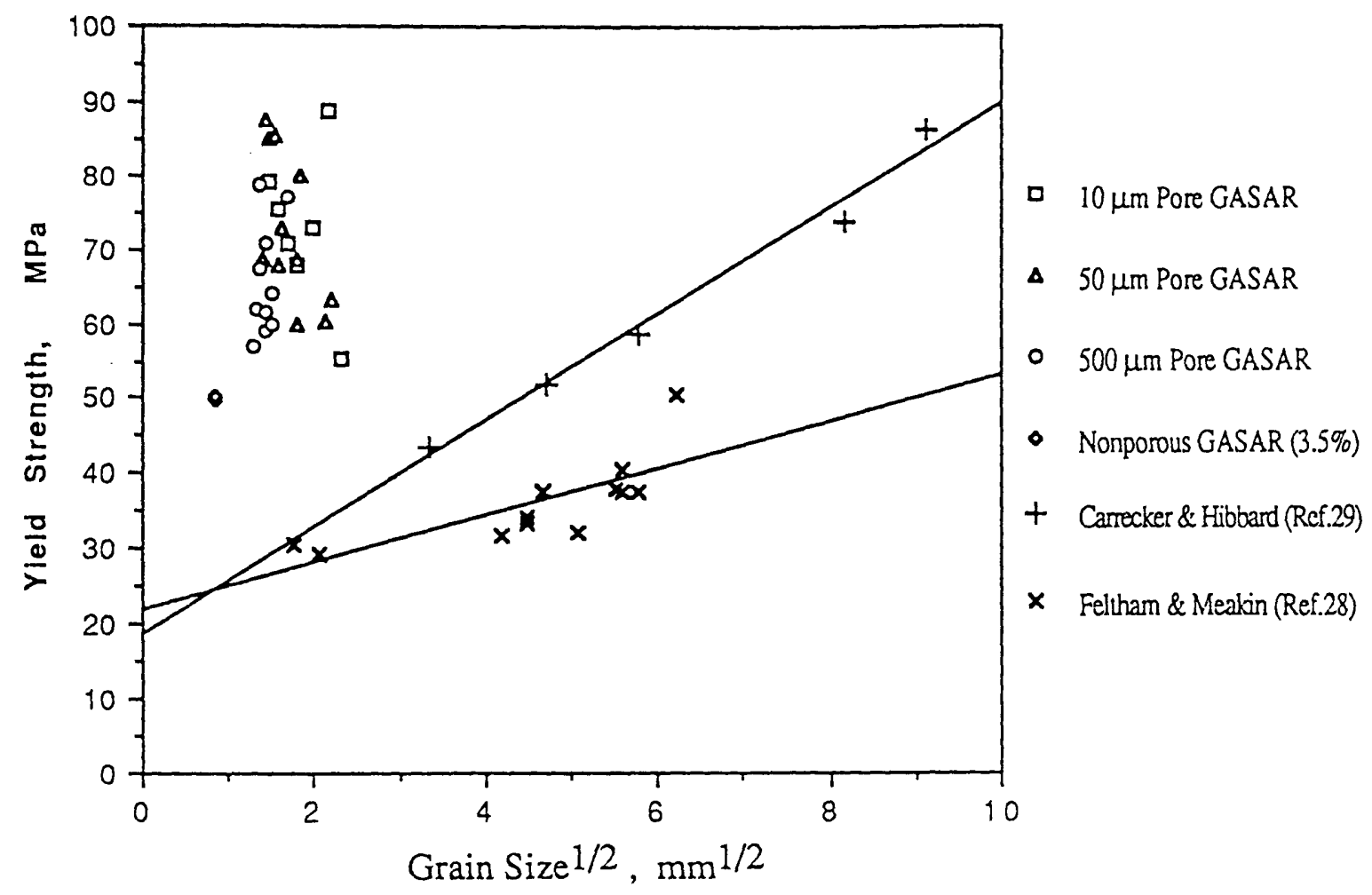

Figure 3.28 - Comparison of GASAR Data with Hall-Petch Data From Other Sources

All GASAR yield strength values were measured using a $0.2 \%$ offset strain. Carrecker and Hibbard data was measured using a $0.5 \%$ offset strain. Feltaham and Meakim data was measured by a linear interpolation method. 
appreciably as copper does, both of these methods of yield strength measurement would tend to report a larger value than would be found using a $0.2 \%$ offset. The yield strength values reported by both of the other sources, however, are significantly lower than those of the nonporous and porous GASAR specimens at similar grain diameters. Note that this disparity would be even more pronounced if the longitudinal grain size were used for the GASAR data points.

\subsection{Grain Substructure}

Through microscopic examination of the etch pit patterns, it was concluded that a substructure of low-angle grain boundaries, such as that discussed in section 1.6.1, exists within many of the columnar grains in the nonporous material. These low-angle boundaries form columnar sub-grains oriented in the longitudinal direction. This substructure was not found in all of the grains, however. The porous GASAR specimens also showed no distinct grain substructure. Micrographs of the etch pit patterns used to evaluate the grain substructure are shown in Figure 3.29. Picture (a) shows the pattern found in a nonporous specimen grain with a developed grain substructure. Picture (b) shows the pattern found in the porous materials. Note that the depressions in picture (b) are etch pits and not micropores.

\subsection{Results of Texture Analysis}

The results of the x-ray diffractometer tests show strong preferred orientation in both the nonporous and porous specimens. Sample diffractometer plots are shown in Figure 3.30. The relative peak size distribution that would be expected in the plot of a randomly oriented copper sample is also shown. Transverse cross-sectional samples were used in the diffractometer plots shown. In both the nonporous and porous materials, the $<100>$ crystallographic direction tends to be parallel to the longitudinal axis of the specimen. This $<100>$ texture in the solidification direction is consistent with past studies involving FCC materials in unidirectional solidification ${ }^{7}$. It is important to 
Figure 3.29 - Grain Substructure Micrographs

a) Nonporous Specimen, b) $10 \mu \mathrm{m}$ Nominal Pore Size, Porosity $=0.154$

a)

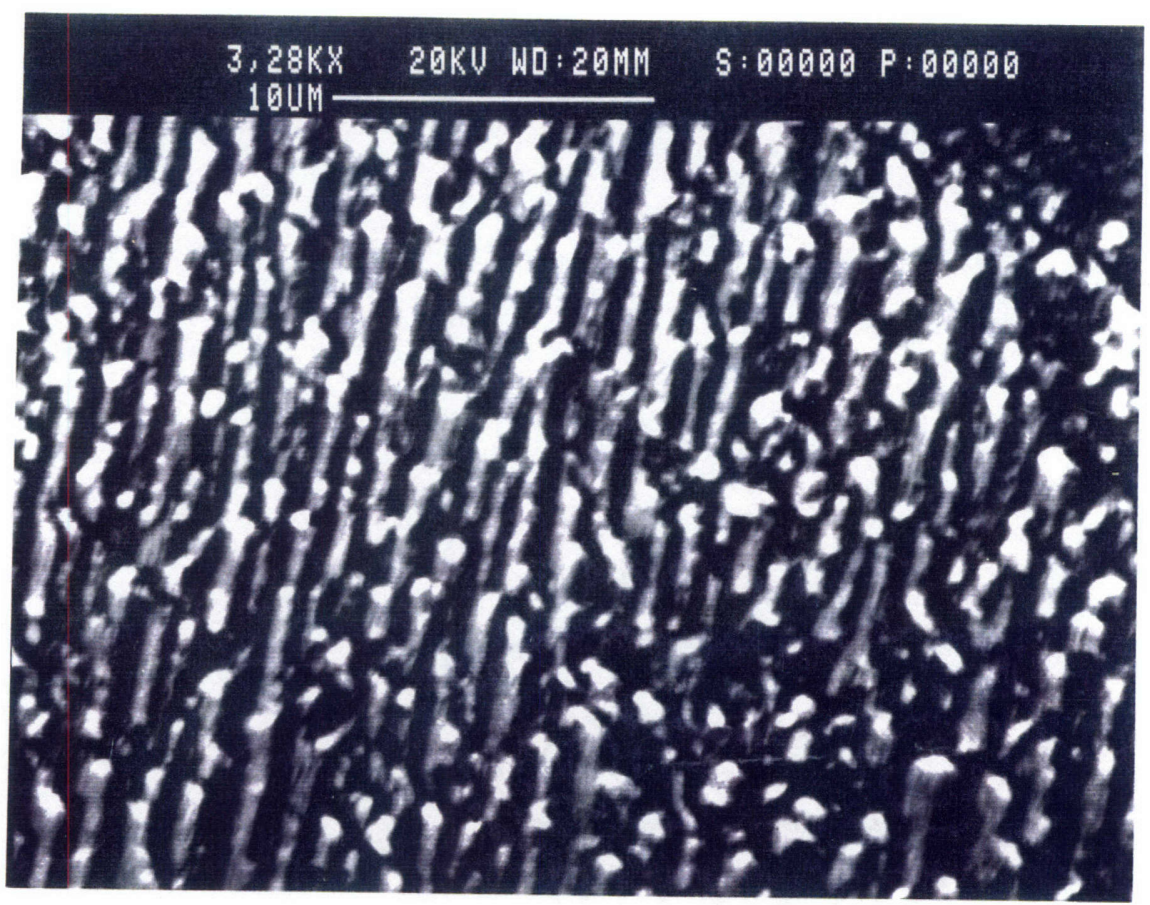

b)

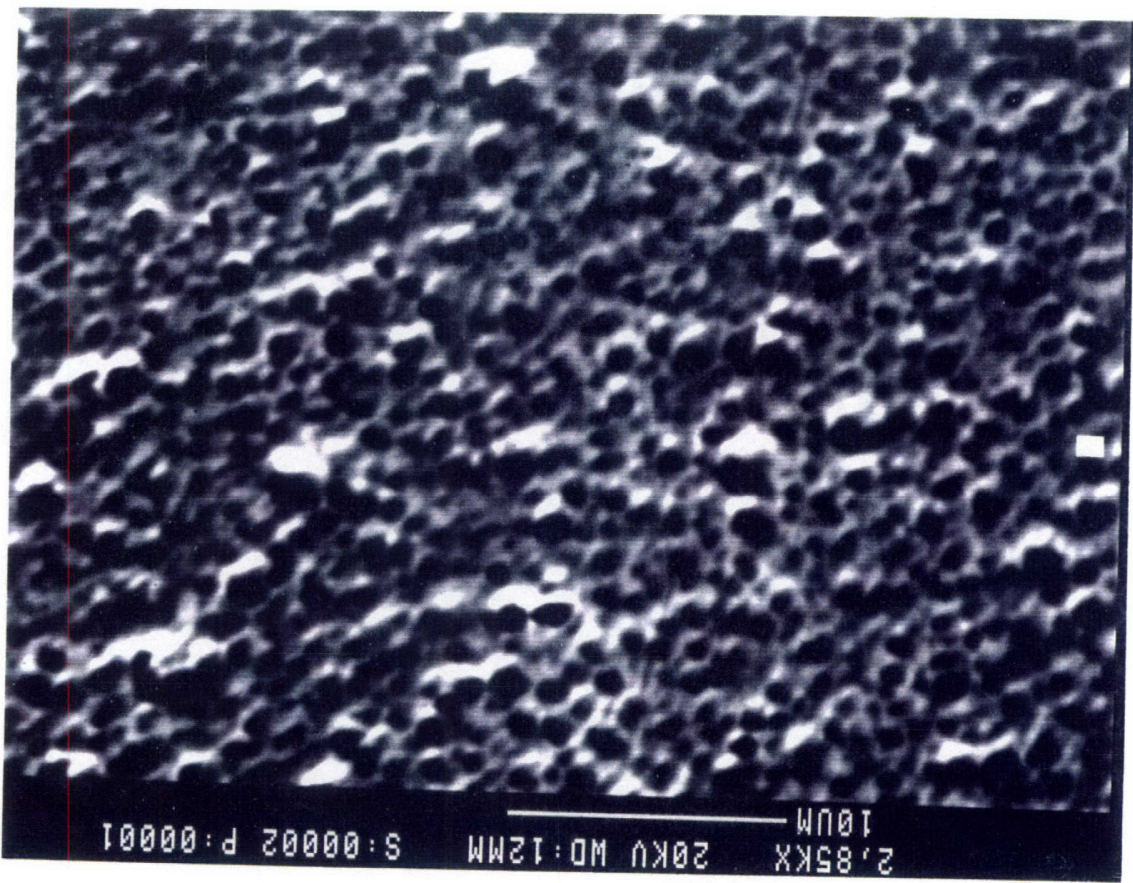




\section{Figure 3.30 - Sample Diffactometer Test Results}

a) Peak distribution for copper with random crystallographic orientation,

b) Nonporous specimen, c) $10 \mu \mathrm{m}$ nominal pore size specimen, d) $50 \mu \mathrm{m}$ nominal pore size specimen, e) $500 \mu \mathrm{m}$ nominal pore size specimen.

a)

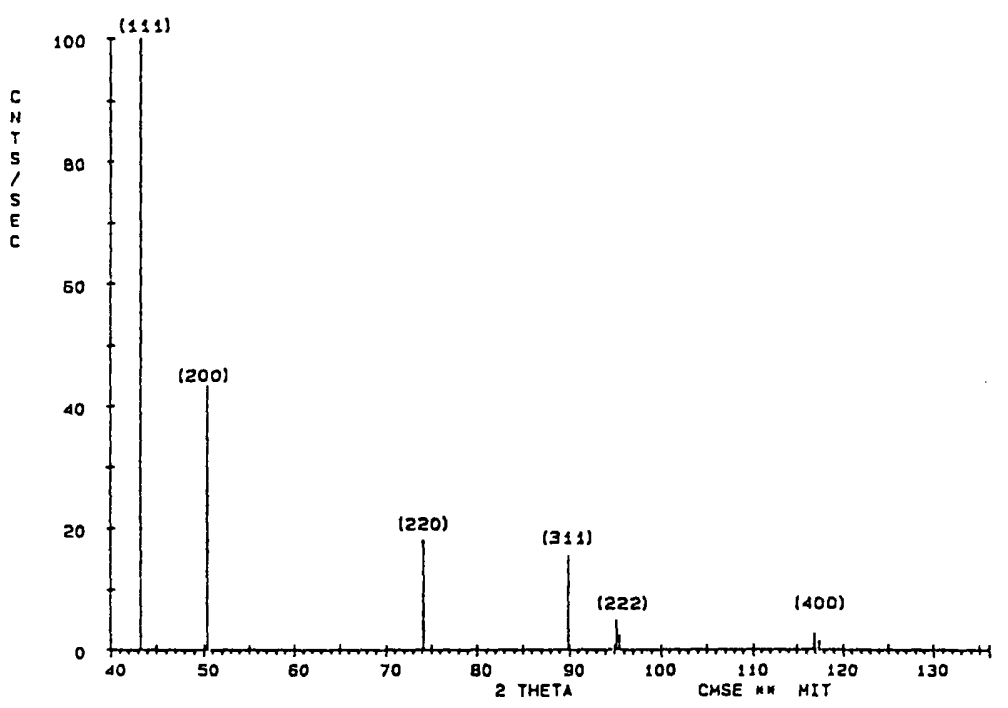

b)

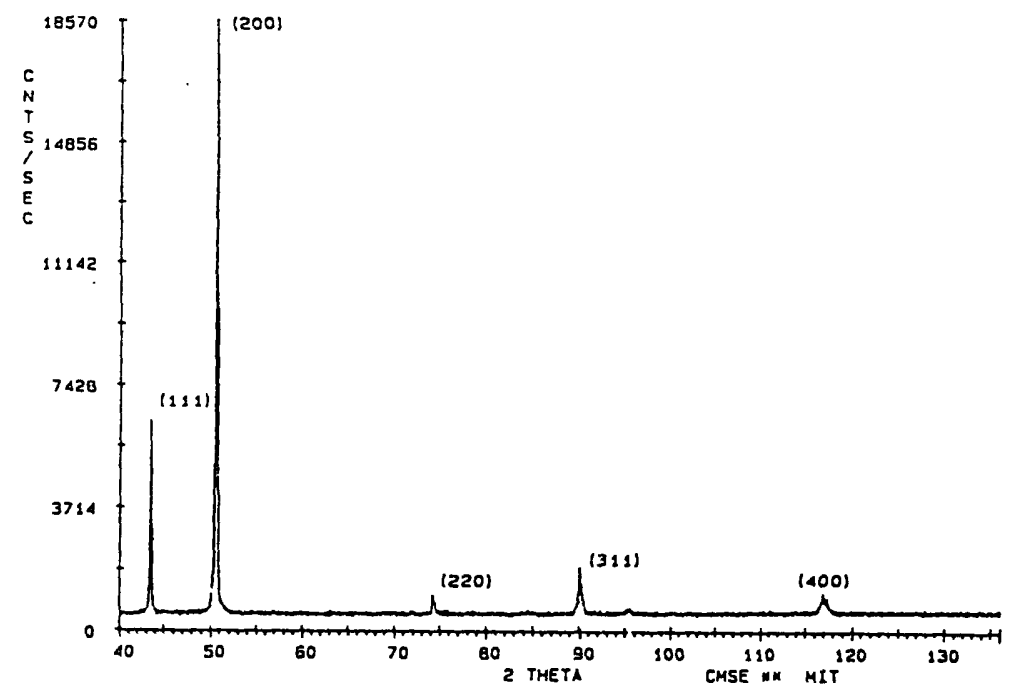


Figure 3.30 - Sample Diffactometer Test Results (continued)

c)

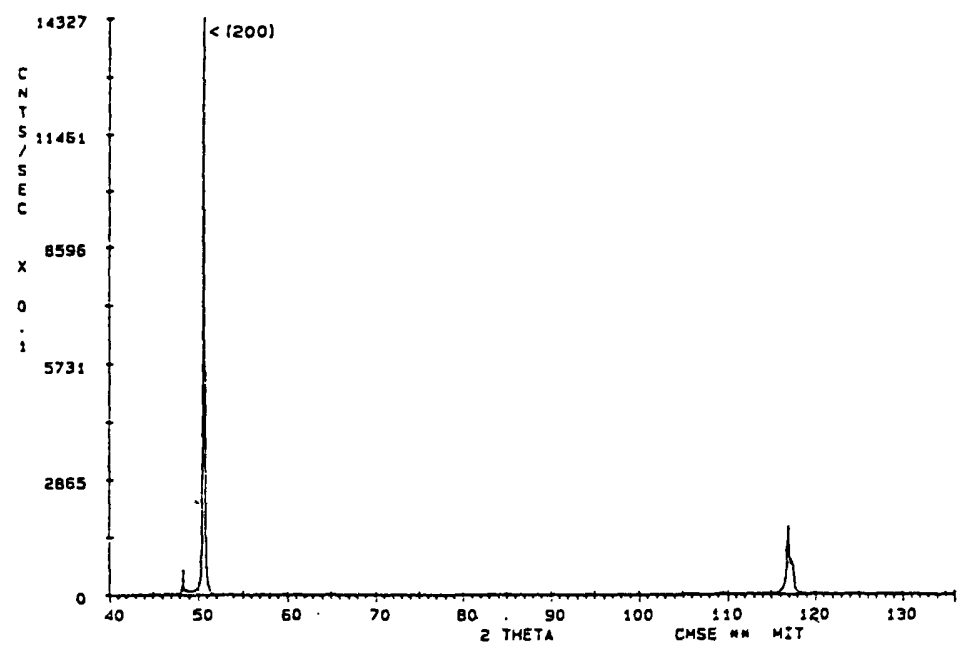

d)
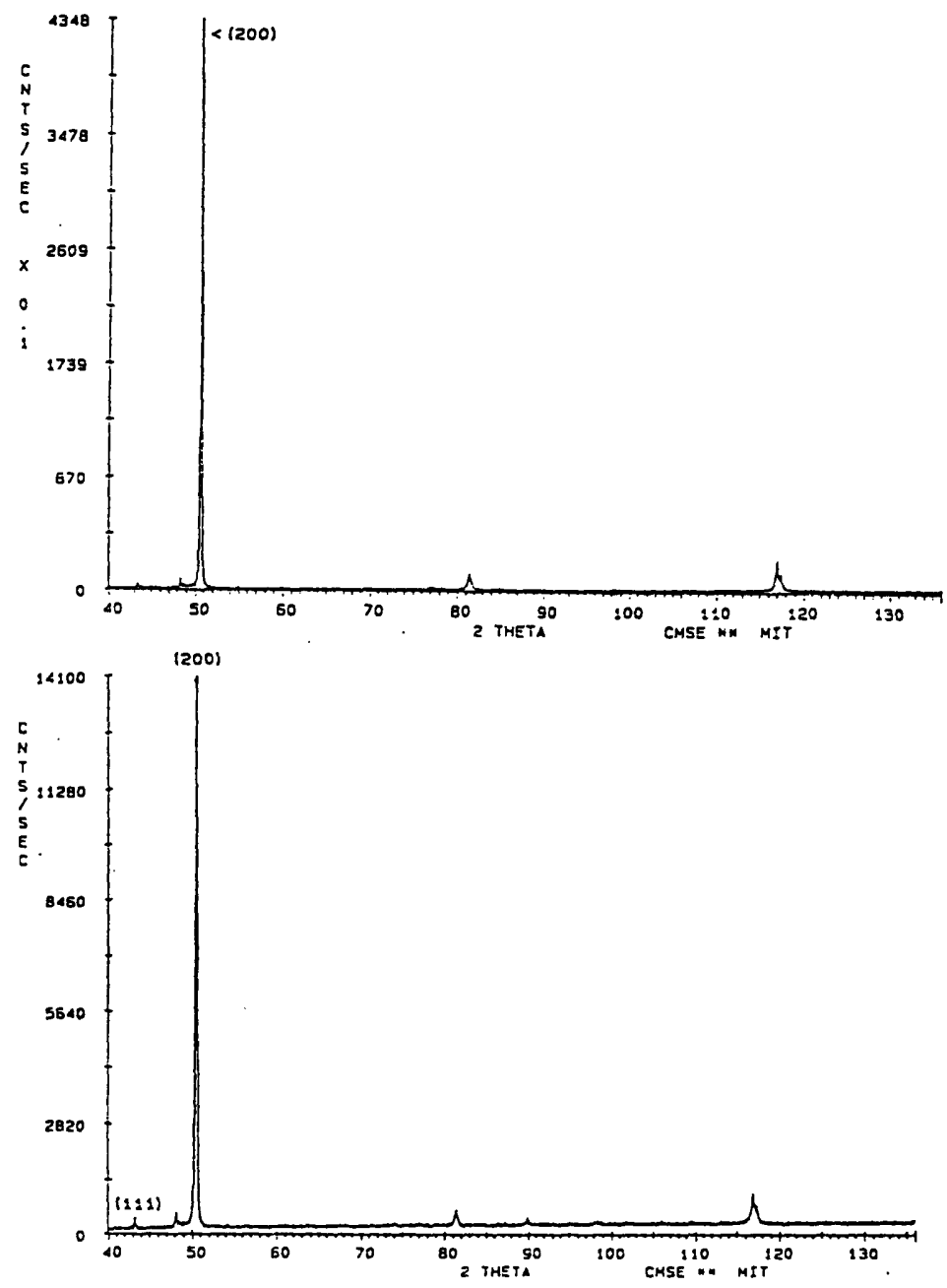
note that although a strong preferred orientation is shown, that grains of other orientations are present in all specimens. Diffractometer tests do not give a direct quantitative analysis of the relative number of grains of various orientations present in a particular cross-section, but it is obvious from the relative peak size that the number of non- $<100>$ grains present varies from specimen to specimen. Although the texture is predominantly $\langle 100\rangle$, the large size of individual grains with respect to the testing specimen size increase the possible effect of the orientation of a single grain on the measured properties. The peak size distributions of the graphs in Figure 3.30 indicate that the $<100\rangle$ texture is somewhat more pronounced in the porous specimens than in the nonporous material.

\subsection{Results of Impurity Testing}

The results of the electron probe tests showed that both the porous and nonporous GASAR materials are composed of copper at a purity of higher than $99 \%$. A sample electron probe plot is shown in Figure 3.31. Small particle impurities of various elements were found sporadically, although these particles were not frequent enough to cause any significant strengthening effect. Traces of alumina were found on the interior walls of some pores.

A detailed composition breakdown was obtained by ONR researchers for both porous and nonporous specimens of the same material used in the experimental program of this study ${ }^{41}$. The results indicate a purity of approximately $99.98 \%$ for both the nonporous and porous materials. The porous specimen was found to have a higher impurity concentration at the end sections than at the center. With respect to the impurity elements present, only slight differences were found between the porous and nonporous specimens. The results of the impurity testing are summarized in Table 3.2. 
Figure 3.31 - Sample Electron Probe Results

Purity of greater than $99 \%$ is indicated

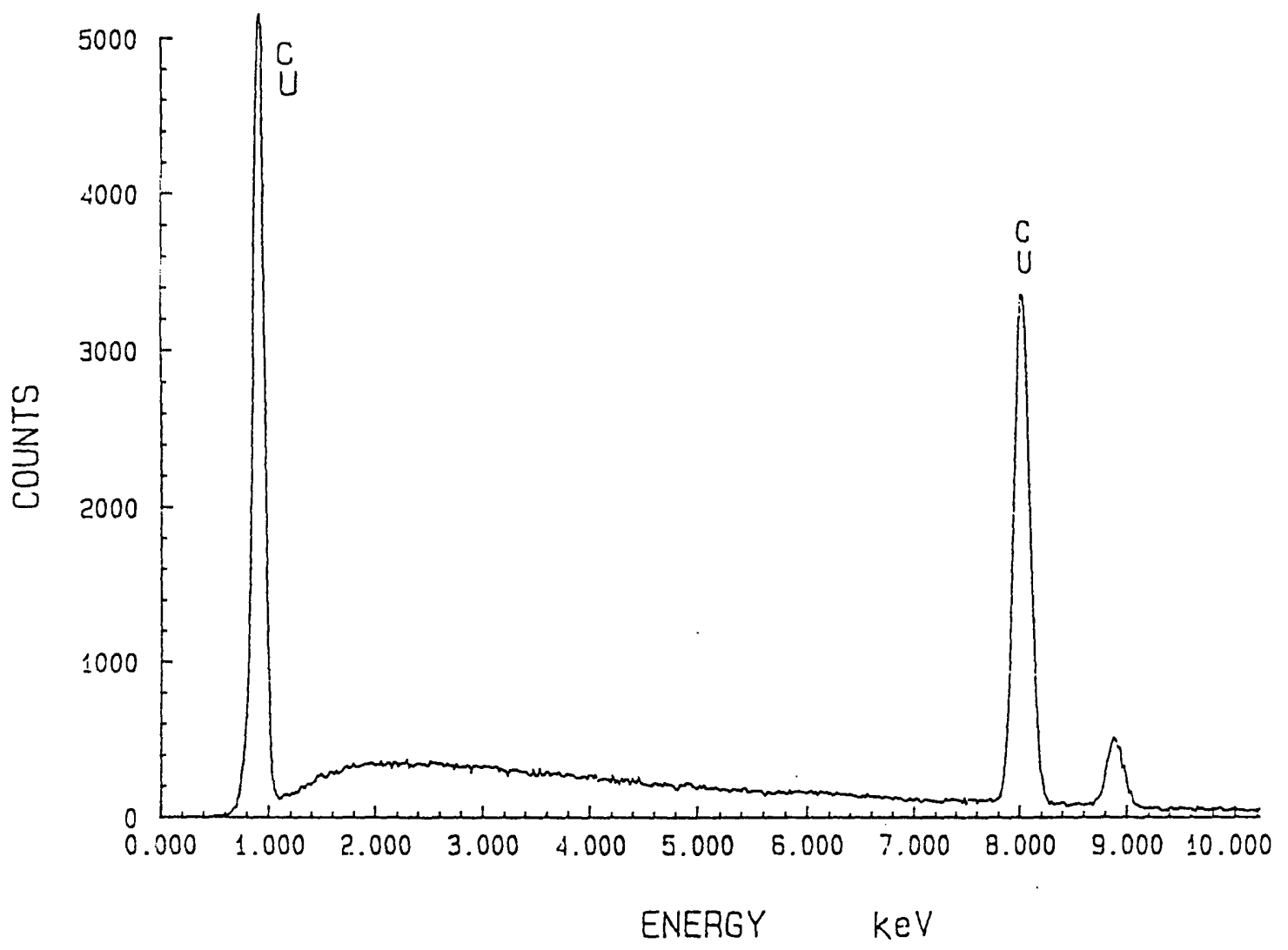




\begin{tabular}{|c|c|c|c|c|}
\hline & \multicolumn{4}{|c|}{ All Impurity Content Values in Weight Percentage } \\
\hline Impurity & $30 \%$ norous & $30 \%$ norous & $30 \%$ norous & Nonnorous \\
\hline Element & Oum Pore Size & Oum Pore Size & Oum Pore Size & Specimen \\
\hline & Top Section & Middle Section & Bottom Section & \\
\hline Aluminum & $<0.0005$ & $<0.0005$ & $<0.0005$ & $<0.0005$ \\
\hline Carbon & $<0.002$ & $<0.002$ & $<0.002$ & 0.001 \\
\hline Chromium & 0.0003 & 0.0006 & 0.0003 & $<.0003$ \\
\hline Iron & 0.0056 & 0.0055 & 0.0051 & 0.0094 \\
\hline Phosphorus & $<0.001$ & $<0.001$ & $<0.001$ & $<0.001$ \\
\hline Silicon & $<0.0005$ & $<0.0005$ & $<0.0005$ & $<0.0005$ \\
\hline Silver & 0.0005 & 0.0005 & 0.0004 & $<0.0003$ \\
\hline Sulfur & 0.0005 & 0.0005 & $<0.0005$ & 0.0012 \\
\hline Tellurium & $<0.001$ & $<0.001$ & $<0.001$ & $<0.001$ \\
\hline Zirconium & $<0.0005$ & $<0.0005$ & $<0.0005$ & $<0.0005$ \\
\hline Hydrogen & 0.0022 & 0.0025 & 0.0022 & 0.0003 \\
\hline Nitrogen & 0.0005 & 0.0007 & 0.0006 & 0.0003 \\
\hline Oxygen & 0.0025 & 0.0028 & 0.0032 & 0.0032 \\
\hline Alumina & 0.0056 & $<0.001$ & 0.0055 & 0.0023 \\
\hline Calcium & 0.0014 & 0.0013 & 0.0014 & 0.0013 \\
\hline Lead & 0.0015 & 0.0014 & 0.0014 & 0.0012 \\
\hline Total Impurity & $<0.027$ & $<0.0123$ & $<0.0261$ & $<0.024$ \\
\hline Percentage & & & & \\
\hline
\end{tabular}

Table 3.2 - Results of Impurity Analysis

Testing was done on a nonporous copper specimen and on the top, bottom, and center sections of a GASAR porous copper specimen. (Ref. 41) 


\section{Appendix to Chapter Three :}

\section{Testing Data}

Table 3.A.1 - Tensile Test Data

\begin{tabular}{|c|c|c|c|c|c|c|c|c|}
\hline $\begin{array}{c}\text { Porosity } \\
p\end{array}$ & $\begin{array}{l}\text { Nominal } \\
\text { Pore Size }\end{array}$ & $\begin{array}{c}0.2 \% \text { Yield } \\
\text { Strength } \\
\mathrm{MPa}\end{array}$ & $\begin{array}{l}\text { Ult.Tensile } \\
\text { Strength } \\
\text { MPa }\end{array}$ & $\begin{array}{c}\text { Young's } \\
\text { Modulus } \\
\text { GPa }\end{array}$ & $\begin{array}{c}0.2 \% Y S /(1-p) \\
M P a\end{array}$ & UTS/(1-p) & $\begin{array}{c}E /(1-p) \\
M P a\end{array}$ & $\begin{array}{c}\text { Strain at } \\
\text { Peak Stres }\end{array}$ \\
\hline 0.151 & 10 & 64.0 & 133.0 & 32.5 & 75.4 & 156.6 & 38.3 & 0.151 \\
\hline 0.154 & 10 & 67.0 & 141.3 & 58.0 & 79.2 & 166.9 & 68.5 & 0.146 \\
\hline 0.144 & 10 & 60.5 & 133.6 & 89.6 & 70.7 & 156.1 & 104.7 & 0.198 \\
\hline 0.175 & 10 & 60.0 & 129.3 & 32.9 & 72.7 & 156.7 & 39.9 & 0.148 \\
\hline 0.210 & 10 & 70.0 & 123.3 & 56.8 & 88.7 & 156.2 & 72.0 & 0.123 \\
\hline 0.205 & 10 & 59.0 & 123.3 & 60.6 & 74.2 & 155.0 & 76.2 & 0.147 \\
\hline 0.224 & 10 & 43.0 & 114.2 & 58.0 & 55.4 & 147.1 & 74.7 & 0.148 \\
\hline 0.182 & 10 & 55.5 & 112.0 & 59.1 & 67.9 & 137.0 & 72.3 & 0.082 \\
\hline$Q .179$ & 50 & 70.0 & 139.6 & 88.6 & 85.3 & 170.0 & 107.9 & 0.132 \\
\hline 0.144 & 50 & 58.0 & 137.8 & 92.4 & 67.7 & 160.9 & 107.9 & 0.180 \\
\hline 0.142 & 50 & 75.0 & 131.0 & 50.0 & 87.4 & 152.6 & 58.2 & 0.122 \\
\hline 0.157 & 50 & 58.0 & 125.2 & 57.8 & 68.8 & 148.6 & 68.6 & 0.188 \\
\hline 0.189 & 50 & 69.0 & 127.3 & 91.5 & 85.0 & 156.9 & 112.7 & 0.155 \\
\hline 0.178 & 50 & 60.0 & 127.9 & 60.1 & 73.0 & 155.6 & 73.1 & 0.206 \\
\hline 0.225 & 50 & 62.0 & 118.2 & 40.4 & 80.0 & 152.5 & 52.1 & 0.110 \\
\hline 0.215 & 50 & 54.0 & 122.1 & 45.5 & 68.8 & 155.5 & 58.0 & 0.177 \\
\hline 0.246 & 50 & 45.0 & 104.9 & 61.8 & 59.7 & 130.1 & 82.0 & 0.125 \\
\hline 0.268 & 50 & 44.0 & 101.5 & 81.8 & 60.1 & 138.7 & 111.8 & 0.114 \\
\hline 0.250 & 50 & 47.5 & 104.8 & 45.2 & 63.3 & 139.7 & 60.2 & 0.092 \\
\hline 0.244 & 500 & 46.5 & 97.3 & 62.7 & 61.5 & 128.7 & 82.9 & 0.113 \\
\hline 0.257 & 500 & 44.5 & 91.6 & 41.1 & 59.9 & 123.4 & 55.4 & 0.144 \\
\hline 0.221 & 500 & 60.0 & 111.7 & 39.4 & 77.0 & 143.3 & 50.5 & 0.103 \\
\hline 0.327 & 500 & 43.0 & 88.9 & 75.8 & 63.9 & 132.1 & 112.6 & 0.064 \\
\hline 0.357 & 500 & 38.0 & 74.8 & 37.5 & 59.1 & 116.2 & 58.3 & 0.073 \\
\hline 0.487 & 500 & 40.3 & 51.0 & & 78.5 & 99.3 & & 0.017 \\
\hline 0.482 & 500 & 35.0 & 47.0 & 31.8 & 67.5 & 90.7 & 61.4 & 0.027 \\
\hline 0.460 & 500 & 38.2 & 54.0 & 65.4 & 70.8 & 100.1 & 121.2 & 0.026 \\
\hline 0.504 & 500 & 28.3 & 39.4 & & 57.1 & 79.5 & & 0.036 \\
\hline 0.479 & 500 & 32.2 & 47.4 & 28.1 & 61.7 & 90.9 & 53.9 & 0.036 \\
\hline 0.035 & Nonporous & 48.0 & 139.0 & 66 & 49.7 & 144.0 & 68.4 & 0.316 \\
\hline 0.035 & Nonporous & 48.5 & 136.7 & 67 & 50.3 & 141.7 & 69.4 & 0.312 \\
\hline
\end{tabular}




\section{Table 3.A.2 - Microscopy Data}

$\begin{array}{ccccccc}\begin{array}{c}\text { Porosity } \\ p\end{array} & \begin{array}{c}\text { \# Spec. } \\ \text { N }\end{array} & \begin{array}{c}\text { Nominal } \\ \text { Pore Size } \\ (\mu \mathrm{m})\end{array} & \begin{array}{c}\text { Measured } \\ \text { Pore Size } \\ (\mu \mathrm{m})\end{array} & \begin{array}{c}\text { Pore Size } \\ \text { Range } \\ (\mu \mathrm{m})\end{array} & \begin{array}{c}\text { Transverse } \\ \text { Grain Size } \\ (u \mathrm{~m})\end{array} & \begin{array}{c}\text { Axial } \\ \text { Grain Size } \\ (\mu \mathrm{m})\end{array} \\ 0.150 & 3 & 10 & 25 & 10-50 & 406 & 1879 \\ 0.179 & 2 & 10 & 25 & 10-50 & 281 & 2781 \\ 0.213 & 3 & 10 & 25 & 10-30 & 200 & 1790 \\ 0.148 & 3 & 50 & 30 & 15-50 & 466 & 2964 \\ 0.182 & 3 & 50 & 35 & 15-60 & 419 & 3252 \\ 0.220 & 2 & 50 & 35 & 20-60 & 298 & 1609 \\ 0.255 & 3 & 50 & 35 & 20-60 & 246 & 1490 \\ 0.241 & 3 & 500 & 150 & 150-500 & 432 & 3676 \\ 0.342 & 2 & 500 & 175 & 150-500 & 458 & 3784 \\ 0.482 & 5 & 500 & 200 & 175-500 & 546 & 6819 \\ 0.035 & 2 & \text { Nonporous } & 750 & & 1350 & 9090\end{array}$




\section{Chapter Four: \\ Discussion of Results}

This chapter discusses the results presented in chapter three with respect to the issues introduced in chapter one.

\subsection{Discussion of Tensile Testing Results}

In chapter one, data recorded by DMI on the behavior of copper GASAR materials was presented. Referring back to Figures 1.5 and 1.6, the Ukrainian data indicated that the porous GASAR material could be up to $70 \%$ stronger, with respect to net area yield strength, and 15\% stronger, with respect to net area ultimate tensile strength, than nonporous copper. The questions raised by this data stemmed from the limited number of data points and the unknown origin of the values used for the reference yield and ultimate tensile strengths of solid copper. The reference values used were significantly lower than the standard reference strengths given for polycrystalline copper by a number of sources.

The strengthening effect reported by DMI was confirmed in tensile tests run on specimens with a reduced diameter of $5 \mathrm{~mm}$. GASAR porous specimens had a net area yield strength as much as $76 \%$ higher than the nonporous copper specimens. A similar increase of as much as $18 \%$ was found in the net area ultimate tensile strength. The measured strength values of the nonporous specimens tested are comparable to the nonporous copper strengths used by the DMI as reference values. This suggests that the nonporous material received from DMI is the same material used as a reference in Figures 1.5 and 1.6. The size of the specimens used by DMI is unknown, however. The size of 
the testing specimen may have a significant effect on the measured strength of GASAR specimens, particularly in the nonporous material (see section 4.2).

In general, the measured ultimate strength data is comparable to that of DMI. The Ukrainian values are slightly higher than the measured data, but for the $10 \mu \mathrm{m}$ and $50 \mu \mathrm{m}$ nominal pore size specimens, the measured data is usually within $10 \mathrm{MPa}$ of the DMI curve. The $500 \mu \mathrm{m}$ nominal pore size data was approximately $10 \mathrm{MPa}$ lower than the DMI curve for specimens with a porosity below 0.4 , but matched the curve very closely at higher porosities.

The measured yield strength values showed a significantly higher degree of scatter than the ultimate strength data, particularly at lower porosities. Specimens with similar porosity and nominal pore size could have a difference in yield strength of as much as $20 \mathrm{MPa}$. The measured data was consistently lower than the DMI curve, with the exception of $500 \mu \mathrm{m}$ nominal pore size specimens with a porosity above 0.4 . As with the ultimate tensile strength, these high porosity specimens closely matched the DMI data. The net area yield strength of the porous specimens was consistently higher than that of the nonporous material.

The nominal pore size of the specimens used in the DMI tests and the method of yield strength measurement are still unknown. The large amount of scatter in the measured data and the fact that most measured values are lower than the reported DMI values indicate that the four DMI data points may be the highest strength values obtained from a larger batch of specimens.

The results of the microscopy measurements and diffractometer testing done on the GASAR porous and nonporous specimens indicate that the following effects may influence the mechanical behavior:

- the ratio of grain size to specimen diameter

- the Hall-Petch effect of grain size 
- the preferred orientation of the material

- constraining effects due to triaxial or plane stress states

- the grain substructure

The impurity analysis performed on GASAR specimens has shown that the impurity content of the porous and nonporous specimens is very similar. The high purity of the material suggests that the impurities that are present do not have a significant effect on the mechanical properties.

\subsection{Effect of Grain Size vs. Specimen Size}

Because of the extremely large grains found in the nonporous GASAR specimens, the size of the tensile testing specimen may have a significant effect on the plastic behavior of the material. The ratio of specimen diameter to transverse grain size is 3.7, as compared to the ratio of 15 recommended by Hansen to negate any size effects. In contrast, this ratio varies between 8.4 and 26.7 in the porous GASAR specimens. Only the influence of this ratio on the ultimate tensile strength has not been adequately documented. Attempts to produce a nonporous specimen with a larger diameter without interference from the large diagonal pores were unsuccessful. Therefore, additional research is required to determine the exact influence of the specimen size on the mechanical behavior.

\subsection{The Hall-Petch Effect}

Figures 3.21 and 3.22 show that the grain size of GASAR materials is highly dependent on porosity. Thus the Hall-Petch effect must be considered with respect to the yield strength vs. porosity data. Because of the large amount of scatter in the yield strength data, the influence of the Hall-Petch effect on the porous GASAR yield strength is difficult to define. Considering that the nonporous specimens have a much larger grain size than any of the porous specimens, the Hall-Petch effect may have a strong influence on the net area yield strength difference between the nonporous and porous specimens. 
The Hall-Petch data from other sources indicate that a strength increase of up to $30 \%$ would be expected for the decrease in grain size observed in GASAR porous specimens. The magnitude of the strength difference between the porous and nonporous material is somewhat larger than this, meaning that Hall-Petch cannot completely explain this difference, but it could be a significant contributing factor.

Another discrepancy that the Hall-Petch effect may explain is the difference between the nonporous yield strength and the reference values for pure copper yield strength. Figure 3.28 illustrates that copper of high purity can have a yield strength significantly below the reference value. The GASAR materials are, in fact, very strong when compared to copper with a similar grain size.

\subsubsection{Influence of the Porous Grain Boundary on the Hall-Petch Effect}

Referring back to section 1.7.6, the operating mechanism of the Hall-Petch effect must be considered when applying it to GASAR porous materials. The Hall-Petch equation is based on a stress concentration due to dislocation pile-ups in one grain unlocking dislocations in an adjacent grain. A solid interface between the adjacent grains is assumed. Figure 3.23 shows that the grain boundaries in porous GASAR materials are approximately $50 \%$ free surface due to pores that nucleate on the boundary during solidification. These pores may significantly alter the influence of the Hall-Petch effect on material behavior. Another possible source of interference is pores contained within the grain. The magnitude of the stress concentration acting on adjacent grains is dependent on the pile up length, which is assumed to be the full diameter of the grain. Pores contained within a grain could change the effective length of dislocation pile-ups, thus decreasing the magnitude of the stress concentration. 


\subsubsection{Preferred Orientation and the Hall-Petch Effect}

Apart from the porous structure, an element of GASAR materials which may limit the influence of the Hall-Petch effect is the existence of strong preferred orientation. In polycrystalline materials with random orientations, the Hall-Petch effect manifests itself by slippage of the most favorably oriented crystals causing stress concentrations in lessfavorably oriented crystals. These stress concentration unlock dislocations in the grains and cause them to begin slipping. In GASAR materials, all grains are of similar $<100>$ orientation with respect to the testing direction, the only difference in orientation between adjacent grains being a rotation about the testing axis. This means that the slip planes of each grain are at roughly the same angle with regard to the applied stress. The exact effects of preferred orientation on the Hall-Petch effect are unknown.

\subsection{Preferred Orientation}

Apart from possible interference with the Hall-Petch effect, the preferred orientation of the GASAR materials has other significant implications. Both the physical and mechanical properties of single crystals are highly dependent on orientation. The high thermal conductivity in GASAR porous materials (see section 1.3.3) is most likely an effect of this orientation. The electrical conductivity will also be affected. The primary influences relevant to this study involve the elastic and plastic mechanical properties.

\subsubsection{Effects on Young's Modulus}

It is well established that the Young's modulus of a single crystal is highly dependent on the crystallographic direction in which it being strained. Because grain size does not effect the elastic behavior of a material and the elastic modulus does not involve slip, a polycrystalline material made of similarly oriented grains should have a modulus comparable to a single crystal of the same orientation. The minimum and maximum Young's moduli exist in the $<100>$ and $<111>$ directions, respectively. The modulus of 
copper crystals with an orientation of $<100>$ has been measured at from $60 \mathrm{GPa}-67$ $\mathrm{GPa}$, while <111> copper crystals have been measured at from $170 \mathrm{GPa}-190 \mathrm{GPa}{ }^{25,42}$. Randomly oriented polycrystalline copper has a modulus of approximately $110 \mathrm{GPa}^{4}$.

This dependence of the modulus on crystallographic orientation provides an explanation for the band of lower modulus values shown in Figure 3.11. The range of values measured for the modulus of copper in the $<100\rangle$ direction lies in the approximate center of this clustered band of data points. The upper band of modulus values between $107 \mathrm{GPa}$ and $120 \mathrm{GPa}$ are possibly due to the existence of grains of other orientations within the gage length of the tensile specimen. Although a very strong preferred orientation was evident in nearly all transverse cross-sections subjected to $\mathrm{x}$-ray diffraction, grains of other orientations were also indicated. The strength of the crystallographic texture varied from specimen to specimen. Due to the large size of the grains with respect to the specimen diameter and the length of the grains in the direction of testing, a small number of grains of $\langle 111\rangle$, or another high modulus orientation, could cause a significant increase in the modulus measurement for an individual specimen. In the case of the nonporous specimens, which showed comparable yield and ultimate tensile strengths but a large variation in modulus values, this seems to be a likely explanation, particularly since the nonporous material grains are so large with respect to the specimen diameter.

The mechanisms of elastic deformation used to develop the Gibson and Ashby model for the modulus of a closed cell foam could also have an effect on the measured modulus. These mechanisms may contribute to the large amount of scatter in the measured data.

\subsubsection{Effects on Yield Strength}

The influence of crystallographic texture on the measured yield strength of GASAR materials is much less straightforward. A numerical analysis based on the 
Taylor theory of plastic deformation gives a precise relationship between yield strength and orientation for polycrystalline FCC metals, as shown in Figure 4.1. Figure 4.1 gives the relationship between yield strength and texture in terms of the ratio of uniaxial yield strength to the critical resolved shear stress of the material. A number of theoretical studies have dealt with this relationship ${ }^{20,21}$, but there has been very little experimental verification of the influence of texture on the yield strength. Also to be considered is the fact that the derivation of this analysis ignores the Hall-Petch effect of grain size. Because the Hall-Petch effect is possibly significant in GASAR materials, it is difficult to isolate any possible effects of the orientation on yield strength. Comparison to data obtained for randomly oriented copper must be done with consideration of the relative grain size.

Figure 4.1 indicates that a material with $<100>$ preferred orientation should be

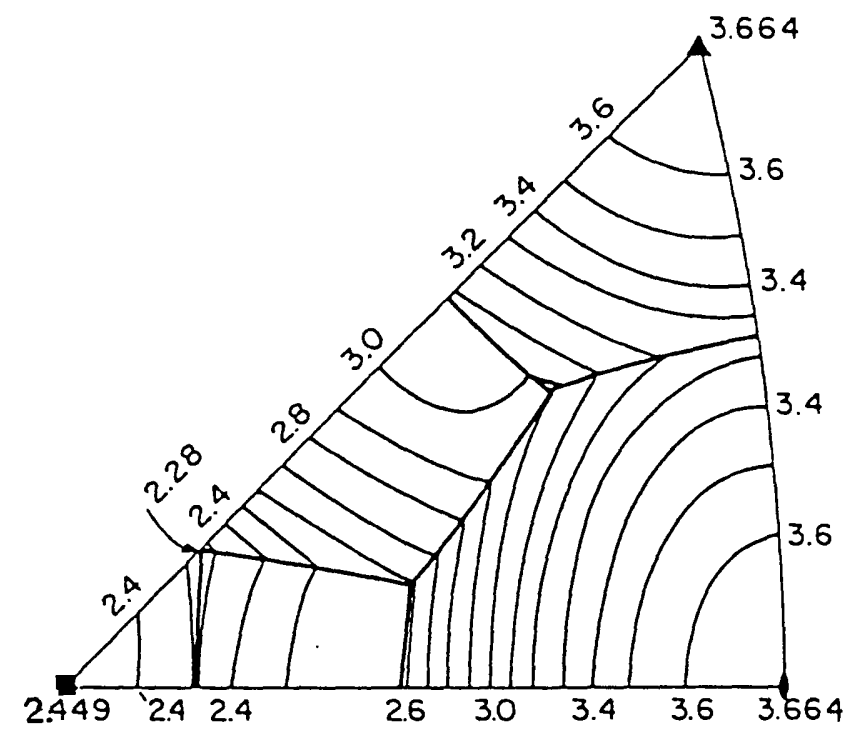

\section{Figure 4.1 - Polycrystalline Yield Strength vs. Texture}

The above diagram shows the ratio ' $m$ ' of polycrystal yield strength to the critical resolved shear stress required to yield a single crystal as a function of preferred orientation. (Taylor analysis) (Ref. 21) 
weaker than a randomly oriented polycrystal, but since the interaction between texture and grain size effects is undefined, it cannot be stated conclusively is this is an influencing factor in GASAR yield strength.

\subsection{Constraining Effects of the Porous Microstructure}

The decreasing ductility of the GASAR materials with increasing porosity indicates some sort of constraint being imparted on the solid material by the porous microstructure. The factors that have been discussed with respect to their possible influence on the elastic modulus and yield strength have a much smaller effect on material ductility. The ductility of the nonporous GASAR specimen is comparable with reference values of annealed copper of random crystallographic orientation and smaller grain sizes ${ }^{4}$.

It has been shown that the nonuniformity of the pore distribution can increase flow localization and lead to significant losses in ductility in elastic-plastic materials ${ }^{43}$. Because the nonuniformity of pore distribution in GASAR specimens is much higher at lower porosities, the reduction of ductility must be related to the increasing number of pores as well as their distribution. At fracture, the size of pores in the transverse crosssection are roughly the same as in the undeformed specimen. The pores consolidate within the cross section due to the necking of the solid material between the pores, but the pores themselves do not collapse or reduce in size. This indicates that the solid material of which the pore walls are composed is constrained in the direction circumferential to the pores. This constraint could be the result of a hoop stress caused by internal gas pressure or an effect of the plate-like shape of the pore wall. Because an extremely high gas pressure would be needed to have any significant effect, the latter explanation is more likely. Regardless, the existence of internal stresses in the normal plane of the uniaxial testing direction indicates a possible strengthening mechanism of the solid material. 


\subsubsection{Triaxial and Plane Stress States}

From the mechanisms discussed above, localized areas would be subject to biaxial and triaxial states of stress. The areas most likely to be affected are shown in Figure 4.2. Plane stress states will tend to occur in the walls separating adjacent pores, where the thickness of the section is much smaller than the width and length. Triaxial stress states may occur at points where a number of these walls converge. Figure 4.2 illustrates these areas on the transverse cross-section, but similar regions could also be found on the longitudinal cross-section. Further analysis is required to determine the extent of the influence these localized stress states could have on the net area yield strength.

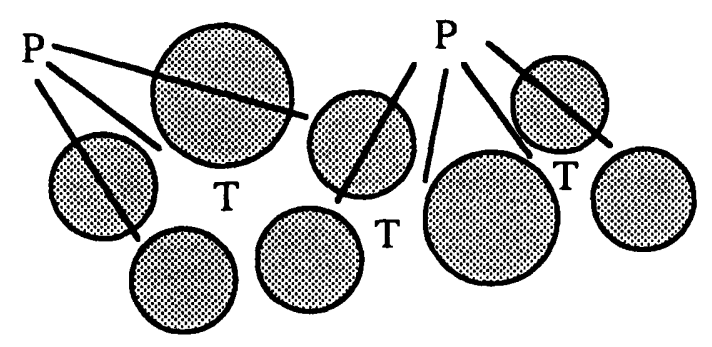

Figure 4.2 - Areas of Plane Stress and Triaxial Stress States

The above diagram indicates areas of possible plane stress (designated by "P") and triaxial stress ("T") states within the structure of the transverse cross-section of a GASAR porous specimen..

\subsection{Grain Substructure}

It was shown in section 3.6 that GASAR nonporous materials exhibit a grain substructure consisting of tilt boundaries running parallel to the longitudinal axis, as is consistent with a dendritic solidification front in unidirectional solidification. The level of substructure development in the nonporous material varied significantly from grain to grain. A similar substructure was not found in the grains of porous GASAR specimens. 
It has been shown that high pressure at solidification causes an increasingly fine substructure, such that the subgrains are smaller and more uniformly aligned ${ }^{42}$. Because of the small size of the substructure cells, it is difficult to ascertain whether the porous materials do not contain a substructure like that found in the nonporous samples, or the substructure is too fine to detect using the same etching procedure. The influence of subgrain boundaries and the level of grain substructure development on the mechanical properties of materials is not well defined, but since tilt boundaries consist of an organized structure of dislocations, which act as dislocation sources during deformation and impede the motion of other dislocations, it is reasonable to conclude that the substructure does affect the deformation behavior of a solid material.

\subsection{Summary}

The results presented in chapter three show that GASAR materials show an increase in the net area yield strength and net area tensile strength for a certain range of porosity values. It is also shown that this strength increase may be due, to a certain extent, to differences (grain size, substructure) between the solid material in the porous specimens and the nonporous material. GASAR materials can effectively be produced with oriented cylindrical pores with an aspect ratio of approximately 10 , but the consistency of the microstructure varies from specimen to specimen. 


\section{Chapter Five : Conclusions}

This chapter summarizes the conclusions that can be made from the experimental results presented in this thesis. Recommendations are also made for continuing research on GASAR materials.

\subsection{Conclusions}

A strengthening effect in the porous materials, similar to that reported by DMI, was found using tensile specimens with a reduced diameter of $5 \mathrm{~mm}$. It was also found, however, that this strengthening effect may be due to differences between the nonporous material and the solid cell wall material in the porous specimens. More information about the properties of the solid material in the nonporous and porous specimens is necessary to accurately apply the Gibson and Ashby equations to the properties of GASAR porous materials. Although a definitive explanation of the mechanical behavior of GASAR materials has not been developed, many conclusions can be made about the microstructure and behavior of the copper-based, longitudinal pore materials tested in this study.

The cylindrical pores are closed cell and have an average aspect ratio of approximately 10 . The aspect ratio of the pores is not highly dependent on the pore diameter. Although the average aspect ratio in each specimen was approximately the same, the distribution of pore diameters and aspect ratios varied significantly from specimen to specimen. All specimens contained pores that were highly unconnected and discontinuous. 
The directional solidification used in the GASAR process produces columnar grains with a high $\langle 100\rangle$ crystallographic texture in the solidification direction. Both the porous and nonporous GASAR specimens contained columnar grains with an aspect ratio of approximately 10. A $<100>$ texture was found in all specimens as well, although the degree of preferred orientation was higher in the porous specimens. The crystallographic texture has a strong influence on the Young's modulus of the material, and may have a significant effect on the yield strength as well.

The grain size of the nonporous material is significantly larger than that of the porous specimens. This difference may be significant in explaining the strengthening effect observed in the data obtained from $5 \mathrm{~mm}$ diameter waisted tensile specimens. The Hall-Petch effect indicates an increase in yield strength with the refinement of grain size. The difference in specimen size to grain size ratio in the nonporous and porous materials also indicates a strengthening effect with decreasing grain size.

The ductility of GASAR materials in the direction of pore orientation decreases with increasing porosity. The porous structure of GASAR materials localizes the plastic deformation of the solid cell wall material. The size of the pores in the transverse crosssection does not decrease during straining.

A subgrain structure of low angle boundaries exists in many columnar grains of the nonporous specimens. A well-defined substructure was found in many, but not all, nonporous specimen grains. A substructure was not found in the porous specimens, although it is possible that the etching method was too coarse for these samples.

\subsection{Recommendations for Future Research}

Several effects have been identified that may have a significant influence on the mechanical properties of GASAR materials. These effects include preferred orientation, the Hall-Petch effect, triaxial stress states, subgrain structure and the ratio of specimen size to grain size. Because these effects act simultaneously, efforts must be made to 
isolate the influence of each effect. Based on the results of this thesis, the following recommendations are made:

More $12.5 \mathrm{~mm}$ diameter tensile tests - The large size of the nonporous material grains in comparison to the $5 \mathrm{~mm}$ diameter specimen makes these tests very important. An increase in the nonporous material yield strength of a larger diameter specimen could completely, or partially, explain the strengthening effect observed in the $5 \mathrm{~mm}$ diameter specimens. Although the attempts made thus far to produce sound $12.5 \mathrm{~mm}$ diameter specimens have been unsuccessful, more attempts should be made. Because the nonporous materials are not dependent on a eutectic solidification process, studying the behavior of unidirectionally solidified materials obtained from other sources may be helpful.

Microhardness testing - Because the grains in the nonporous and porous materials are at a similar $<100>$ orientation, microhardness indentations made within individual grains on the transverse cross-section will give comparative data on the yield strength of the solid material. The microhardness is roughly proportional to the yield stress. The strain hardening quality of copper would make an exact determination of the yield strength questionable, but the relative magnitude of the yield stress could be determined as a function of porosity. Preliminary testing must be done to determine if accurate microhardness tests can be made in the proximity of pores, but a $5 \mathrm{~g}$ or $10 \mathrm{~g}$ load should yield unbiased results if the indentation is made far enough from the pore edges.

Finite element modeling - A finite element model of a solid material containing voids with a high aspect ratio could measure the influence of localized plane or triaxial stress states on the overall material properties. Such a model would eliminate the influence of grain size and orientation effects. The solid material of the model can be given an arbitrary yield strength and be governed by the von Mises yield criterion. Application of a uniaxial load to the model and measurement of the average stress in the 
direction of loading would indicate the influence of localized stress states on the material yield strength.

If the influence of individual effects on the material properties of GASAR materials can be defined, modifications can be made to the Gibson and Ashby models to allow them to accurately describe GASAR behavior. Future work should also include a study of the behavior of GASAR materials under compressive and transverse loads. 


\section{References}

1) D.M. Walukas, GASAR Materials: A Novel Approach in the Fabrication of Porous Metals, USP Holdings (unpublished report), 1992.

2) K. Russell, Modelling of GASAR Solidification, Massachusetts Institute of Technology (unpublished presentation made to the Office of Naval Research), 1994.

3) L.V. Boiko, V.I. Shapovalov, E.A. Chernykh, I.A. Belyi, I.A. Rastorgueva, V.P. Martynov, V.I. Dolzhenkov, Poroshkovaya Metallurgiya, No. 10 (346), pp. 78-81, 1991.(in Russian) Translated by Plenum Publishing Co., 1992.

4) Metals Handbook, 9th ed. v.2, Properties and Selection: Nonferrous Alloys and Pure Metals, American Society of Metals, 1979

5) L.J. Gibson, M.F. Ashby, Cellular Solids: Structure and Properties, Pergamon Press, 1988.

6) J.P. Holman, Heat Transfer, 5th ed. McGraw-Hill, 1981

7) G. Karam, Elastic Stability of Cylindrical Shells with Soft Elastic Cores: Biomimicking Natural Tubular Structures, Doctoral Thesis, Massachusetts Institute of Technology, 1994.

8) W.D. Callister, Jr. , Materials Science and Engineering: An Introduction, 2nd ed., John Wiley and Sons, 1991

9) E.P. Popov, Mechanics of Materials, 2nd ed., Prentice-Hall, Inc., 1976

10) L. Northcott, D.E. Thomas, Joural of the Institute of Metals, No. 65, pp. 205$216,1939$.

11) L.Northcott, Joural of the Institute of Metals, No. 69, pp. 189-207, 1942.

12) M.C. Flemings, Transactions of the American Foundryman's Society, V. 78, pp. 388-394, 1970.

13) A.F. Giamei, E.H. Kraft, F.D Lemkey, New Trends in Materials Processing, Conference Proceedings, American Society of Metals, 1974.

14) J.A. Eady, I.O. Smith, C. McL. Adam, Joural of the Institute of Metals, No. 101, pp. 162-166, 1973.

15) P. Haasen, Physical Metallurgy, Cambridge University Press, 1978.

16) C.S. Barret, T.B. Massalski, Structure of Metals, 3rd ed., McGraw-Hill Book Co., 1966. 
17) A. Suzuki, J. Nakamura, T. Sakamoto, Joural of the Japan Institute of Metals, V. 34, pp. 797-801, 1970 (in Japanese).

18) F.H. Wilson, R.M. Brick, Transactions AIME, V. 161, pp. 173-202, 1945.

19) M. Cook, T.Ll. Richards, Joural of the Institute of Metals, No. 69, pp. 201-207, 1943.

20) J.H. Laflen, Journal of Engineering Materials and Technology, V. 105, pp. 307$312,1983$.

21) W.F. Hosford, Jr., W.A. Bakofen, Strength snd Plasticity of Textured Metals, Ninth Sagamore Army Materials Conference on Fundamentals of Deformation Processing, pp. 259-293, 1962.

22) G.I. Taylor, Journal of the Institute of Metals, V. 62, pp.307-323, 1938.

23) J.J. Becker, J.N. Hobstetter, Transactions AIME, V. 197, p. 1231, 1953.

24) T.Ll. Richards, Progress in Metal Physics, pp. 281-305, ed. B. Chalmers, Interscience Pub., Inc., 1950.

25) J. Weerts, Zeitschrift Fur Metallkunde, V. 5, p. 25, 1933

26) F.D. Rosi, Transactions AIME, V. 200, pp. 1009-1020, 1954.

27) M.Z. Butt, Journal of Material Science Letters, V. 2, pp. 1-2, 1983.

28) P. Feltham, J.D. Meakin, Philosophical Magazine, V. 2, p. 105, 1957.

29) R.P. Carrecker, W.R. Hibbard, Acta Metallurgica, V.1, p. 654, 1953.

30) N. Hansen, Acta Metallurgica, V. 25, p. 863, 1977.

31) ASTM E 111, 1991 Annual Book of ASTM Standards, Sec. 3, V. 03.01, 1991.

32) ASTM E 8M, 1991 Annual Book of ASTM Standards, Sec. 3, V. 03.01, 1991.

33) E.O. Hall, Yield Point Phenomena in Metals and Alloys, Plenum Press, 1970.

34) Metallographic Sample Preparation, Metal Digest, V. 20 No. 3, Buehler, LTD., 1981.

35) A. Pattnaik, S.C. Sanday, H.I. Aaronson, Pore Size, Morphology, Distribution and Thermal Stability in $\mathrm{Cu}$ GASAR Ingots, Naval Research Laboratory, GeoCenters, Inc., (unpublished presentation made to the Office of Naval Research) 1994.

36) ASTM E 407, 1991 Annual Book of ASTM Standards, Sec. 3, V. 03.01, 1991.

37) ASTM E 340, 1991 Annual Book of ASTM Standards, Sec. 3, V. 03.01, 1991. 
38) M.A. Hayat, Principles and Techniques of Scanning Electron Microscopy Principles and Techniques of Scanning Electron Microscopy, V.1, Van Nostrand Reinhold Co., 1974.

39) ASTM E 112, 1991 Annual Book of ASTM Standards, Sec. 3, V. 03.01, 1991.

40) T. Gladman, F.B. Pickering, The Effect of Grain Size on the Mechanical Properties of Ferrous Metals, Yield, Flow and Fracture of Polycrystals, ed. T.N. Baker, Applied Science Publishers, 1983.

41) Analytical Report (untitled), submitted to Naval Research Laboratory by Luvak, Inc., Boylston, MA, 1994.

42) R.W. Hertzberg, Deformation and Fracture Mechanics of Engineering Materials, John Wiley and Sons, 1976.

43) J. W. Hutchinson, J. Mech. Phys. Solids, V. 32, No.1, pp. 63-85, 1984. 


\section{Biographical Note}

The author was born December 2nd, 1970 to Andrea and Anthony Simone, Sr. He was the second child of three, between Michelle and Nicole. He was raised in Wilmington, Delaware, where he graduated from Salesianum School for Boys in 1988. He attended Swarthmore College in Swarthmore, Pennsylvania, where he studied engineering, served as a resident assistant, and captained the football team. He graduated with distinction in 1992 with a Bachelor of Science in Engineering. His Senior Thesis implemented the AISC Load and Resistance Factor Design code in a macro code program package for the finite element analysis program, ANSYS. He was selected to Phi Beta Kappa, Tau Beta $\mathrm{Pi}$ and Sigma Xi. He entered the Department of Civil and Environmental Engineering at the Massachusetts Institute of Technology in 1992, where he will pursue a Ph.D.. He has passed the Engineer-In-Training exam, and is an active member of ASCE.

Previous publication:

F.M.A. Siddiqui, A.E. Simone, Jr., "An ANSYS Macro Program Package to Evaluate Steel Building Structures Using the 1986 AISC LRFD Specifications", 1994 ANSYS Conference Proceedings, Vol. 1, pp. 2.3 - 2.13, 1994. 